\title{
A CFD study of biomass pyrolysis in a downer reactor equipped with a novel gas-solid separator - I: hydrodynamic performance
}

\author{
Xi Yu ${ }^{1}$, Yassir Makkawi ${ }^{1 *}$, Raffaella Ocone ${ }^{2}$, Martin Huard ${ }^{3}$, Cedric Briens ${ }^{3}$ and \\ Franco Berruti ${ }^{3}$
}

1 European Bioenergy Research Institute (EBRI), School of Engineering and Applied Science, Aston University, Birmingham B4 7ET, United Kingdom

2 Chemical Engineering, Heriot-Watt University, Edinburgh EH14 4AS, UK

3 The Institute for Chemicals and Fuels from Alternative Resources (ICFAR), Western University, London, Canada

\section{Abstract:}

This study presents the first part of a CFD study on the performance of a downer reactor for biomass pyrolysis. The reactor was equipped with a novel gas-solid separation method, developed by the co-authors from the ICFAR (Canada). The separator, which was designed to allow for fast separation of clean pyrolysis gas, consisted of a cone deflector and a gas exit pipe installed inside the downer reactor. A multi-fluid model (Eulerian-Eulerian) with constitutive relations adopted from the kinetic theory of granular flow was used to simulate the multiphase flow. The effects of the various parameters including operation conditions, separator geometry and particle properties on the overall hydrodynamics and separation efficiency were investigated. The model prediction of the separator efficiency was compared with experimental measurements. The results revealed distinct hydrodynamic features around the cone separator, allowing for up to $100 \%$ separation efficiency. The developed model provided a platform for the second part of the study, where the biomass pyrolysis is simulated and the product quality as a function of operating

\footnotetext{
* Corresponding author. +44 (0)121 204 3398; email: y.makkawi@aston.ac.uk
} 
conditions is analysed.

Key words: Biomass pyrolysis, downer reactor, gas-solid separation, CFD modeling, hydrodynamics

\section{Introduction}

The escalating global concern over the exhaustion of non-renewable energy sources lead to the recent development of a range of novel technologies for the use of renewable energy resources, such as biomass, solar and wind. Among these resources and technologies, biomass pyrolysis has emerged as a very promising renewable alternative for bio-oil production. In a large commercial scale, this could be carried out in a dual fluidized bed (DFB) system with various optional arrangements. The schematics in Fig. 1 demonstrate examples of these arrangements. In this study, we are interested in the downer-riser type of a dual fluidized bed, shown in Fig. 1-b, where the biomass pyrolysis takes place in the downer side of the reactor, while the riser side is used for combustion, thus providing the heat required for the pyrolysis through the circulating inert heat carrier solid (such as sand). This arrangement has the following specific advantages for bio-oil production through fast pyrolysis:

i. The downer pyrolysis reactor can be operated with very low carrier gas (e.g. nitrogen) flow rates, which is desirable in some cases to reduce up-stream pre-heating and downstream processing.

ii. Reducing the gas and solid back-mixing [1, 2, 3] thus, limiting the spread of the gas/solid residence time distribution, i.e. near to plug flow.

iii. Relatively low cost, simple operation/control and high energy efficiency.

iv. The char combustion in the second reactor will guarantee sustainable operation and better control of the pyrolysis temperature in the first reactor.

However, in order to achieve high conversion efficiency (more than $70 \%$ bio-oil yield) in a downer reactor there remains two main technical challenges: 
i. Control of the pyrolysis gas residence time within the hot zone of the reactor (ideally 1-2 seconds). Longer residence time of the pyrolysis gas at high temperature initiates a range of undesirable side reactions, which could adversely affect the quality of the product bio-oil $[4,5]$

ii. Control of the downstream contact between the pyrolysis gas and bio-char. The bio-char, formed during pyrolysis, acts as a vapor cracking catalyst, therefore should be separated as soon as the pyrolysis vapor is released [6]

Char, as well as other entrained fine particles, can primarily be separated from the pyrolysis gas by using conventional cyclones (reverse and co-current flow types). However, this carries the risk of increasing the contact time between the gas and char inside the cyclone. In addition, the cyclone inlet is commonly placed external to the reactor or away from the pyrolysis zone, thus, causing extra contact time between the solid and gas. The extensive review conducted by Huard et al. [7] and Cheng et al. [8] on downer reactors and rapid gas-solid separation techniques revealed that there are limited attempts on implementing new design methods for rapid gas-solid separation in these reactors.

Recent research at the Institute for Chemicals and Fuels from Alternative Resources (ICFAR) has led to the development of a novel gas-solid separation device for a downer pyrolysis reactor. The device features a cone-shaped solid deflector positioned above a gas outlet pipe, both positioned concentrically in the downer pipe (see Fig. 2). This was designed to achieve primary solid-gas separation and gas removal within the same device [9] The separator allows for better control of the pyrolysis vapor residence time, therefore, reducing the severity of vapor overcracking compared to other fast separation methods. Experimental work by Huard et al. [10] has shown that this separator can achieve very high solid-gas separator efficiency above $99.99 \%$ when using spherical silica sand particles of $200 \mu \mathrm{m}$ 
diameter.

Computational Fluid Dynamics (CFD) modeling is one of the powerful tools to analyse gas-solid flow behavior, including that involves intense heat transfer and chemical reactions. The co-authors from the ICFAR have previously used an Eulerian-Lagrangian modeling approach to investigate the effect of the particle elasticity on the separator efficiency in the same novel separator investigated in this study [9]. While this approach revealed important details of the particle-wall collision and its effects on the separator efficiency and mechanism, the simulation domain was limited to the separator zone only and the total solid volume fraction was limited to a maximum of $4 \times 10^{-5}$. The Eulerian-Eulerian (also referred to as two-fluid) is another modeling approach that has the advantage of being robust and realistic in computational time, especially when considering a large number of particles or large simulation domain. Unlike the Eulerian-Lagrangian approach, which treats each single particle as a dispersed phase in the continuum fluid flow, the Eulerian-Eulerian approach treats both of the fluid and solid phases as an interpenetrating continuum. Studies on the Eulerian-Eulerian simulation of solid-gas hydrodynamics in a downer reactor have been previously reported by Ropelato et al. [11], Kim et al [12] and Samruamphianskun [13] This modeling approach was found to be especially useful in predicting the effects of inlet design and flow conditions on the solid distribution and dispersion behavior. This CFD modeling approach has also been used by different researchers to study the phenomena of solid-gas separation in cyclones $[14,15,16]$

In this study, the main objectives are:

i. to develop a valid Eulerian-Eulerian (multi-fluid) CFD model capably of predicting the detailed hydrodynamic behaviour in a downer reactor equipped with a novel gas-solid separation device; 
ii. to use the developed model in investigating the effect of the operating conditions and various separator design parameters on the overall hydrodynamics, with particular focus on the separator efficiency;

iii. to provide a platform for the development of a predictive model of the pyrolysis reactions and yield in the downer reactor equipped with the novel gas-solid separator.

The investigation was carried out theoretically and experimentally in a cold flow reactor model equipped with the ICFAR novel gas-solid separator and gas removal mechanism, as described in details in the experimental section. The theoretical transient model was solved in three-dimensional coordinates using the EulerianEulerian (two-fluid) approach, employing constitutive relations from the kinetic theory of granular flow (KTGF) [17]. In the second part of this study, the developed hydrodynamic model will be extended to include heat transfer and reaction kinetics to demonstrate the advantages of the ICFAR separator in improving the performance and product quality in a biomass downer pyrolysis reactor.

\section{Experiments and procedure}

The experimental work described here was carried out by the co-investigators at the ICFAR in Canada. The equipment consisted of a cold flow gas-solid flow downer of $133.5 \mathrm{~cm}$ height and $7.0 \mathrm{~cm}$ diameter, equipped with the ICFAR novel gas-solid separator as shown in Fig. 2 and 3. This separator included a gas removal pipe and a cone deflector, where the bottom of the deflector and tip of the pipe were located $98.6 \mathrm{~cm}$ below the downer inlet. A solid collection tank of $20.4 \mathrm{~cm}$ diameter and 21.8 $\mathrm{cm}$ height was placed at the bottom of the downer column around $34.9 \mathrm{~cm}$ below the cone deflector. Compressed air at room temperature was supplied to the downer from a bank of calibrated sonic orifice nozzles. The Sauter mean diameter of the particle mixture used was $188 \mu \mathrm{m}$ and its skeletal density was $2650 \mathrm{~kg} / \mathrm{m}^{3}$. The 
particle size distribution of the mixture is shown in Fig. 4.

The solid particles were delivered to the downer column from an air pressurized tank mounted above the downer main air inlet. The total mass flowrate of air in the downer was $\dot{m}_{g}=0.0039 \mathrm{~kg} / \mathrm{s}$, which corresponded to a superficial gas velocity of $U_{g}$ $=0.73 \mathrm{~m} / \mathrm{s}$. The solids mass flowrate was adjusted by changing the feed tank air pressure and this was varied between $\dot{m}_{s}=0.017 \mathrm{~kg} / \mathrm{s}$ and $0.083 \mathrm{~kg} / \mathrm{s}$, which corresponded to solids-to-gas loading ratios of $\dot{m}_{s} / \dot{m}_{g}=4.3$ to 21 . The gas-solids mixture flowed co-currently in the downer before entering the gas-solids separation zone. Three different cone deflectors with various internal angles of $60^{\circ}, 90^{\circ}$ and $120^{\circ}$ were used. The downward falling particles were collected in the tank at the bottom of the unit, while the gas stream, along with any entrained particles, exited the system through the gas outlet pipe mounted in the centre of the downer cross section and below the cone deflector. A bag filter connected at the end of the gas exhaust line was used to collect the particles entrained in the exiting gas stream.

At the start of each experiment, the total mass of solids fed into the system, $m_{i n}$, was measured. The mass of the entrained solids collected in the bag filter, $m_{\text {collected, }}$ was then measured at the end of each experiment. Thus, the experimental percentage total solids separation efficiency, $\eta$, was calculated from the following expression:

$\eta=\left(1-\frac{m_{\text {collected }}}{m_{\text {in }}}\right) \times 100 \%$

The mass flowrate of solids was determined by measuring the total mass of solid collected in the filter bag and tank against the recorded time. 


\section{Hydrodynamic model}

The overall reactor hydrodynamics and gas-solid separation was investigated using the Eulerian-Eulerian (multi-fluid) model approach based on the Kinetic Theory of Granular Flow (KTGF). The developed model was solved using the CFD software ANSYS FLUENT (Ver. 14). In order to mimic the wide size distribution of the solid mixture used in the experiment, the simulation was carried out using a solid mixture of three different particle sizes, as detailed in section 3.3. The main model equations for non-reacting isothermal gas-solid flow are given by:

Continuity equations:

$\frac{\partial\left(\alpha_{g} \rho_{g}\right)}{\partial t}+\nabla\left(\alpha_{g} \rho_{g} \vec{u}_{g}\right)=0$

$\frac{\partial\left(\alpha_{s_{i}} \rho_{s}\right)}{\partial t}+\nabla\left(\alpha_{s_{i}} \rho_{s} \vec{u}_{s_{i}}\right)=0$

$\sum_{i=1}^{3} \alpha_{s_{i}}+\alpha_{g}=1$

Momentum equations:

$$
\begin{aligned}
& \frac{\partial\left(\alpha_{g} \rho_{g} \vec{u}_{g}\right)}{\partial t}+\nabla\left(\alpha_{g} \rho_{g} \vec{u}_{g} \vec{u}_{g}\right)=-\alpha_{g} \nabla P+\nabla \overline{\bar{\tau}}_{g}-\sum_{i=1}^{3} \beta_{g s_{i}}\left(\vec{u}_{g}-\vec{u}_{s_{i}}\right)+\alpha_{g} \rho_{g} g \\
& \frac{\partial\left(\alpha_{s_{i}} \rho_{s} \vec{u}_{s_{i}}\right)}{\partial t}+\nabla\left(\alpha_{s_{i}} \rho_{s} \vec{u}_{s_{i}} \vec{u}_{s_{i}}\right)=-\alpha_{s_{i}} \nabla P-\nabla P_{s_{i}}+\nabla \overline{\bar{\tau}}_{s_{i}}+\beta_{g s_{i}}\left(\vec{u}_{g}-\vec{u}_{s_{i}}\right) \\
& +\sum_{j=1, j \neq i}^{3} \beta_{s_{i} s_{j}}\left(\vec{u}_{s_{j}}-\vec{u}_{s_{i}}\right)+\alpha_{s_{i}} \rho_{s} g
\end{aligned}
$$

where:

$$
\begin{aligned}
& \overline{\bar{\tau}}_{k}=\left(\lambda_{k}-\frac{2}{3} \mu_{k}\right)\left(\nabla \cdot \vec{u}_{k}\right) \overline{\bar{I}}+2 \mu_{k} \overline{\bar{S}}_{k} \\
& \overline{\bar{S}}_{k}=\frac{1}{2}\left(\nabla \vec{u}_{k}+\left(\nabla \vec{u}_{k}\right)^{T}\right)
\end{aligned}
$$

$k$ represents solid or gas phase.

To obtain the granular temperature, the FLUENT code was optionally set to use a partial differential equation (Pseudo Energy Equation) as follows [18]: 


$$
\begin{aligned}
\frac{3}{2}\left[\frac{\partial\left(\alpha_{s_{i}} \rho_{s} \Theta_{s_{i}}\right)}{\partial t}\right. & \left.+\nabla\left(\alpha_{s_{i}} \rho_{s} \Theta_{s_{i}}\right) \vec{u}_{s_{i}}\right] \\
& =\left(-P_{s_{i}} \overline{\bar{I}}+\overline{\bar{\tau}}_{s_{i}}\right): \nabla \vec{u}_{s_{i}}+\nabla\left(\kappa_{T} \nabla \Theta_{s_{i}}\right)-\gamma_{T}+\sum_{k=1}^{3} \phi_{k s_{i}}
\end{aligned}
$$

The various closure and constitutive relations used in the model are given in Table 2. In order to take into consideration the solid-solid frictional stresses at the dense regions of the reactor, the friction equation proposed by Schaeffer [19], as given in Equation T1-5, was used. Due to the highly turbulence of the flow near the deflector zone the standard K-epsilon turbulence and energy dissipation equations proposed by Launder and Spalding [20] were also incorporated in the model and these are given as follows:

Turbulence momentum equations:

$\frac{\partial\left(\alpha_{g} \rho_{g} k_{g}\right)}{\partial t}+\nabla\left(\alpha_{g} \rho_{g} \vec{u}_{g} k_{g}\right)=\alpha_{g} G_{k, g}+\nabla\left(\alpha_{g} \frac{\mu_{t, g}}{\sigma_{k}} k_{g}\right)-\alpha_{g} \rho_{g} \varepsilon_{g}+\alpha_{g} \rho_{g} \Pi_{k, g}$

Turbulent kinetic energy dissipation equation:

$\frac{\partial\left(\alpha_{g} \rho_{g} \varepsilon_{g}\right)}{\partial t}+\nabla\left(\alpha_{g} \rho_{g} \vec{u}_{g} \varepsilon_{g}\right)=\nabla\left(\alpha_{g} \frac{\mu_{t, g}}{\sigma_{\varepsilon}} \varepsilon_{g}\right)+\alpha_{g} \frac{\varepsilon_{g}}{k_{g}}\left(C_{1 \varepsilon} G_{k, g}-C_{2 \varepsilon} \rho_{g} \varepsilon_{g}\right)+\alpha_{g} \rho_{g} \Pi_{\varepsilon, g}$

where:

$$
\begin{aligned}
& G_{k, g}=\mu_{t, g}\left(\nabla \vec{u}_{g}+\left(\nabla \vec{u}_{g}\right)^{T}\right): \nabla \vec{u}_{g} \\
& C_{\mu}=0.09, C_{1 \varepsilon}=1.44, C_{2 \varepsilon}=1.92, \sigma_{k}=1, \sigma_{\varepsilon}=1.3
\end{aligned}
$$

\subsection{Computational domain and meshing}

Fig. 5 shows the computational domain and the meshing used in solving the model. This was generated using a finite volume method with hybrid cells of structured and unstructured grids, giving a total of 30,785 cells. In order to capture the steep hydrodynamic variations around the walls of the separation device (the conical deflector and the gas exit pipe), the grid size was refined by setting the minimum and 
maximum grid size at 0.3 and $1.0 \mathrm{~cm}$ respectively. In the rest of the simulation domain the minimum and maximum grid size was set at 1.0 and $5.0 \mathrm{~cm}$ respectively. The impact of the grid size on the solution accuracy was initially tested by setting three different meshing schemes and the grid size used in this study was found to give acceptable grid independent solution.

\subsection{Computation procedure}

The model equations were solved using the finite volume approach. First-order discretization schemes were used for the solution of the convection terms in all governing equations. The relative error between any two successive iterations was specified by using a convergence criterion of $10^{-3}$ for each scaled residual component. The phase-coupled SIMPLE (PC-SIMPLE) algorithm [21], which is an extension of the SIMPLE algorithm to multiphase flows, was applied for the pressure-velocity coupling. The linearized equations for governing equations were solved using a block algebraic multigrid method. In order to ensure easy convergence of the various partial differential equations (PDE) in the model, the Courant-Friedrichs-Lewy (CFL) condition for three-dimensional PDE is followed:

$$
\mathrm{C}=\frac{\mathrm{u}_{\mathrm{x}} \Delta \mathrm{t}}{\Delta \mathrm{x}}+\frac{\mathrm{u}_{\mathrm{y}} \Delta \mathrm{t}}{\Delta \mathrm{y}}+\frac{\mathrm{u}_{\mathrm{z}} \Delta \mathrm{t}}{\Delta \mathrm{z}} \leq \mathrm{C}_{\max }
$$

where $C_{\max }$ is specified by the CFL condition to fall within the range of $\sim 1-5$ [22]. In this study, a time step of 0.005 seconds was found to satisfy this condition.

\subsection{Boundary and simulation conditions}

The particle-wall restitution coefficient and the specularity coefficient are two important parameters in determining the dynamics of particles at the wall region. The following wall boundary conditions were employed in the model [23]:

$$
u_{s_{i}, w}=-\frac{6 \mu_{s_{i}} \alpha_{s, \max }}{\sqrt{3 \Theta_{s_{i}}} \pi \varphi \rho_{s} \alpha_{s_{i}} g_{0, s_{i} s_{i}}} \frac{\delta u_{s_{i}, w}}{\delta n}
$$


$\Theta_{s_{i}}=-\frac{k_{s_{i}} \Theta_{s_{i}}}{\gamma_{w}} \frac{\delta \Theta_{s_{i}, w}}{\delta n}+\frac{\sqrt{3} \pi \varphi \rho_{s} \alpha_{s_{i}} u_{s_{i}, s l i p}^{2} g_{0, s_{i} s_{i}} \Theta_{s_{i}}{ }^{\frac{3}{2}}}{6 \alpha_{s, \max } \gamma_{w}}$

$\gamma_{w}=\frac{\sqrt{3} \pi\left(1-e_{s_{i}, w}^{2}\right) \rho_{s} \alpha_{s_{i}} g_{0, s_{i} s_{i}} \Theta_{S_{i}}{ }^{\frac{3}{2}}}{4 \alpha_{s, \max }}$

where $\varphi$ is the specularity coefficient and $e_{s_{i}, w}$ is the particle-wall restitution coefficient. In order to reasonably match the particle size distribution used in the experiments, the simulations were carried out assuming the solid mixture to consist of three different particle sizes. The fraction of each particle size group was estimated from the experimental size distribution given in Fig. 4. The simulation particle sizes and percentages are given in Table 3. For the gas phase, the velocity at the wall was assumed zero (no slip condition). Table 4 summarizes the various operating conditions considered in the simulations. Some of these conditions were carefully selected to allow for the comparison of the model predictions with the corresponding experimental data.

\section{Results and discussion}

\subsection{Mechanism of gas-solid separation}

It is postulated that the drag and gravity forces, the last two terms in the left hand side of Eq. 3b, are the main forces dominating the hydrodynamic behavior of the gas-solid phases within the separator zone. Fig. 6 gives an overall description of the flow structure with close zoom-in at the cone deflector region. The gas velocity in the gap between the deflector and the wall is very high due to the considerable pressure drop, similar to gas expansion through a throttling device. In the region under the cone and below the gas exit pipe there is an upward gas drag force due to the high reverse gas phase velocity. However, the extremely dilute solid concentration in this zone means very little solids are being entrained. It is therefore desirable to minimize the upward gas drag force in this region in order to achieve high separator efficiency. On top of the cone deflector, the solid phase is diverted radially towards the wall and 
then accelerates through the gap between the deflector and the downer wall, pushed by a strong gas drag force. The influence of the gravity force in this region is also significant due to the high solid concentration. Accordingly, it is believed that any particles entrained through the exit pipe are falling under the influence of two different drag mechanisms:

i. Reverse gas flow (upward) under the cone deflector due to the abrupt gas pressure drop at the tip of the gas exit pipe. This makes the tip of the exit pipe act as a vacuum to the surrounding solids.

ii. Radial gas flow from the walls towards the core in the region just below the cone deflector. This results in the solids being first dragged towards the core, and then further dragged/sucked by the gas leaving through the exit pipe.

This is to some extent similar to the solid-gas separation mechanism in a cyclone, where in both cases the reverse gas flow in the core is responsible of solid entrainment. However, in the cyclone, the particles move radially towards the walls under the influence of centrifugal forces, while in the cone deflector, the particles are deflected radially by the cone wall to fall under the strong downward gas drag force in the "throttling" gap, as described earlier. It is worth noting that the modeling results reported by the co-authors from the ICFAR suggested that the solid rebound upon hitting the walls, investigated through changing the wall-particle restitution coefficient, may have a dominant role in the mechanism of gas-solid separation in the cone deflector. This hypothesis will be discussed in some details in the following sections.

Fig. 7 shows the predicted solid concentration and the gas velocity profiles at the level of $3.9 \mathrm{~cm}$ below the tip of the gas exit pipe (see Fig. $6 \mathrm{~b}$ for the sampling line level). These profiles reveals a very interesting hydrodynamic behavior where the solid concentration profile is shown to take the shape of a dense-wall and dilute- 
core, while the gas velocity takes the shape of an upward parabolic flow profile at the core and a downward flow at the walls. It appears that, due to the existence of the cone deflector, the overall flow pattern below this device has been completely changed from the classic gas-solid down flow pattern, commonly observed in downer reactors, to a more complex flow similar to that existing in a turbulent solid-gas flow riser.

\subsection{Gas disengagement height (GDH)}

Fig. 8 shows that there are four distinct flow zones each with characteristic flow behavior. These are mainly arising from the changes induced by the cone deflector and these can be described as flows:

Zone I: This is where fully developed flow and uniform distribution of the solid and gas phases take place, typical to that observed in a conventional downer reactor.

Zone II: This the where both of the solid and gas phases are first hitting the inclined plane to create a dense moving solid layer at the cone walls before being pushing by a strong gas drag force through the gap between deflector and the downer walls. Under the cone, the lowest solid concentration in the whole system exists and the gas is removed through the exit pipe driven by the rapid pressure drop at the exit pipe tip.

Zone III: This is where the disengagement of gas from the gas-solid flow mixture takes place. The overall flow hydrodynamics in this region is very complex due to the effect of sharp changes in pressure, which consequently leads to reverse gas flow towards the top and radial solid movement from the dense walls towards the dilute core.

Zone IV: This is where the solid phase is mainly concentrated at the walls. The radial flow diminishes and the particles fall under the strong influence of the gravity force before entering the solid collection tank at the bottom of the downer system. 
As described earlier, the main objective of the cone separator is to allow for fast and efficient separation of the gas from the downward gas-solid flow stream. In a biomass pyrolysis downer reactor, this separation should ideally take place immediately at the level of the gas outlet pipe tip and with zero solid entrainment. The first reason is to prevent undesirable secondary gas reactions by removing the gas from the reactor hot zone, and second, to prevent catalytic char cracking by limiting the contacts between the solid and gas. However, in reality, the gas separation from the solid-gas stream takes place a little further down beyond the level of the tip of the gas removal pipe. It is therefore particularly interesting to quantify the height of Zone III, in which the gas separation takes place. This is defined here as the gas disengagement height (GDH), analogous to the definition of the transport disengaging height (TDH) in gas-solid fluidized beds. The method used in this study to estimate the GDH is demonstrated in Fig. 9. The GDH is defined at the intercept of the lines tangential to the low pressure gradient curve and the steep changing pressure gradient curve, or alternatively, the GDH can be estimated from plotting the axial gas velocity against height as shown in Fig. 9-b. The pressure gradient method is similar to the method used by Geldart et al. [24] in determining the transport disengaging height $(\mathrm{TDH})$.

Fig. 10 shows the result of a sensitivity analysis of the GDH to a range of operating conditions. Please note that the y-axis in Fig. 10 represents the summation of the the $\mathrm{GDH}$ and the separation distance $L_{s}$, where the separation distance is defined as the distance from the cone rim to the tip of the gas exit pipe. The GDH range under the various operation conditions considered in the simulation was found to fall between $2.5 \mathrm{~cm}$ and $6 \mathrm{~cm}$. It is clear that the $\mathrm{GDH}$ is most sensitive to the solid loading and the solid flow rate. The increase in the separation distance from $0 \mathrm{~cm}$ to $7 \mathrm{~cm}$ and the solid flow rate from $0.004 \mathrm{~kg} / \mathrm{s}$ to $0.08 \mathrm{~kg} / \mathrm{s}$ caused a corresponding increase in GDH of around $30 \%$ for both cases. Clearly, the cone angle and gas flow rate appear 
to cause negligible effect in this regard. It should be noted that, while it is desirable to decrease the GDH as discussed earlier, this does not necessarily mean improving the separation efficiency, as will be demonstrated in the next section.

\subsection{Separator efficiency}

The theoretical separator efficiency was obtained by dividing the predicted solid flow rate at the gas exit pipe (entrained solids) by the inlet solid mass flow rate, such that,

$\eta=\frac{\dot{m}_{s, \text { exit }}}{\dot{m}_{s, i n}} \times 100 \%$

The separator efficiency was analysed with respect to various operating conditions. This also included a sensitivity analysis of the separation efficiency towards varying the wall-particle interactions mechanism, through changing the particle-wall restitution coefficient and specularity coefficient. Both parameters appear in the solid boundary condition of Eq. 8-9. The first coefficient is a measure of the degree of energy loss when the particles hit the walls, hence determining the rebound velocity, and the second coefficient defines the angle of rebound. It is therefore possible to determine the effect of the wall surface material and particle properties on the separation efficiency through changing these two parameters in the model.

In this study, $100 \%$ separator efficiency was obtained when operating with: large particle size of $d_{p}=328 \mu \mathrm{m}$, separation length $L_{s}=0 \mathrm{~cm}$, cone angle $\theta=60^{\circ}$, gas mass flow rate $m_{g}=0.0039 \mathrm{~kg} / \mathrm{s}$ and high solid flow rate $m_{s}=0.08 \mathrm{~kg} / \mathrm{s}$. This was found to dramatically decrease when decreasing the particle size. This result is in good agreement with the experimental study by Huard et al [10] where it was shown that the separator efficiency, when using FCC catalyst of $d_{p}=43 \mu \mathrm{m}$ and glass beads of $d_{p}=63 \mu \mathrm{m}$, is much lower than that achieved with sand of $d_{p}=200 \mu \mathrm{m}$. Fig. 11 shows the values of the predicted separator efficiency obtained within the range of operating conditions considered in this study. The detailed results and 
discussion on the effect of each of these parameters on the overall hydrodynamics and separator efficiency are given in the next sections. According to this data, it is concluded that the sensitivity of the separator efficiency towards the operating conditions can be ranked in order of decreasing impact on the separation efficiency as follows; (1) separation length (2) cone angle (3) gas flow rate (4) solid flow rate (5) particle physical properties (expressed in terms of the restitution and specularity coefficients). Note that, the impact of particle size on the separation efficiency comes on top of all the above parameters.

\subsubsection{Effect of the separation length $\left(L_{s}\right)$}

The effect of separation distance on the separator efficiency was studied using three different separation lengths of $0 \mathrm{~cm}, 3.5 \mathrm{~cm}$ and $7 \mathrm{~cm}$, which corresponds to the normalized separation length $\left(L_{s} / D\right)$ of $0,0.5$ and 1 respectively. All the other parameters were set at the default values.

Figure 12 shows the separator efficiency as a function of the normalized separation length. The overall trend indicates a negative impact on the separator efficiency. The maximum mean efficiency was $99.986 \%$ and this dropped to $99.633 \%$ at $L_{s} / D=1$. The greater separation efficiency achieved with the particle size of $206 \mu \mathrm{m}$ compared to the size group of $324 \mu \mathrm{m}$ can be explained by the fact that the concentration of this particle group (60 wt\%) was greater than the latter one (20 wt\%). Therefore, the more frequent particle-particle interaction within the same group can neutralized part of radial velocity which may cause entrainment of particle. The same phenomenon was observed in Fig 15. In terms of sensitivity, the effect of the separation length on the separation efficiency is the highest compared to the other parameters investigated, as shown earlier in Fig. 11. It was also demonstrated earlier that the separation distance has also a relatively high effect on the GDH. The velocity vectors shown in Fig. 13 indicate that the increase in $L_{s}$ resulted in the creation of two vortices in the space between the cone deflector and the tip of the gas exit pipe. This 
can be attributed to the strong radial gas flow in this region, resulting from the considerable pressure drop at the exit pipe.

Fig. 14 shows the changes in the solid concentration and velocity profiles with changing the separation length at the sample level of $3.9 \mathrm{~cm}$ below the bottom of the cone deflector. It is clear that the solid concentration increases with increasing $L_{s}$, while the vertical upward gas velocity at the core decreases. This suggests that the upward gas drag force may have limited influence on the separation efficiency. It is the increased radial gas velocity (radial drag), the subsequent formation of vortices and the increased solids concentration at the core that collectively play the dominant role in decreasing the separator efficiency as the separation length increases.

\subsubsection{Effect of the cone deflector angle $(\theta)$}

The effect of the cone deflector angle on the separator efficiency was studied using various angles $\theta=60^{\circ}, 90^{\circ}$ and $120^{\circ}$. All other operating conditions were set at the default values. Fig. 15 shows that the separator efficiency decreases with increasing the cone angle. The maximum mean separator efficiency (taking into account the three particle sizes) was $99.986 \%$, this dropped to $99.869 \%$ efficiency when the angle is increased to $120^{\circ}$. This trend is in satisfactory agreement with the experimental data of Huard et al. [10]; however, the experiments showed less pronounced changes compared to the predictions, and this may be attributed to the differences between the particle size distribution in the experiment and the assumed size mixture in the model.

In Fig. 16 the magnitude and direction of the gas velocity vectors suggest that as the cone angle increases there is a greater chance the particles rebound more in the reverse direction from the cone inner surface and normal to the gas exit. This would slow down the particles and make them easier to be entrained, thus having a negative impact on the separator efficiency. In Fig. 17 there is clear increase in the 
solid concentration on top of the cone's upper surface due to flattering of the cone external surface as shown in Fig. 17; however this is not expected to have contributed to the change in the separation mechanism or efficiency.

\subsubsection{Effect of the gas mass flow rate}

The effect of inlet gas mass flow rate on the gas separator efficiency was investigated at three different flow rates of $0.0039 \mathrm{~kg} / \mathrm{s}, 0.0239 \mathrm{~kg} / \mathrm{s}$ and $0.0439 \mathrm{~kg} / \mathrm{s}$ and a fixed solid flow rate of $0.004 \mathrm{~kg} / \mathrm{s}$. This corresponds to inlet gas velocities of $0.73 \mathrm{~m} / \mathrm{s}, 4.5 \mathrm{~m} / \mathrm{s}$ and $8.2 \mathrm{~m} / \mathrm{s}$, respectively. All other operating conditions were set to the default values. Fig. 18 shows that the effect of the gas mass flow rate on the separator efficiency is negligible. This is in good agreement with the experimental observation reported by Huard et al. [10].

To gain further understanding on the effect of gas flow rate on the overall hydrodynamics, Fig. 19 shows the gas velocity vectors as function of the gas mass flow rate. It is clear that there is a significant change in the magnitude of the gas velocity but little change in the flow pattern. There is also evidence of a significant change in the solid concentration around the cone deflector as shown in Fig. 20. Despite this, such a dramatic change caused no effect on the separator efficiency due to counterbalance of forces, which are described as follows:

i. At a high gas velocity, there is considerable increase in the pressure drop between the gas exit pipe and its surroundings, hence high upward gas velocity (drag force), as shown in Fig. 21a. However, this is counterbalanced by the considerable reduction in the solid concentration in the wall and the core region below the exit pipe, as shown in Fig. 21b.

ii. At a low gas velocity, there is high solid concentration at the wall (i.e. high gravity force), as shown in Fig. 21b. This is associated with low pressure drop between the wall and the tip of the exit pipe. Hence, there is reduction in the 
solid migration from the wall to the core (i.e. low radial gas drag force) or solid carry over by the reversing gas (i.e. low upward gas drag force)

According to the above analysis, it is concluded that the gas velocity has little effect on the separator efficiency, at least within the operating conditions considered here. In biomass pyrolysis, however, the gas velocity has a critical effect on the product quality due to its effect on the gas and solid residence time. The residence time can be quantified through the average gas velocity, particularly within the GDH region, as discussed in Section 4.2. The interrelation between the gas velocity, gas/solid residence times and the GDH in a downer pyrolysis reactor is a complex one and requires careful optimization in order to achieve the best product quality.

\subsubsection{Effect of solid mass flow rate}

The effect of solid mass flow rate on the separator efficiency was investigated using four different flow rates of $m_{s}=0.004 \mathrm{~kg} / \mathrm{s}, 0.02 \mathrm{~kg} / \mathrm{s}, 0.04 \mathrm{~kg} / \mathrm{s}$ and $0.08 \mathrm{~kg} / \mathrm{s}$ at a fixed gas mass flow rate of $m_{g}=0.0039 \mathrm{~kg} / \mathrm{s}$. This corresponded to solid to gas flow ratios (solid loading) of $m_{s} / m_{g}=1,5,10$ and 20 respectively. All the other operating conditions were set to the default values. The experimental and predicted results, shown in Fig. 22, suggest that the separator efficiency improves as the solid loading increases within the range of $\dot{m}_{s} / \dot{m}_{g}<10$, beyond which the efficiency appears to be independent of solid loading. This trend is less pronounced in the predicted data, which show very limited changes. Quantitatively, there is an over-prediction of separator efficiency when compared with the experiment data; particularly at low solid loading.

Fig. 23 shows the velocity vectors as a function of the solid loading. In the wall region below the cone deflector, there is a clear change in the magnitude and direction of the gas velocity vector, particularly in the right hand side below the gas 
exit pipe. This implies an increased downward gas drag force, which positively adds to the solid gravity force. It is therefore concluded that as the solid loading increases the amount of solid entrained by the reversing gas at the central region below the cone deflector reduces. The solid concentration in the wall region massively increases while the core region remains relatively constant which can be seen in Fig. 24. The solid concentration and velocity profiles at the sample level, shown in Fig. 25 , indicate considerable hydrodynamic changes below the cone deflector as the solid loading increased. The gas velocity, however, shows exactly the opposite behavior with the axial velocity in the core region more than doubled when increasing the solid flow rare from 0.004 to $0.08 \mathrm{~kg} / \mathrm{s}$, while the velocity near the walls is slightly increased. Because the increase in the axial upward gas velocity in the centre takes place in a region that is at extremely low in solid concentration, the separator efficiency remains almost independent of the increase in solid loading. Accordingly, it is recommended to operate this downer reactor at a high solid flow rate for the following three main advantages:

i. Increased upward gas velocity towards the gas outlet pipe within the GDH region, therefore reducing the gas residence time in the reactor.

ii. Improved separator efficiency, as evident from the experimental and predicted results.

iii. Increasing the reactor processing capacity for biomass pyrolysis.

\subsubsection{Effect of the particle restitution and specularity coefficients}

It is understood that the particle size plays a major role on the separator efficiency such that the larger the particles size the higher the separator efficiency. Another important parameter of interest here is the degree of particle momentum loss or rebound upon hitting the solid surfaces, which is defined in the model through the restitution coefficient and specularity coefficient. The effective particle-wall restitution coefficient $\left(e_{s, w}\right)$ was determined experimentally by the co-authors from the ICFAR by measuring the rebound velocities of silica sand when hitting various types of solid 
surfaces; giving restitution coefficients ranging from 0.73 (Plexiglas surface) to 0.48 (paper surface). In this study, the same range of particle restitution coefficient was implemented in the model to investigate the effect of this parameter on the separator efficiency. The effect of the specularity coefficient $(\varphi)$ was investigated by using values of $\varphi=0,0.1$ and 1.0 , thus covering the two extreme ends of particle-wall interaction; free slip condition at $\varphi=0$ and no slip condition at $\varphi=1$. Reported studies (e.g. [25] ) have shown that the restitution coefficients have an effect on the solid velocity, gas velocity and solid concentration. It is also understood that as the restitution coefficient increases there is a corresponding increase in the wall shear stress. The specularity coefficient, on the other hand, has been reported to have a pronounced effect on the solid concentration, as increasing this parameter results in reducing the solid concentration at the wall.

Fig. 26 shows a comparison between the predicted and measured separator efficiency as a function of the particle restitution coefficient. It is clear that the predicted separator efficiency is a very weak function of this parameter. This is in good agreement with some of the reported literature (e.g. [25]) which suggest that the particle restitution coefficient (in the range 0.6 0.99) has limited effects on the solid velocity, gas velocity and the solid concentration in circulating fluidized bed reactors. The experimental data shows a slight decrease in the efficiency as the restitution coefficient decreases; however, this is still within a very limited range.

Fig. 27 shows the changes in the predicted separator efficiency with changing the value of the secularity coefficient. While there is clear drop in the efficiency as the specularity coefficient increases, this is still within a very limited range. This change is believed to be a result of the increase in the wall shear stress (no-slip condition), which in turn results in hindering the downward flow of the dense wall layer and hence giving rise to particle migration from the wall to the core followed by 
entrainment by the reversing gas towards the exit pipe. This phenomenon is demonstrated by the changes in the solid velocity and concentration profiles shown in Fig. 28. The specularity coefficient appears to have a significant effect on the gas velocity and solid concentration at the wall regions, which is in good agreement with the observation reported by Jin et al [25], and in spite of this there is a negligible effect on the separator efficiency.

\section{Conclusions}

The hydrodynamics in a downer pyrolysis reactor equipped with a novel gas-solid separator have been investigated theoretically using an Eulerian-Eulerian (two-fluid) CFD model. The novel separator, which consists of a cone deflector and a gas outlet pipe, was designed by the co-authors from the ICFAR (Canada). The model predictions were compared with experimental measurements of separator efficiency. This study revealed interesting hydrodynamic features around the cone deflector, where due to the restriction of the flow passage and solid deflection towards the walls, the region below the deflector in the downer reactor was completely transformed to behave like a riser, characterized by distinct upward gas flow at the core and dense falling solid layer at the walls. These distinct hydrodynamic features allowed for high efficiency of gas-solid separation up to $100 \%$. A new method for estimating the gas disengagement height $(\mathrm{GDH})$ was developed to help in estimating the gas residence time in this novel reactor. This study also included detailed sensitivity analysis of the separator efficiency towards the various operating conditions, including the effect of particle restitution and secularity coefficients. In the second part of this study, the present hydrodynamic model will be extended to include reaction kinetics and heat transfer to simulate the reactor thermochemical performance during the pyrolysis of biomass. 


\section{Notation}

$a$

A

B

C

$C_{D}$

$C_{\mu}, C_{1 \varepsilon}, C_{2 \varepsilon}$

$C_{f r, s_{i} s_{j}}$

$d_{S_{i}}$

$D_{C}$

$D_{g o}$

$e_{S_{i} s_{j}}$

$e_{s_{i}, w}$

$g$

$g_{0}$

$G_{k, g}$

$\overline{\bar{I}}$

$I_{2 D}$

$L_{1}, L_{2}$

$L_{S}$

$k_{g}$

$m_{\text {collected }}, m_{\text {in }}$

$\dot{m}_{g}, \dot{m}_{s}$

P
Gap between conical deflector and reactor wall (m)

Model parameter (-)

Model parameter (-)

Courant number (-)

Drag coefficient (-)

Constants (-)

Friction coefficient between solid phase $i$ and phase $j(-)$

Particle diameter of solid phase $i(\mathrm{~m})$

Reactor diameter $(\mathrm{m})$

Diameter of gas outlet pipe (m)

Particle-particle restitution coefficient (-)

Particle-wall restitution coefficient (-)

Gravity $\left(\mathrm{m} \mathrm{s}^{-2}\right)$

Radial distribution function (-)

Production of turbulent kinetic energy $\left(\mathrm{kg} \mathrm{m}^{-1} \mathrm{~s}^{-2}\right)$

Unit vector (-)

Second invariant of the deviatoric stress tensor $\left(\mathrm{s}^{-2}\right)$

Reactor dimension (m)

Separation length $(\mathrm{m})$

Turbulence kinetic energy $\left(\mathrm{m}^{2} \mathrm{~s}^{-2}\right)$

Mass of collected and fed solid particles respectively $(\mathrm{kg})$

Mass flow rate of gas and solid respectively $\left(\mathrm{kg} \mathrm{s}^{-1}\right)$

Pressure (pa) 


$\begin{array}{ll}\overline{\bar{S}} & \text { Strain rate }\left(\mathrm{s}^{-1}\right) \\ R e_{s_{i}} & \text { Reynolds number of solid phase } i(-) \\ t & \text { Time (s) } \\ \vec{u}_{g}, \vec{u}_{s_{i}} & \text { Gas and solid velocity vector }\left(\mathrm{m} \mathrm{s}^{-1}\right) \\ u_{s_{i}, w} & \text { Particle velocity at wall }\left(\mathrm{m} \mathrm{s}^{-1}\right) \\ v_{r, s_{i}} & \text { Terminal velocity correlation (-) } \\ X_{10}, X_{50}, X_{90} & \text { Particle size at accumulative volume fraction at } 10 \%, 50 \%, 90 \%\end{array}$

\section{Greek symbols}

$\alpha$

$\alpha_{g}, \alpha_{s_{i}}$

$\beta$

$\gamma_{\Theta_{s_{i}}}$

$\varepsilon_{g}$

$\eta$

$\Theta_{S_{i}}$

$\kappa_{\Theta_{s_{i}}}$

$\lambda_{s_{i}}$

$\mu_{l, g}, \mu_{t, g}$

$\mu_{s_{i}, \mathrm{col}}$

$\mu_{s_{i}, k i n}$

$\mu_{s_{i}, f r}$

$\Pi_{k, g}$

$\prod_{\varepsilon, g}$

$\rho_{s}, \rho_{g}$

$\overline{\bar{\tau}}$

$\sigma_{k}, \sigma_{\varepsilon}$

$\phi$

Angle of conical deflector (Degree)

Volume fraction of gas and solid phase $i$ respectively (-)

Momentum exchange coefficient $\left(\mathrm{kg} \mathrm{m}^{-3} \mathrm{~s}^{-1}\right)$

Collisional energy dissipation $\left(\mathrm{kg} \mathrm{m}^{-1} \mathrm{~s}^{-3}\right)$

Turbulent dissipation rate $\left(\mathrm{m}^{2} \mathrm{~s}^{-3}\right)$

Separation efficiency (-)

Granular temperature of solid phase $i\left(\mathrm{~m}^{2} \mathrm{~s}^{-2}\right)$

Diffusion coefficient of granular energy $\left(\mathrm{kg} \mathrm{m}^{-1} \mathrm{~s}^{-1}\right)$

Particle bulk viscosity $\left(\mathrm{kg} \mathrm{m}^{-1} \mathrm{~s}^{-1}\right)$

Viscosity of gas phase due to laminar, turbulent flow $\left(\mathrm{kg} \mathrm{m}^{-1} \mathrm{~s}^{-2}\right)$

Viscosity of solid phase $i$ due to collision $\left(\mathrm{kg} \mathrm{m}^{-1} \mathrm{~s}^{-1}\right)$

Viscosity of solid phase $i$ due to kinetics $\left(\mathrm{kg} \mathrm{m}^{-1} \mathrm{~s}^{-1}\right)$

Viscosity of solid phase $i$ due to friction $\left(\mathrm{kg} \mathrm{m}^{-1} \mathrm{~s}^{-1}\right)$

Influence of solid phases on gas phase $\left(\mathrm{m}^{2} \mathrm{~s}^{-3}\right)$

Influence of solid phases on gas phase $\left(\mathrm{m}^{2} \mathrm{~s}^{-4}\right)$

Solid and gas densities respectively $\left(\mathrm{kg} \mathrm{m}^{-3}\right)$

Shear stress tensor $\left(\mathrm{kg} \mathrm{m}^{-1} \mathrm{~s}^{-2}\right)$

Constants (-)

Angle of Internal friction (Degree) 
$\phi_{k s_{i}}$

$\varphi$

Energy exchange between phase $\mathrm{k}$ and solid phase $i\left(\mathrm{~kg} \mathrm{~m}^{-1} \mathrm{~s}^{-1}\right)$ Specularity coefficient (-)

Acknowledgement: The authors from the UK thank The Leverhulme Trust for a research grant (RPG-410).

\section{References}

[1] C. Mirgain, C. Briens, M. Del Pozo, R. Loutaty and M. Bergougnou, Modeling of feed vaporization in fluid catalytic cracking, Industrial \& Engineering Chemistry Research, 39 (2000) 4392-4399

[2] Y. Zhang, X. Wang, G. L. Riskowski, L. L. Christianson and S. E. Ford, Particle separation efficiency of a uniflow Deduster with different types of dusts, ASHRAE Transactions, 107 (2001) 93-98

[3] C. Huang, Z. Qian, M. H. Zhang and F. Wei, Solids mixing in a down-flow circulating fluidized bed of 0.418-m in diameter, Powder Technology, 161 (2006) 4852

[4] A. V. Bridgwater and G. V. C. Peacocke, Fast pyrolysis processes for biomass, Renewable \& Sustainable Energy Reviews, 4 (2000) 1-73

[5] E. Hoekstra, W. P. M. van Swaaij, S. R. A. Kersten and K. J. A. Hogendoorn, Fast pyrolysis in a novel wire-mesh reactor: Design and initial results, Chemical Engineering Journal, 191 (2012) 45-58

[6] M. I. Jahirul, M. G. Rasul, A. A. Chowdhury and N. Ashwath, Biofuels Production through Biomass Pyrolysis-A Technological Review, Energies, 5 (2012) 4952-5001 [7] M. Huard, C. Briens, F. Berruti and T. A. Gauthier, A Review of Rapid Gas-Solid Separation Techniques, International Journal of Chemical Reactor Engineering, 8 (2010)

[8] Y. Cheng, C. N. Wu, J. X. Zhu, F. Wei and Y. Jin, Downer reactor: From fundamental study to industrial application, Powder Technology, 183 (2008) 364-384 
[9] M. Huard, An investigation of a novel gas-solid separator for downer reactors, M.E.Sc. Thesis, The University of Western Ontario, London,Canada, (2009)

[10] M. Huard, F. Berruti and C. Briens, Experimental Study of a Novel Fast GasSolid Separator for Pyrolysis Reactors, International Journal of Chemical Reactor Engineering, 8 (2010)

[11] K. Ropelato, H. F. Meier and M. A. Cremasco, CFD study of gas-solid behavior in downer reactors: An Eulerian-Eulerian approach, Powder Technology, 154 (2005) 179-184

[12] Y. N. Kim, C. N. Wu and Y. Cheng, CFD simulation of hydrodynamics of gassolid multiphase flow in downer reactors: revisited, Chemical Engineering Science, 66 (2011) 5357-5365

[13] T. Samruamphianskun, P. Piumsomboon and B. Chalermsinsuwan, Computation of system turbulences and dispersion coefficients in circulating fluidized bed downer using CFD simulation, Chemical Engineering Research \& Design, 90 (2012) 21642178

[14] D. Winfield, M. Cross, N. Croft, D. Paddison and I. Craig, Performance comparison of a single and triple tangential inlet gas separation cyclone: A CFD Study, Powder Technology, 235 (2013) 520-531

[15] A. Kepa, Division of outlet flow in a cyclone vortex finder-The CFD calculations, Separation and Purification Technology, 75 (2010) 127-131

[16] K. W. Chu, B. Wang, D. L. Xu, Y. X. Chen and A. B. Yu, CFD-DEM simulation of the gas-solid flow in a cyclone separator, Chemical Engineering Science, 66 (2011) 834-847

[17] J. T. Jenkins and S. B. Savage, A Theory for the Rapid Flow of Identical, Smooth, Nearly Elastic, Spherical-Particles, Journal of Fluid Mechanics, 130 (1983) 187-202

[18] J. Ding and D. Gidaspow, A Bubbling Fluidization Model Using Kinetic-Theory of Granular Flow, Aiche Journal, 36 (1990) 523-538 
[19] S. M., The Particle-Particle Drag Term in a Multiparticle Model of Fluidization, National Technical Information Service, Springfield, VA. DOE/MC/21353-2373, NTIS/DE87006500, (1987)

[20] B. E. Launder and D. B. Spalding, Lectures in Mathematical Models of Turbulence, Academic press, London, (1972)

[21] S. A. VASQUEZ and V. A. IVANOV, A Phase Coupled Method for Solving Multiphase Problems on Unstructured Meshes, ASME 2000 Fluids Engineering Division Summer Meeting, (2000)

[22] R. Courant, K. Friedrichs and H. Lewy, On the partial difference equations of mathematical physics,, IBM Journal of Research and Development, 11 (1928) 215234

[23] P. C. Johnson and R. Jackson, Frictional-Collisional Constitutive Relations for Granular Materials, with Application to Plane Shearing, Journal of Fluid Mechanics, 176 (1987) 67-93

[24] D. Geldart, Y. Xue and H. Y. Xie, Measurement of bottom bed and transport disengaging heights in beds of fresh FCC catalyst, AIChE symposium series, 91 (1995) 93-101

[25] B. S. Jin, X. F. Wang, W. Q. Zhong, H. Tao, B. Ren and R. Xiao, Modeling on High-Flux Circulating Fluidized Bed with Geldart Group B Particles by Kinetic Theory of Granular Flow, Energy \& Fuels, 24 (2010) 3159-3172 


\title{
A CFD study of biomass pyrolysis in a downer reactor equipped with a novel gas-solid separator - I: hydrodynamic performance
}

\author{
Xi Yu ${ }^{1}$, Yassir Makkawi ${ }^{1 *}$, Raffaella Ocone ${ }^{2}$, Martin Huard ${ }^{3}$, Cedric Briens ${ }^{3}$ and \\ Franco Berruti ${ }^{3}$
}

1 European Bioenergy Research Institute (EBRI), School of Engineering and Applied Science, Aston University, Birmingham B4 7ET, United Kingdom

2 Chemical Engineering, Heriot-Watt University, Edinburgh EH14 4AS, UK

3 The Institute for Chemicals and Fuels from Alternative Resources (ICFAR), Western University, London, Canada

\section{Abstract:}

This study presents the first part of a CFD study on the performance of a downer reactor for biomass pyrolysis. The reactor was equipped with a novel gas-solid separation method, developed by the co-authors from the ICFAR (Canada). The separator, which was designed to allow for fast separation of clean pyrolysis gas, consisted of a cone deflector and a gas exit pipe installed inside the downer reactor. A multi-fluid model (Eulerian-Eulerian) with constitutive relations adopted from the kinetic theory of granular flow was used to simulate the multiphase flow. The effects of the various parameters including operation conditions, separator geometry and particle properties on the overall hydrodynamics and separation efficiency were investigated. The model prediction of the separator efficiency was compared with experimental measurements. The results revealed distinct hydrodynamic features around the cone separator, allowing for up to $100 \%$ separation efficiency. The developed model provided a platform for the second part of the study, where the biomass pyrolysis is simulated and the product quality as a function of operating

\footnotetext{
* Corresponding author. +44 (0)121 204 3398; email: y.makkawi@aston.ac.uk
} 
conditions is analysed.

Key words: Biomass pyrolysis, downer reactor, gas-solid separation, CFD modeling, hydrodynamics

\section{Introduction}

The escalating global concern over the exhaustion of non-renewable energy sources lead to the recent development of a range of novel technologies for the use of renewable energy resources, such as biomass, solar and wind. Among these resources and technologies, biomass pyrolysis has emerged as a very promising renewable alternative for bio-oil production. In a large commercial scale, this could be carried out in a dual fluidized bed (DFB) system with various optional arrangements. The schematics in Fig. 1 demonstrate examples of these arrangements. In this study, we are interested in the downer-riser type of a dual fluidized bed, shown in Fig. 1-b, where the biomass pyrolysis takes place in the downer side of the reactor, while the riser side is used for combustion, thus providing the heat required for the pyrolysis through the circulating inert heat carrier solid (such as sand). This arrangement has the following specific advantages for bio-oil production through fast pyrolysis:

i. The downer pyrolysis reactor can be operated with very low carrier gas (e.g. nitrogen) flow rates, which is desirable in some cases to reduce up-stream pre-heating and downstream processing.

ii. Reducing the gas and solid back-mixing [1, 2, 3] thus, limiting the spread of the gas/solid residence time distribution, i.e. near to plug flow.

iii. Relatively low cost, simple operation/control and high energy efficiency.

iv. The char combustion in the second reactor will guarantee sustainable operation and better control of the pyrolysis temperature in the first reactor.

However, in order to achieve high conversion efficiency (more than $70 \%$ bio-oil yield) in a downer reactor there remains two main technical challenges: 
i. Control of the pyrolysis gas residence time within the hot zone of the reactor (ideally 1-2 seconds). Longer residence time of the pyrolysis gas at high temperature initiates a range of undesirable side reactions, which could adversely affect the quality of the product bio-oil $[4,5]$

ii. Control of the downstream contact between the pyrolysis gas and bio-char. The bio-char, formed during pyrolysis, acts as a vapor cracking catalyst, therefore should be separated as soon as the pyrolysis vapor is released [6]

Char, as well as other entrained fine particles, can primarily be separated from the pyrolysis gas by using conventional cyclones (reverse and co-current flow types). However, this carries the risk of increasing the contact time between the gas and char inside the cyclone. In addition, the cyclone inlet is commonly placed external to the reactor or away from the pyrolysis zone, thus, causing extra contact time between the solid and gas. The extensive review conducted by Huard et al. [7] and Cheng et al. [8] on downer reactors and rapid gas-solid separation techniques revealed that there are limited attempts on implementing new design methods for rapid gas-solid separation in these reactors.

Recent research at the Institute for Chemicals and Fuels from Alternative Resources (ICFAR) has led to the development of a novel gas-solid separation device for a downer pyrolysis reactor. The device features a cone-shaped solid deflector positioned above a gas outlet pipe, both positioned concentrically in the downer pipe (see Fig. 2). This was designed to achieve primary solid-gas separation and gas removal within the same device [9] The separator allows for better control of the pyrolysis vapor residence time, therefore, reducing the severity of vapor overcracking compared to other fast separation methods. Experimental work by Huard et al. [10] has shown that this separator can achieve very high solid-gas separator efficiency above $99.99 \%$ when using spherical silica sand particles of $200 \mu \mathrm{m}$ 
diameter.

Computational Fluid Dynamics (CFD) modeling is one of the powerful tools to analyse gas-solid flow behavior, including that involves intense heat transfer and chemical reactions. The co-authors from the ICFAR have previously used an Eulerian-Lagrangian modeling approach to investigate the effect of the particle elasticity on the separator efficiency in the same novel separator investigated in this study [9]. While this approach revealed important details of the particle-wall collision and its effects on the separator efficiency and mechanism, the simulation domain was limited to the separator zone only and the total solid volume fraction was limited to a maximum of $4 \times 10^{-5}$. The Eulerian-Eulerian (also referred to as two-fluid) is another modeling approach that has the advantage of being robust and realistic in computational time, especially when considering a large number of particles or large simulation domain. Unlike the Eulerian-Lagrangian approach, which treats each single particle as a dispersed phase in the continuum fluid flow, the Eulerian-Eulerian approach treats both of the fluid and solid phases as an interpenetrating continuum. Studies on the Eulerian-Eulerian simulation of solid-gas hydrodynamics in a downer reactor have been previously reported by Ropelato et al. [11], Kim et al [12] and Samruamphianskun [13] This modeling approach was found to be especially useful in predicting the effects of inlet design and flow conditions on the solid distribution and dispersion behavior. This CFD modeling approach has also been used by different researchers to study the phenomena of solid-gas separation in cyclones $[14,15,16]$

In this study, the main objectives are:

i. to develop a valid Eulerian-Eulerian (multi-fluid) CFD model capably of predicting the detailed hydrodynamic behaviour in a downer reactor equipped with a novel gas-solid separation device; 
ii. to use the developed model in investigating the effect of the operating conditions and various separator design parameters on the overall hydrodynamics, with particular focus on the separator efficiency;

iii. to provide a platform for the development of a predictive model of the pyrolysis reactions and yield in the downer reactor equipped with the novel gas-solid separator.

The investigation was carried out theoretically and experimentally in a cold flow reactor model equipped with the ICFAR novel gas-solid separator and gas removal mechanism, as described in details in the experimental section. The theoretical transient model was solved in three-dimensional coordinates using the EulerianEulerian (two-fluid) approach, employing constitutive relations from the kinetic theory of granular flow (KTGF) [17]. In the second part of this study, the developed hydrodynamic model will be extended to include heat transfer and reaction kinetics to demonstrate the advantages of the ICFAR separator in improving the performance and product quality in a biomass downer pyrolysis reactor.

\section{Experiments and procedure}

The experimental work described here was carried out by the co-investigators at the ICFAR in Canada. The equipment consisted of a cold flow gas-solid flow downer of $133.5 \mathrm{~cm}$ height and $7.0 \mathrm{~cm}$ diameter, equipped with the ICFAR novel gas-solid separator as shown in Fig. 2 and 3. This separator included a gas removal pipe and a cone deflector, where the bottom of the deflector and tip of the pipe were located $98.6 \mathrm{~cm}$ below the downer inlet. A solid collection tank of $20.4 \mathrm{~cm}$ diameter and 21.8 $\mathrm{cm}$ height was placed at the bottom of the downer column around $34.9 \mathrm{~cm}$ below the cone deflector. Compressed air at room temperature was supplied to the downer from a bank of calibrated sonic orifice nozzles. The Sauter mean diameter of the particle mixture used was $188 \mu \mathrm{m}$ and its skeletal density was $2650 \mathrm{~kg} / \mathrm{m}^{3}$. The 
particle size distribution of the mixture is shown in Fig. 4.

The solid particles were delivered to the downer column from an air pressurized tank mounted above the downer main air inlet. The total mass flowrate of air in the downer was $\dot{m}_{g}=0.0039 \mathrm{~kg} / \mathrm{s}$, which corresponded to a superficial gas velocity of $U_{g}$ $=0.73 \mathrm{~m} / \mathrm{s}$. The solids mass flowrate was adjusted by changing the feed tank air pressure and this was varied between $\dot{m}_{s}=0.017 \mathrm{~kg} / \mathrm{s}$ and $0.083 \mathrm{~kg} / \mathrm{s}$, which corresponded to solids-to-gas loading ratios of $\dot{m}_{s} / \dot{m}_{g}=4.3$ to 21 . The gas-solids mixture flowed co-currently in the downer before entering the gas-solids separation zone. Three different cone deflectors with various internal angles of $60^{\circ}, 90^{\circ}$ and $120^{\circ}$ were used. The downward falling particles were collected in the tank at the bottom of the unit, while the gas stream, along with any entrained particles, exited the system through the gas outlet pipe mounted in the centre of the downer cross section and below the cone deflector. A bag filter connected at the end of the gas exhaust line was used to collect the particles entrained in the exiting gas stream.

At the start of each experiment, the total mass of solids fed into the system, $m_{i n}$, was measured. The mass of the entrained solids collected in the bag filter, $m_{\text {collected, }}$ was then measured at the end of each experiment. Thus, the experimental percentage total solids separation efficiency, $\eta$, was calculated from the following expression:

$\eta=\left(1-\frac{m_{\text {collected }}}{m_{\text {in }}}\right) \times 100 \%$

The mass flowrate of solids was determined by measuring the total mass of solid collected in the filter bag and tank against the recorded time. 


\section{Hydrodynamic model}

The overall reactor hydrodynamics and gas-solid separation was investigated using the Eulerian-Eulerian (multi-fluid) model approach based on the Kinetic Theory of Granular Flow (KTGF). The developed model was solved using the CFD software ANSYS FLUENT (Ver. 14). In order to mimic the wide size distribution of the solid mixture used in the experiment, the simulation was carried out using a solid mixture of three different particle sizes, as detailed in section 3.3. The main model equations for non-reacting isothermal gas-solid flow are given by:

Continuity equations:

$\frac{\partial\left(\alpha_{g} \rho_{g}\right)}{\partial t}+\nabla\left(\alpha_{g} \rho_{g} \vec{u}_{g}\right)=0$

$\frac{\partial\left(\alpha_{s_{i}} \rho_{s}\right)}{\partial t}+\nabla\left(\alpha_{s_{i}} \rho_{s} \vec{u}_{s_{i}}\right)=0$

$\sum_{i=1}^{3} \alpha_{s_{i}}+\alpha_{g}=1$

Momentum equations:

$$
\begin{aligned}
& \frac{\partial\left(\alpha_{g} \rho_{g} \vec{u}_{g}\right)}{\partial t}+\nabla\left(\alpha_{g} \rho_{g} \vec{u}_{g} \vec{u}_{g}\right)=-\alpha_{g} \nabla P+\nabla \overline{\bar{\tau}}_{g}-\sum_{i=1}^{3} \beta_{g s_{i}}\left(\vec{u}_{g}-\vec{u}_{s_{i}}\right)+\alpha_{g} \rho_{g} g \\
& \frac{\partial\left(\alpha_{s_{i}} \rho_{s} \vec{u}_{s_{i}}\right)}{\partial t}+\nabla\left(\alpha_{s_{i}} \rho_{s} \vec{u}_{s_{i}} \vec{u}_{s_{i}}\right)=-\alpha_{s_{i}} \nabla P-\nabla P_{s_{i}}+\nabla \overline{\bar{\tau}}_{s_{i}}+\beta_{g s_{i}}\left(\vec{u}_{g}-\vec{u}_{s_{i}}\right) \\
& +\sum_{j=1, j \neq i}^{3} \beta_{s_{i} s_{j}}\left(\vec{u}_{s_{j}}-\vec{u}_{s_{i}}\right)+\alpha_{s_{i}} \rho_{s} g
\end{aligned}
$$

where:

$$
\begin{aligned}
& \overline{\bar{\tau}}_{k}=\left(\lambda_{k}-\frac{2}{3} \mu_{k}\right)\left(\nabla \cdot \vec{u}_{k}\right) \overline{\bar{I}}+2 \mu_{k} \overline{\bar{S}}_{k} \\
& \overline{\bar{S}}_{k}=\frac{1}{2}\left(\nabla \vec{u}_{k}+\left(\nabla \vec{u}_{k}\right)^{T}\right)
\end{aligned}
$$

$k$ represents solid or gas phase.

To obtain the granular temperature, the FLUENT code was optionally set to use a partial differential equation (Pseudo Energy Equation) as follows [18]: 


$$
\begin{aligned}
\frac{3}{2}\left[\frac{\partial\left(\alpha_{s_{i}} \rho_{s} \Theta_{s_{i}}\right)}{\partial t}\right. & \left.+\nabla\left(\alpha_{s_{i}} \rho_{s} \Theta_{s_{i}}\right) \vec{u}_{s_{i}}\right] \\
& =\left(-P_{s_{i}} \overline{\bar{I}}+\overline{\bar{\tau}}_{s_{i}}\right): \nabla \vec{u}_{s_{i}}+\nabla\left(\kappa_{T} \nabla \Theta_{s_{i}}\right)-\gamma_{T}+\sum_{k=1}^{3} \phi_{k s_{i}}
\end{aligned}
$$

The various closure and constitutive relations used in the model are given in Table 2. In order to take into consideration the solid-solid frictional stresses at the dense regions of the reactor, the friction equation proposed by Schaeffer [19], as given in Equation T1-5, was used. Due to the highly turbulence of the flow near the deflector zone the standard K-epsilon turbulence and energy dissipation equations proposed by Launder and Spalding [20] were also incorporated in the model and these are given as follows:

Turbulence momentum equations:

$\frac{\partial\left(\alpha_{g} \rho_{g} k_{g}\right)}{\partial t}+\nabla\left(\alpha_{g} \rho_{g} \vec{u}_{g} k_{g}\right)=\alpha_{g} G_{k, g}+\nabla\left(\alpha_{g} \frac{\mu_{t, g}}{\sigma_{k}} k_{g}\right)-\alpha_{g} \rho_{g} \varepsilon_{g}+\alpha_{g} \rho_{g} \Pi_{k, g}$

Turbulent kinetic energy dissipation equation:

$\frac{\partial\left(\alpha_{g} \rho_{g} \varepsilon_{g}\right)}{\partial t}+\nabla\left(\alpha_{g} \rho_{g} \vec{u}_{g} \varepsilon_{g}\right)=\nabla\left(\alpha_{g} \frac{\mu_{t, g}}{\sigma_{\varepsilon}} \varepsilon_{g}\right)+\alpha_{g} \frac{\varepsilon_{g}}{k_{g}}\left(C_{1 \varepsilon} G_{k, g}-C_{2 \varepsilon} \rho_{g} \varepsilon_{g}\right)+\alpha_{g} \rho_{g} \Pi_{\varepsilon, g}$

where:

$$
\begin{aligned}
& G_{k, g}=\mu_{t, g}\left(\nabla \vec{u}_{g}+\left(\nabla \vec{u}_{g}\right)^{T}\right): \nabla \vec{u}_{g} \\
& C_{\mu}=0.09, C_{1 \varepsilon}=1.44, C_{2 \varepsilon}=1.92, \sigma_{k}=1, \sigma_{\varepsilon}=1.3
\end{aligned}
$$

\subsection{Computational domain and meshing}

Fig. 5 shows the computational domain and the meshing used in solving the model. This was generated using a finite volume method with hybrid cells of structured and unstructured grids, giving a total of 30,785 cells. In order to capture the steep hydrodynamic variations around the walls of the separation device (the conical deflector and the gas exit pipe), the grid size was refined by setting the minimum and 
maximum grid size at 0.3 and $1.0 \mathrm{~cm}$ respectively. In the rest of the simulation domain the minimum and maximum grid size was set at 1.0 and $5.0 \mathrm{~cm}$ respectively. The impact of the grid size on the solution accuracy was initially tested by setting three different meshing schemes and the grid size used in this study was found to give acceptable grid independent solution.

\subsection{Computation procedure}

The model equations were solved using the finite volume approach. First-order discretization schemes were used for the solution of the convection terms in all governing equations. The relative error between any two successive iterations was specified by using a convergence criterion of $10^{-3}$ for each scaled residual component. The phase-coupled SIMPLE (PC-SIMPLE) algorithm [21], which is an extension of the SIMPLE algorithm to multiphase flows, was applied for the pressure-velocity coupling. The linearized equations for governing equations were solved using a block algebraic multigrid method. In order to ensure easy convergence of the various partial differential equations (PDE) in the model, the Courant-Friedrichs-Lewy (CFL) condition for three-dimensional PDE is followed:

$$
\mathrm{C}=\frac{\mathrm{u}_{\mathrm{x}} \Delta \mathrm{t}}{\Delta \mathrm{x}}+\frac{\mathrm{u}_{\mathrm{y}} \Delta \mathrm{t}}{\Delta \mathrm{y}}+\frac{\mathrm{u}_{\mathrm{z}} \Delta \mathrm{t}}{\Delta \mathrm{z}} \leq \mathrm{C}_{\max }
$$

where $C_{\max }$ is specified by the CFL condition to fall within the range of $\sim 1-5$ [22]. In this study, a time step of 0.005 seconds was found to satisfy this condition.

\subsection{Boundary and simulation conditions}

The particle-wall restitution coefficient and the specularity coefficient are two important parameters in determining the dynamics of particles at the wall region. The following wall boundary conditions were employed in the model [23]:

$$
u_{s_{i}, w}=-\frac{6 \mu_{s_{i}} \alpha_{s, \max }}{\sqrt{3 \Theta_{s_{i}}} \pi \varphi \rho_{s} \alpha_{s_{i}} g_{0, s_{i} s_{i}}} \frac{\delta u_{s_{i}, w}}{\delta n}
$$


$\Theta_{s_{i}}=-\frac{k_{s_{i}} \Theta_{s_{i}}}{\gamma_{w}} \frac{\delta \Theta_{s_{i}, w}}{\delta n}+\frac{\sqrt{3} \pi \varphi \rho_{s} \alpha_{s_{i}} u_{s_{i}, s l i p}^{2} g_{0, s_{i} s_{i}} \Theta_{s_{i}}{ }^{\frac{3}{2}}}{6 \alpha_{s, \max } \gamma_{w}}$

$\gamma_{w}=\frac{\sqrt{3} \pi\left(1-e_{s_{i}, w}^{2}\right) \rho_{s} \alpha_{s_{i}} g_{0, s_{i} s_{i}} \Theta_{S_{i}}{ }^{\frac{3}{2}}}{4 \alpha_{s, \max }}$

where $\varphi$ is the specularity coefficient and $e_{s_{i}, w}$ is the particle-wall restitution coefficient. In order to reasonably match the particle size distribution used in the experiments, the simulations were carried out assuming the solid mixture to consist of three different particle sizes. The fraction of each particle size group was estimated from the experimental size distribution given in Fig. 4. The simulation particle sizes and percentages are given in Table 3. For the gas phase, the velocity at the wall was assumed zero (no slip condition). Table 4 summarizes the various operating conditions considered in the simulations. Some of these conditions were carefully selected to allow for the comparison of the model predictions with the corresponding experimental data.

\section{Results and discussion}

\subsection{Mechanism of gas-solid separation}

It is postulated that the drag and gravity forces, the last two terms in the left hand side of Eq. 3b, are the main forces dominating the hydrodynamic behavior of the gas-solid phases within the separator zone. Fig. 6 gives an overall description of the flow structure with close zoom-in at the cone deflector region. The gas velocity in the gap between the deflector and the wall is very high due to the considerable pressure drop, similar to gas expansion through a throttling device. In the region under the cone and below the gas exit pipe there is an upward gas drag force due to the high reverse gas phase velocity. However, the extremely dilute solid concentration in this zone means very little solids are being entrained. It is therefore desirable to minimize the upward gas drag force in this region in order to achieve high separator efficiency. On top of the cone deflector, the solid phase is diverted radially towards the wall and 
then accelerates through the gap between the deflector and the downer wall, pushed by a strong gas drag force. The influence of the gravity force in this region is also significant due to the high solid concentration. Accordingly, it is believed that any particles entrained through the exit pipe are falling under the influence of two different drag mechanisms:

i. Reverse gas flow (upward) under the cone deflector due to the abrupt gas pressure drop at the tip of the gas exit pipe. This makes the tip of the exit pipe act as a vacuum to the surrounding solids.

ii. Radial gas flow from the walls towards the core in the region just below the cone deflector. This results in the solids being first dragged towards the core, and then further dragged/sucked by the gas leaving through the exit pipe.

This is to some extent similar to the solid-gas separation mechanism in a cyclone, where in both cases the reverse gas flow in the core is responsible of solid entrainment. However, in the cyclone, the particles move radially towards the walls under the influence of centrifugal forces, while in the cone deflector, the particles are deflected radially by the cone wall to fall under the strong downward gas drag force in the "throttling" gap, as described earlier. It is worth noting that the modeling results reported by the co-authors from the ICFAR suggested that the solid rebound upon hitting the walls, investigated through changing the wall-particle restitution coefficient, may have a dominant role in the mechanism of gas-solid separation in the cone deflector. This hypothesis will be discussed in some details in the following sections.

Fig. 7 shows the predicted solid concentration and the gas velocity profiles at the level of $3.9 \mathrm{~cm}$ below the tip of the gas exit pipe (see Fig. $6 \mathrm{~b}$ for the sampling line level). These profiles reveals a very interesting hydrodynamic behavior where the solid concentration profile is shown to take the shape of a dense-wall and dilute- 
core, while the gas velocity takes the shape of an upward parabolic flow profile at the core and a downward flow at the walls. It appears that, due to the existence of the cone deflector, the overall flow pattern below this device has been completely changed from the classic gas-solid down flow pattern, commonly observed in downer reactors, to a more complex flow similar to that existing in a turbulent solid-gas flow riser.

\subsection{Gas disengagement height (GDH)}

Fig. 8 shows that there are four distinct flow zones each with characteristic flow behavior. These are mainly arising from the changes induced by the cone deflector and these can be described as flows:

Zone I: This is where fully developed flow and uniform distribution of the solid and gas phases take place, typical to that observed in a conventional downer reactor.

Zone II: This the where both of the solid and gas phases are first hitting the inclined plane to create a dense moving solid layer at the cone walls before being pushing by a strong gas drag force through the gap between deflector and the downer walls. Under the cone, the lowest solid concentration in the whole system exists and the gas is removed through the exit pipe driven by the rapid pressure drop at the exit pipe tip.

Zone III: This is where the disengagement of gas from the gas-solid flow mixture takes place. The overall flow hydrodynamics in this region is very complex due to the effect of sharp changes in pressure, which consequently leads to reverse gas flow towards the top and radial solid movement from the dense walls towards the dilute core.

Zone IV: This is where the solid phase is mainly concentrated at the walls. The radial flow diminishes and the particles fall under the strong influence of the gravity force before entering the solid collection tank at the bottom of the downer system. 
As described earlier, the main objective of the cone separator is to allow for fast and efficient separation of the gas from the downward gas-solid flow stream. In a biomass pyrolysis downer reactor, this separation should ideally take place immediately at the level of the gas outlet pipe tip and with zero solid entrainment. The first reason is to prevent undesirable secondary gas reactions by removing the gas from the reactor hot zone, and second, to prevent catalytic char cracking by limiting the contacts between the solid and gas. However, in reality, the gas separation from the solid-gas stream takes place a little further down beyond the level of the tip of the gas removal pipe. It is therefore particularly interesting to quantify the height of Zone III, in which the gas separation takes place. This is defined here as the gas disengagement height (GDH), analogous to the definition of the transport disengaging height (TDH) in gas-solid fluidized beds. The method used in this study to estimate the GDH is demonstrated in Fig. 9. The GDH is defined at the intercept of the lines tangential to the low pressure gradient curve and the steep changing pressure gradient curve, or alternatively, the GDH can be estimated from plotting the axial gas velocity against height as shown in Fig. 9-b. The pressure gradient method is similar to the method used by Geldart et al. [24] in determining the transport disengaging height $(\mathrm{TDH})$.

Fig. 10 shows the result of a sensitivity analysis of the GDH to a range of operating conditions. Please note that the y-axis in Fig. 10 represents the summation of the the $\mathrm{GDH}$ and the separation distance $L_{s}$, where the separation distance is defined as the distance from the cone rim to the tip of the gas exit pipe. The GDH range under the various operation conditions considered in the simulation was found to fall between $2.5 \mathrm{~cm}$ and $6 \mathrm{~cm}$. It is clear that the $\mathrm{GDH}$ is most sensitive to the solid loading and the solid flow rate. The increase in the separation distance from $0 \mathrm{~cm}$ to $7 \mathrm{~cm}$ and the solid flow rate from $0.004 \mathrm{~kg} / \mathrm{s}$ to $0.08 \mathrm{~kg} / \mathrm{s}$ caused a corresponding increase in GDH of around $30 \%$ for both cases. Clearly, the cone angle and gas flow rate appear 
to cause negligible effect in this regard. It should be noted that, while it is desirable to decrease the GDH as discussed earlier, this does not necessarily mean improving the separation efficiency, as will be demonstrated in the next section.

\subsection{Separator efficiency}

The theoretical separator efficiency was obtained by dividing the predicted solid flow rate at the gas exit pipe (entrained solids) by the inlet solid mass flow rate, such that,

$\eta=\frac{\dot{m}_{s, \text { exit }}}{\dot{m}_{s, i n}} \times 100 \%$

The separator efficiency was analysed with respect to various operating conditions. This also included a sensitivity analysis of the separation efficiency towards varying the wall-particle interactions mechanism, through changing the particle-wall restitution coefficient and specularity coefficient. Both parameters appear in the solid boundary condition of Eq. 8-9. The first coefficient is a measure of the degree of energy loss when the particles hit the walls, hence determining the rebound velocity, and the second coefficient defines the angle of rebound. It is therefore possible to determine the effect of the wall surface material and particle properties on the separation efficiency through changing these two parameters in the model.

In this study, $100 \%$ separator efficiency was obtained when operating with: large particle size of $d_{p}=328 \mu \mathrm{m}$, separation length $L_{s}=0 \mathrm{~cm}$, cone angle $\theta=60^{\circ}$, gas mass flow rate $m_{g}=0.0039 \mathrm{~kg} / \mathrm{s}$ and high solid flow rate $m_{s}=0.08 \mathrm{~kg} / \mathrm{s}$. This was found to dramatically decrease when decreasing the particle size. This result is in good agreement with the experimental study by Huard et al [10] where it was shown that the separator efficiency, when using FCC catalyst of $d_{p}=43 \mu \mathrm{m}$ and glass beads of $d_{p}=63 \mu \mathrm{m}$, is much lower than that achieved with sand of $d_{p}=200 \mu \mathrm{m}$. Fig. 11 shows the values of the predicted separator efficiency obtained within the range of operating conditions considered in this study. The detailed results and 
discussion on the effect of each of these parameters on the overall hydrodynamics and separator efficiency are given in the next sections. According to this data, it is concluded that the sensitivity of the separator efficiency towards the operating conditions can be ranked in order of decreasing impact on the separation efficiency as follows; (1) separation length (2) cone angle (3) gas flow rate (4) solid flow rate (5) particle physical properties (expressed in terms of the restitution and specularity coefficients). Note that, the impact of the particle size on the separation efficiency comes on top of all the above parameters.

\subsubsection{Effect of the separation length $\left(L_{s}\right)$}

The effect of separation distance on the separator efficiency was studied using three different separation lengths of $0 \mathrm{~cm}, 3.5 \mathrm{~cm}$ and $7 \mathrm{~cm}$, which corresponds to the normalized separation length $\left(L_{s} / D\right)$ of $0,0.5$ and 1 respectively. All the other parameters were set at the default values.

Figure 12 shows the separator efficiency as a function of the normalized separation length. The overall trend indicates a negative impact on the separator efficiency. The maximum mean efficiency was $99.986 \%$ and this dropped to $99.633 \%$ at $L_{s} / D=1$. The greater separation efficiency achieved with the particle size of $206 \mu \mathrm{m}$ compared to the size group of $324 \mu \mathrm{m}$ can be explained by the fact that the concentration of this particle group (60 wt\%) was greater than the latter one (20 wt\%). Therefore, the more frequent particle-particle interaction within the same group can neutralized part of radial velocity which may cause entrainment of particle. The same phenomenon was observed in Fig 15. In terms of sensitivity, the effect of the separation length on the separation efficiency is the highest compared to the other parameters investigated, as shown earlier in Fig. 11. It was also demonstrated earlier that the separation distance has also a relatively high effect on the GDH. The velocity vectors shown in Fig. 13 indicate that the increase in $L_{s}$ resulted in the creation of two vortices in the space between the cone deflector and the tip of the gas exit pipe. This 
can be attributed to the strong radial gas flow in this region, resulting from the considerable pressure drop at the exit pipe.

Fig. 14 shows the changes in the solid concentration and velocity profiles with changing the separation length at the sample level of $3.9 \mathrm{~cm}$ below the bottom of the cone deflector. It is clear that the solid concentration increases with increasing $L_{s}$, while the vertical upward gas velocity at the core decreases. This suggests that the upward gas drag force may have limited influence on the separation efficiency. It is the increased radial gas velocity (radial drag), the subsequent formation of vortices and the increased solids concentration at the core that collectively play the dominant role in decreasing the separator efficiency as the separation length increases.

\subsubsection{Effect of the cone deflector angle $(\theta)$}

The effect of the cone deflector angle on the separator efficiency was studied using various angles $\theta=60^{\circ}, 90^{\circ}$ and $120^{\circ}$. All other operating conditions were set at the default values. Fig. 15 shows that the separator efficiency decreases with increasing the cone angle. The maximum mean separator efficiency (taking into account the three particle sizes) was $99.986 \%$, this dropped to $99.869 \%$ efficiency when the angle is increased to $120^{\circ}$. This trend is in satisfactory agreement with the experimental data of Huard et al. [10]; however, the experiments showed less pronounced changes compared to the predictions, and this may be attributed to the differences between the particle size distribution in the experiment and the assumed size mixture in the model.

In Fig. 16 the magnitude and direction of the gas velocity vectors suggest that as the cone angle increases there is a greater chance the particles rebound more in the reverse direction from the cone inner surface and normal to the gas exit. This would slow down the particles and make them easier to be entrained, thus having a negative impact on the separator efficiency. In Fig. 17 there is clear increase in the 
solid concentration on top of the cone's upper surface due to flattering of the cone external surface as shown in Fig. 17; however this is not expected to have contributed to the change in the separation mechanism or efficiency.

\subsubsection{Effect of the gas mass flow rate}

The effect of inlet gas mass flow rate on the gas separator efficiency was investigated at three different flow rates of $0.0039 \mathrm{~kg} / \mathrm{s}, 0.0239 \mathrm{~kg} / \mathrm{s}$ and $0.0439 \mathrm{~kg} / \mathrm{s}$ and a fixed solid flow rate of $0.004 \mathrm{~kg} / \mathrm{s}$. This corresponds to inlet gas velocities of $0.73 \mathrm{~m} / \mathrm{s}, 4.5 \mathrm{~m} / \mathrm{s}$ and $8.2 \mathrm{~m} / \mathrm{s}$, respectively. All other operating conditions were set to the default values. Fig. 18 shows that the effect of the gas mass flow rate on the separator efficiency is negligible. This is in good agreement with the experimental observation reported by Huard et al. [10].

To gain further understanding on the effect of gas flow rate on the overall hydrodynamics, Fig. 19 shows the gas velocity vectors as function of the gas mass flow rate. It is clear that there is a significant change in the magnitude of the gas velocity but little change in the flow pattern. There is also evidence of a significant change in the solid concentration around the cone deflector as shown in Fig. 20. Despite this, such a dramatic change caused no effect on the separator efficiency due to counterbalance of forces, which are described as follows:

i. At a high gas velocity, there is considerable increase in the pressure drop between the gas exit pipe and its surroundings, hence high upward gas velocity (drag force), as shown in Fig. 21a. However, this is counterbalanced by the considerable reduction in the solid concentration in the wall and the core region below the exit pipe, as shown in Fig. 21b.

ii. At a low gas velocity, there is high solid concentration at the wall (i.e. high gravity force), as shown in Fig. 21b. This is associated with low pressure drop between the wall and the tip of the exit pipe. Hence, there is reduction in the 
solid migration from the wall to the core (i.e. low radial gas drag force) or solid carry over by the reversing gas (i.e. low upward gas drag force)

According to the above analysis, it is concluded that the gas velocity has little effect on the separator efficiency, at least within the operating conditions considered here. In biomass pyrolysis, however, the gas velocity has a critical effect on the product quality due to its effect on the gas and solid residence time. The residence time can be quantified through the average gas velocity, particularly within the GDH region, as discussed in Section 4.2. The interrelation between the gas velocity, gas/solid residence times and the GDH in a downer pyrolysis reactor is a complex one and requires careful optimization in order to achieve the best product quality.

\subsubsection{Effect of solid mass flow rate}

The effect of solid mass flow rate on the separator efficiency was investigated using four different flow rates of $m_{s}=0.004 \mathrm{~kg} / \mathrm{s}, 0.02 \mathrm{~kg} / \mathrm{s}, 0.04 \mathrm{~kg} / \mathrm{s}$ and $0.08 \mathrm{~kg} / \mathrm{s}$ at a fixed gas mass flow rate of $m_{g}=0.0039 \mathrm{~kg} / \mathrm{s}$. This corresponded to solid to gas flow ratios (solid loading) of $m_{s} / m_{g}=1,5,10$ and 20 respectively. All the other operating conditions were set to the default values. The experimental and predicted results, shown in Fig. 22, suggest that the separator efficiency improves as the solid loading increases within the range of $\dot{m}_{s} / \dot{m}_{g}<10$, beyond which the efficiency appears to be independent of solid loading. This trend is less pronounced in the predicted data, which show very limited changes. Quantitatively, there is an over-prediction of separator efficiency when compared with the experiment data; particularly at low solid loading.

Fig. 23 shows the velocity vectors as a function of the solid loading. In the wall region below the cone deflector, there is a clear change in the magnitude and direction of the gas velocity vector, particularly in the right hand side below the gas 
exit pipe. This implies an increased downward gas drag force, which positively adds to the solid gravity force. It is therefore concluded that as the solid loading increases the amount of solid entrained by the reversing gas at the central region below the cone deflector reduces. The solid concentration in the wall region massively increases while the core region remains relatively constant which can be seen in Fig. 24. The solid concentration and velocity profiles at the sample level, shown in Fig. 25 , indicate considerable hydrodynamic changes below the cone deflector as the solid loading increased. The gas velocity, however, shows exactly the opposite behavior with the axial velocity in the core region more than doubled when increasing the solid flow rare from 0.004 to $0.08 \mathrm{~kg} / \mathrm{s}$, while the velocity near the walls is slightly increased. Because the increase in the axial upward gas velocity in the centre takes place in a region that is at extremely low in solid concentration, the separator efficiency remains almost independent of the increase in solid loading. Accordingly, it is recommended to operate this downer reactor at a high solid flow rate for the following three main advantages:

i. Increased upward gas velocity towards the gas outlet pipe within the GDH region, therefore reducing the gas residence time in the reactor.

ii. Improved separator efficiency, as evident from the experimental and predicted results.

iii. Increasing the reactor processing capacity for biomass pyrolysis.

\subsubsection{Effect of the particle restitution and specularity coefficients}

It is understood that the particle size plays a major role on the separator efficiency such that the larger the particles size the higher the separator efficiency. Another important parameter of interest here is the degree of particle momentum loss or rebound upon hitting the solid surfaces, which is defined in the model through the restitution coefficient and specularity coefficient. The effective particle-wall restitution coefficient $\left(e_{s, w}\right)$ was determined experimentally by the co-authors from the ICFAR by measuring the rebound velocities of silica sand when hitting various types of solid 
surfaces; giving restitution coefficients ranging from 0.73 (Plexiglas surface) to 0.48 (paper surface). In this study, the same range of particle restitution coefficient was implemented in the model to investigate the effect of this parameter on the separator efficiency. The effect of the specularity coefficient $(\varphi)$ was investigated by using values of $\varphi=0,0.1$ and 1.0 , thus covering the two extreme ends of particle-wall interaction; free slip condition at $\varphi=0$ and no slip condition at $\varphi=1$. Reported studies (e.g. [25] ) have shown that the restitution coefficients have an effect on the solid velocity, gas velocity and solid concentration. It is also understood that as the restitution coefficient increases there is a corresponding increase in the wall shear stress. The specularity coefficient, on the other hand, has been reported to have a pronounced effect on the solid concentration, as increasing this parameter results in reducing the solid concentration at the wall.

Fig. 26 shows a comparison between the predicted and measured separator efficiency as a function of the particle restitution coefficient. It is clear that the predicted separator efficiency is a very weak function of this parameter. This is in good agreement with some of the reported literature (e.g. [25]) which suggest that the particle restitution coefficient (in the range 0.6 0.99) has limited effects on the solid velocity, gas velocity and the solid concentration in circulating fluidized bed reactors. The experimental data shows a slight decrease in the efficiency as the restitution coefficient decreases; however, this is still within a very limited range.

Fig. 27 shows the changes in the predicted separator efficiency with changing the value of the secularity coefficient. While there is clear drop in the efficiency as the specularity coefficient increases, this is still within a very limited range. This change is believed to be a result of the increase in the wall shear stress (no-slip condition), which in turn results in hindering the downward flow of the dense wall layer and hence giving rise to particle migration from the wall to the core followed by 
entrainment by the reversing gas towards the exit pipe. This phenomenon is demonstrated by the changes in the solid velocity and concentration profiles shown in Fig. 28. The specularity coefficient appears to have a significant effect on the gas velocity and solid concentration at the wall regions, which is in good agreement with the observation reported by Jin et al [25], and in spite of this there is a negligible effect on the separator efficiency.

\section{Conclusions}

The hydrodynamics in a downer pyrolysis reactor equipped with a novel gas-solid separator have been investigated theoretically using an Eulerian-Eulerian (two-fluid) CFD model. The novel separator, which consists of a cone deflector and a gas outlet pipe, was designed by the co-authors from the ICFAR (Canada). The model predictions were compared with experimental measurements of separator efficiency. This study revealed interesting hydrodynamic features around the cone deflector, where due to the restriction of the flow passage and solid deflection towards the walls, the region below the deflector in the downer reactor was completely transformed to behave like a riser, characterized by distinct upward gas flow at the core and dense falling solid layer at the walls. These distinct hydrodynamic features allowed for high efficiency of gas-solid separation up to $100 \%$. A new method for estimating the gas disengagement height $(\mathrm{GDH})$ was developed to help in estimating the gas residence time in this novel reactor. This study also included detailed sensitivity analysis of the separator efficiency towards the various operating conditions, including the effect of particle restitution and secularity coefficients. In the second part of this study, the present hydrodynamic model will be extended to include reaction kinetics and heat transfer to simulate the reactor thermochemical performance during the pyrolysis of biomass. 


\section{Notation}

$a$

A

B

C

$C_{D}$

$C_{\mu}, C_{1 \varepsilon}, C_{2 \varepsilon}$

$C_{f r, s_{i} s_{j}}$

$d_{S_{i}}$

$D_{C}$

$D_{g o}$

$e_{S_{i} s_{j}}$

$e_{s_{i}, w}$

$g$

$g_{0}$

$G_{k, g}$

$\overline{\bar{I}}$

$I_{2 D}$

$L_{1}, L_{2}$

$L_{S}$

$k_{g}$

$m_{\text {collected }}, m_{\text {in }}$

$\dot{m}_{g}, \dot{m}_{s}$

P
Gap between conical deflector and reactor wall (m)

Model parameter (-)

Model parameter (-)

Courant number (-)

Drag coefficient (-)

Constants (-)

Friction coefficient between solid phase $i$ and phase $j(-)$

Particle diameter of solid phase $i(\mathrm{~m})$

Reactor diameter $(\mathrm{m})$

Diameter of gas outlet pipe (m)

Particle-particle restitution coefficient (-)

Particle-wall restitution coefficient (-)

Gravity $\left(\mathrm{m} \mathrm{s}^{-2}\right)$

Radial distribution function (-)

Production of turbulent kinetic energy $\left(\mathrm{kg} \mathrm{m}^{-1} \mathrm{~s}^{-2}\right)$

Unit vector (-)

Second invariant of the deviatoric stress tensor $\left(\mathrm{s}^{-2}\right)$

Reactor dimension $(\mathrm{m})$

Separation length $(\mathrm{m})$

Turbulence kinetic energy $\left(\mathrm{m}^{2} \mathrm{~s}^{-2}\right)$

Mass of collected and fed solid particles respectively $(\mathrm{kg})$

Mass flow rate of gas and solid respectively $\left(\mathrm{kg} \mathrm{s}^{-1}\right)$

Pressure (pa) 


$\begin{array}{ll}\overline{\bar{S}} & \text { Strain rate }\left(\mathrm{s}^{-1}\right) \\ R e_{s_{i}} & \text { Reynolds number of solid phase } i(-) \\ t & \text { Time (s) } \\ \vec{u}_{g}, \vec{u}_{s_{i}} & \text { Gas and solid velocity vector }\left(\mathrm{m} \mathrm{s}^{-1}\right) \\ u_{s_{i}, w} & \text { Particle velocity at wall }\left(\mathrm{m} \mathrm{s}^{-1}\right) \\ v_{r, s_{i}} & \text { Terminal velocity correlation (-) } \\ X_{10}, X_{50}, X_{90} & \text { Particle size at accumulative volume fraction at } 10 \%, 50 \%, 90 \%\end{array}$

\section{Greek symbols}

$\alpha$

$\alpha_{g}, \alpha_{s_{i}}$

$\beta$

$\gamma_{\Theta_{s_{i}}}$

$\varepsilon_{g}$

$\eta$

$\Theta_{S_{i}}$

$\kappa_{\Theta_{s_{i}}}$

$\lambda_{s_{i}}$

$\mu_{l, g}, \mu_{t, g}$

$\mu_{s_{i}, \mathrm{col}}$

$\mu_{s_{i}, k i n}$

$\mu_{s_{i}, f r}$

$\Pi_{k, g}$

$\prod_{\varepsilon, g}$

$\rho_{s}, \rho_{g}$

$\overline{\bar{\tau}}$

$\sigma_{k}, \sigma_{\varepsilon}$

$\phi$

Angle of conical deflector (Degree)

Volume fraction of gas and solid phase $i$ respectively (-)

Momentum exchange coefficient $\left(\mathrm{kg} \mathrm{m}^{-3} \mathrm{~s}^{-1}\right)$

Collisional energy dissipation $\left(\mathrm{kg} \mathrm{m}^{-1} \mathrm{~s}^{-3}\right)$

Turbulent dissipation rate $\left(\mathrm{m}^{2} \mathrm{~s}^{-3}\right)$

Separation efficiency (-)

Granular temperature of solid phase $i\left(\mathrm{~m}^{2} \mathrm{~s}^{-2}\right)$

Diffusion coefficient of granular energy $\left(\mathrm{kg} \mathrm{m}^{-1} \mathrm{~s}^{-1}\right)$

Particle bulk viscosity $\left(\mathrm{kg} \mathrm{m}^{-1} \mathrm{~s}^{-1}\right)$

Viscosity of gas phase due to laminar, turbulent flow $\left(\mathrm{kg} \mathrm{m}^{-1} \mathrm{~s}^{-2}\right)$

Viscosity of solid phase $i$ due to collision $\left(\mathrm{kg} \mathrm{m}^{-1} \mathrm{~s}^{-1}\right)$

Viscosity of solid phase $i$ due to kinetics $\left(\mathrm{kg} \mathrm{m}^{-1} \mathrm{~s}^{-1}\right)$

Viscosity of solid phase $i$ due to friction $\left(\mathrm{kg} \mathrm{m}^{-1} \mathrm{~s}^{-1}\right)$

Influence of solid phases on gas phase $\left(\mathrm{m}^{2} \mathrm{~s}^{-3}\right)$

Influence of solid phases on gas phase $\left(\mathrm{m}^{2} \mathrm{~s}^{-4}\right)$

Solid and gas densities respectively $\left(\mathrm{kg} \mathrm{m}^{-3}\right)$

Shear stress tensor $\left(\mathrm{kg} \mathrm{m}^{-1} \mathrm{~s}^{-2}\right)$

Constants (-)

Angle of Internal friction (Degree) 
$\phi_{k s_{i}}$

$\varphi$

Energy exchange between phase $\mathrm{k}$ and solid phase $i\left(\mathrm{~kg} \mathrm{~m}^{-1} \mathrm{~s}^{-1}\right)$ Specularity coefficient (-)

Acknowledgement: The authors from the UK thank The Leverhulme Trust for a research grant (RPG-410).

\section{References}

[1] C. Mirgain, C. Briens, M. Del Pozo, R. Loutaty and M. Bergougnou, Modeling of feed vaporization in fluid catalytic cracking, Industrial \& Engineering Chemistry Research, 39 (2000) 4392-4399

[2] Y. Zhang, X. Wang, G. L. Riskowski, L. L. Christianson and S. E. Ford, Particle separation efficiency of a uniflow Deduster with different types of dusts, ASHRAE Transactions, 107 (2001) 93-98

[3] C. Huang, Z. Qian, M. H. Zhang and F. Wei, Solids mixing in a down-flow circulating fluidized bed of 0.418-m in diameter, Powder Technology, 161 (2006) 4852

[4] A. V. Bridgwater and G. V. C. Peacocke, Fast pyrolysis processes for biomass, Renewable \& Sustainable Energy Reviews, 4 (2000) 1-73

[5] E. Hoekstra, W. P. M. van Swaaij, S. R. A. Kersten and K. J. A. Hogendoorn, Fast pyrolysis in a novel wire-mesh reactor: Design and initial results, Chemical Engineering Journal, 191 (2012) 45-58

[6] M. I. Jahirul, M. G. Rasul, A. A. Chowdhury and N. Ashwath, Biofuels Production through Biomass Pyrolysis-A Technological Review, Energies, 5 (2012) 4952-5001 [7] M. Huard, C. Briens, F. Berruti and T. A. Gauthier, A Review of Rapid Gas-Solid Separation Techniques, International Journal of Chemical Reactor Engineering, 8 (2010)

[8] Y. Cheng, C. N. Wu, J. X. Zhu, F. Wei and Y. Jin, Downer reactor: From fundamental study to industrial application, Powder Technology, 183 (2008) 364-384 
[9] M. Huard, An investigation of a novel gas-solid separator for downer reactors, M.E.Sc. Thesis, The University of Western Ontario, London,Canada, (2009)

[10] M. Huard, F. Berruti and C. Briens, Experimental Study of a Novel Fast GasSolid Separator for Pyrolysis Reactors, International Journal of Chemical Reactor Engineering, 8 (2010)

[11] K. Ropelato, H. F. Meier and M. A. Cremasco, CFD study of gas-solid behavior in downer reactors: An Eulerian-Eulerian approach, Powder Technology, 154 (2005) 179-184

[12] Y. N. Kim, C. N. Wu and Y. Cheng, CFD simulation of hydrodynamics of gassolid multiphase flow in downer reactors: revisited, Chemical Engineering Science, 66 (2011) 5357-5365

[13] T. Samruamphianskun, P. Piumsomboon and B. Chalermsinsuwan, Computation of system turbulences and dispersion coefficients in circulating fluidized bed downer using CFD simulation, Chemical Engineering Research \& Design, 90 (2012) 21642178

[14] D. Winfield, M. Cross, N. Croft, D. Paddison and I. Craig, Performance comparison of a single and triple tangential inlet gas separation cyclone: A CFD Study, Powder Technology, 235 (2013) 520-531

[15] A. Kepa, Division of outlet flow in a cyclone vortex finder-The CFD calculations, Separation and Purification Technology, 75 (2010) 127-131

[16] K. W. Chu, B. Wang, D. L. Xu, Y. X. Chen and A. B. Yu, CFD-DEM simulation of the gas-solid flow in a cyclone separator, Chemical Engineering Science, 66 (2011) 834-847

[17] J. T. Jenkins and S. B. Savage, A Theory for the Rapid Flow of Identical, Smooth, Nearly Elastic, Spherical-Particles, Journal of Fluid Mechanics, 130 (1983) 187-202

[18] J. Ding and D. Gidaspow, A Bubbling Fluidization Model Using Kinetic-Theory of Granular Flow, Aiche Journal, 36 (1990) 523-538 
[19] S. M., The Particle-Particle Drag Term in a Multiparticle Model of Fluidization, National Technical Information Service, Springfield, VA. DOE/MC/21353-2373, NTIS/DE87006500, (1987)

[20] B. E. Launder and D. B. Spalding, Lectures in Mathematical Models of Turbulence, Academic press, London, (1972)

[21] S. A. VASQUEZ and V. A. IVANOV, A Phase Coupled Method for Solving Multiphase Problems on Unstructured Meshes, ASME 2000 Fluids Engineering Division Summer Meeting, (2000)

[22] R. Courant, K. Friedrichs and H. Lewy, On the partial difference equations of mathematical physics,, IBM Journal of Research and Development, 11 (1928) 215234

[23] P. C. Johnson and R. Jackson, Frictional-Collisional Constitutive Relations for Granular Materials, with Application to Plane Shearing, Journal of Fluid Mechanics, 176 (1987) 67-93

[24] D. Geldart, Y. Xue and H. Y. Xie, Measurement of bottom bed and transport disengaging heights in beds of fresh FCC catalyst, AIChE symposium series, 91 (1995) 93-101

[25] B. S. Jin, X. F. Wang, W. Q. Zhong, H. Tao, B. Ren and R. Xiao, Modeling on High-Flux Circulating Fluidized Bed with Geldart Group B Particles by Kinetic Theory of Granular Flow, Energy \& Fuels, 24 (2010) 3159-3172 


\section{List of tables}

Table 1. Dimensions of the downer reactor and the cone separator

\begin{tabular}{clcl}
\hline parameters & Value & parameters & Value \\
\hline $\mathrm{L}_{1}[\mathrm{~cm}]$ & 98.6 & $\mathrm{D}_{\mathrm{c}}[\mathrm{cm}]$ & 7 \\
$\mathrm{~L}_{2}[\mathrm{~cm}]$ & 34.9 & $\mathrm{~L}_{\mathrm{s}}[\mathrm{cm}]$ & $0,3.5,7$ \\
a [cm] & 0.35 & $\mathrm{D}_{\mathrm{go}}[\mathrm{cm}]$ & 0.95 \\
$\alpha$ [Degree] & $60,90,120$ & & \\
\hline
\end{tabular}

Table 2. Constitutive relations for the gas-solid flow

$$
\begin{aligned}
& \text { solids pressure } \\
& P_{s_{i}}=\alpha_{s_{i}} \rho_{s_{i}} \Theta_{s_{i}}+\sum_{j=1, j \neq i}^{3} \frac{\left(d_{s_{i}}+d_{s_{j}}\right)^{3}}{4 d_{s_{i}}^{3}} g_{0, s_{i} s_{j}} \rho_{s_{i}} \alpha_{s_{i}} \alpha_{s_{j}} \Theta_{s_{i}}\left(1+e_{s_{i} s_{j}}\right) \quad(T 1-1)
\end{aligned}
$$

solids shear viscosity

$$
\mu_{s_{i}}=\mu_{s_{i}, \text { col }}+\mu_{s_{i}, k i n}+\mu_{s_{i}, f r}
$$

Collisional viscosity [21]

$$
\mu_{s_{i}, \text { col }}=\frac{4}{5} \alpha_{s_{i}} \rho_{s_{i}} d_{s_{i}} g_{0, s_{i} s_{i}}\left(e_{s_{i} s_{i}}+1\right)\left(\frac{\Theta_{s_{i}}}{\pi}\right)^{1 / 2}
$$

Kinetic viscosity [22]

$\mu_{s_{i}, k i n}=\frac{\alpha_{s_{i}} \rho_{s_{i}} d_{s_{i}} \sqrt{\Theta_{s_{i}} \pi}}{6\left(3-e_{s_{i} s_{i}}\right)}\left[1+\frac{2}{5}\left(e_{s_{i} s_{i}}+1\right)\left(3 e_{s_{i} s_{i}}-1\right) \alpha_{s_{i}} g_{0, s_{i} s_{i}}\right]$

Friction viscosity [23]

$$
\mu_{s_{i}, f r}=\frac{P_{s_{i}} \sin \phi}{2 \sqrt{I_{2 D}}}
$$

Bulk viscosity [24]

$$
\lambda_{s_{i}}=\frac{4}{3} \alpha_{s_{i}} \rho_{s_{i}} d_{s_{i}} g_{0, s_{i} s_{i}}\left(e_{s_{i} s_{i}}+1\right)\left(\frac{\Theta_{s_{i}}}{\pi}\right)^{1 / 2}
$$

Radial distribution function

$$
\begin{gathered}
g_{0, s_{i} s_{i}}=\left(1-\left(\frac{\alpha_{s}}{\alpha_{s, \max }}\right)^{1 / 3}\right)^{-1}+\frac{1}{2} d_{s_{i}} \sum_{j=1}^{3} \frac{\alpha_{s_{j}}}{d_{s_{j}}} \\
g_{0, s_{i} s_{j}}=\frac{d_{s_{j}} g_{0, s_{i} s_{i}}+d_{s_{i}} g_{0, s_{j} s_{j}}}{d_{s_{i}}+d_{s_{j}}}
\end{gathered}
$$


$\alpha_{s}=\sum_{j=1}^{3} \alpha_{s_{j}}$

Gas viscosity

$$
\mu_{g}=\mu_{l, g}+\mu_{t, g}, \quad \mu_{t, g}=C_{\mu} \alpha_{g} \rho_{g} \frac{k_{g}^{2}}{\varepsilon_{g}}
$$

Gas-solid drag coefficient [25]

$\beta_{g s_{i}}=\frac{3 \rho_{g} \alpha_{s_{i}} \alpha_{g}}{4 u_{r, s_{i}}^{2} d_{s_{i}}} C_{D}\left(\frac{R e_{s_{i}}}{v_{r, s_{i}}}\right)\left|\vec{u}_{g}-\vec{u}_{s_{i}}\right|$

$v_{r, s_{i}}=0.5\left(A-0.06 R e_{s_{i}}+\sqrt{\left(0.06 R e_{s_{i}}\right)^{2}+0.12 R e_{s_{i}}(2 B-A)+A^{2}}\right)$

$A=\alpha_{g}^{4.14}, \quad\left\{\begin{array}{c}B=0.8 \alpha_{g}^{1.28}\left(\alpha_{g} \leq 0.85\right) \\ B=\alpha_{g}^{2.65}\left(\alpha_{g}>0.85\right)\end{array}\right.$

$C_{D}=\left(0.63+\frac{4.8}{\sqrt{\operatorname{Re}_{s_{i}} / v_{r, s_{i}}}}\right)^{2}$

$R e_{s_{i}}=\frac{d_{s_{i}} \rho_{g}\left|\vec{u}_{g}-\vec{u}_{s_{i}}\right|}{\mu_{g}}$

Solid-solid drag coefficient [19]

$\beta_{s_{i} s_{j}}=\frac{3\left(1+e_{s_{i} s_{j}}\right)\left(\frac{\pi}{2}+C_{f r, s_{i} s_{j}} \frac{\pi^{2}}{8}\right) \alpha_{s_{i}} \rho_{s} \alpha_{s_{j}} \rho_{s}\left(d_{s_{i}}+d_{s_{j}}\right)^{2} g_{0, s_{i} s_{j}}}{2 \pi \rho_{s}\left(d_{s_{i}}^{3}+d_{s_{j}}^{3}\right)}\left|\vec{u}_{s_{i}}-\vec{u}_{s_{j}}\right|$

Diffusion coefficient of granular energy [21]

$\kappa_{\Theta_{s_{i}}}=\frac{150 \rho_{s} d_{s_{i}}\left(\pi \Theta_{s_{i}}\right)^{\frac{1}{2}}}{384\left(e_{s_{i} s_{i}}+1\right) g_{0, s_{i} s_{i}}}\left[1+\frac{6}{5} \alpha_{s_{i}} g_{0, s_{i} s_{i}}\left(e_{s_{i} s_{i}}+1\right)\right]^{2}$

$$
+2 \alpha_{s_{i}}^{2} \rho_{s} d_{s_{i}} g_{0, s_{i} s_{i}}\left(e_{s_{i} s_{i}}+1\right)\left(\frac{\Theta_{s_{i}}}{\pi}\right)^{\frac{1}{2}}(T 1-13)
$$

Collisional energy dissipation [24],

$\gamma_{\Theta_{s_{i}}}=\frac{12\left(1-e_{S_{i} s_{i}}^{2}\right) g_{0, s_{i} s_{i}}}{d_{s_{i}} \pi^{1 / 2}} \alpha_{s_{i}}^{2} \rho_{s} \Theta_{s_{i}}{ }^{3 / 2}$

Table 3. Particle size distribution employed in the CFD model

\begin{tabular}{c|c|c|c}
\hline & Solid Phase I & Solid Phase II & Solid Phase III \\
\hline Particle size $(\mu \mathrm{m})$ & $X_{10}=124$ & $X_{50}=206$ & $X_{90}=328$ \\
Volume fraction (\%) & $20 \%$ & $60 \%$ & $20 \%$ \\
\hline
\end{tabular}


Table 4. Gas and solid phase boundary/operating conditions used in the CFD model

\begin{tabular}{l|c|c}
\hline Boundary/operating condition & Experiment & Model \\
\hline Gas mass flow rate, $\dot{m}_{g}[\mathrm{~kg} / \mathrm{s}]$ & $0.0039,0.0239$, & $0.0039^{*}, 0.0239$, \\
Gas density, $\rho_{g}\left[\mathrm{~kg} / \mathrm{m}^{3}\right]$ & 0.0439 & 0.0439 \\
Gas viscosity & 1.2 & $1.2^{*}$ \\
{$[\mathrm{~kg} /(\mathrm{m} . \mathrm{s})]$} & $1.8 \times 10^{-5}$ & $1.8 \times 10^{-5^{*}}$ \\
Gas outlet pressure, $P_{g o}[\mathrm{~Pa}]$ & 0 (ambient) & $0^{*}$ (ambient) \\
Particle density, $\rho_{P}\left[\mathrm{~kg} / \mathrm{m}^{3}\right]$ & 2650 & $2650^{*}$ \\
Solid mass flow rate, $\dot{m}_{s}[\mathrm{~kg} / \mathrm{s}]$ & $0.017,0.083$ & $0.004^{*}, 0.02,0.04,0.08$ \\
Mean particle size, $\bar{d}_{s}[\mu \mathrm{m}]$ & 188 & Mixture $(124,206$, \\
Angle of cone deflector $[\mathrm{degree}]$ & $60,90,120$ & $328)$ \\
Separation length, $L_{S}[\mathrm{~m}]$ & 0 & $60^{*}, 90,120$ \\
\hline
\end{tabular}

"Default simulation conditions, unless otherwise specified 


\section{List of figures}

Figure 1. Demonstration of biomass pyrolysis in various dual fluidized bed reactor configurations.

Figure 1. Schematic diagram of the experimental apparatus

Figure 3. Illustration of experimental (a) cold model downer setup and (b) gas-solids separation zone.

Figure 4. Particle size distribution of silica sand used in the experiment

Figure 5. Computational domain and meshing in a cross-sectional view.

Figure 6. Example of the gas-solid flow structure in the overall downer column and around the separation device. (a) Gas phase velocity vectors, with the color code restricted to a maximum of $5 \mathrm{~m} / \mathrm{s}$ to allow visualization (b) Solid phase volume fraction in a section, with the color code restricted to a maximum of $4 \times 10^{-4}$ to allow visualization.

Figure 7. Example of the radial profiles of the (a) axial gas velocity and (b) solid volume fraction. The data was taken at the sample level of $3.9 \mathrm{~cm}$ below the gas exit tip.

Figure 8. Sectional view of the solid and gas axial velocity vectors demonstrating the various characteristic flow zones in the downer reactor and around the separation device. To allow visualization of the low ranges, the magnitude of the velocity vector was restricted to a maximum of $5 \mathrm{~m} / \mathrm{s}$. The color code bar indicates the range of gas/solid velocity.

Figure 9. Illustration of the method used to determine the GDH demonstrated in typical results obtained at the default model operating conditions (a) pressure gradient method (b) axial gas velocity method. 
Figure 10. The effect of operation contitions on the gas disengagement height. The default simulation conditions (Table 4) were used in all simulations, unless otherwise specified.

Figure 11. Overall sensitivity analysis of the effect the of operating conditions on the separator efficicny. The default simulation conditions (Table 4) were used in all simulations, unless otherwise specified.

Figure 12. Effect of the separation length on the separator efficiency. The simulation was carried out using the default operating conditions.

Figure 13. Gas velocity vectors at various normalized separation lengths. To allow visualization of the low ranges, the magnitude of the velocity vector was restricted to a maximum of $5 \mathrm{~m} / \mathrm{s}$. The color code bar indicates the range of gas velocity.

Figure 14. Effect of the separation length on the (a) radial profiles of axial gas velocity and (b) solid volume fraction (for the selected particle size $d_{s}=124 \mu \mathrm{m}$ ). The data was taken at the sample level of $3.9 \mathrm{~cm}$ below the gas exit pipe tip.

Figure 15. Effect of the cone deflector angle on the separator efficiency. The experiment data was obtained from Hurad et al. [10] The simulation was carried out using the default operating conditions.

Figure 16. Gas velocity vectors at various cone deflector angles. To allow visualization of the low ranges, the magnitude of the velocity vector was restricted to a maximum of $5 \mathrm{~m} / \mathrm{s}$. The color code bar indicates the range of gas velocity.

Figure 17. Solid volume fraction for the particle size of $d_{s}=324 \mu \mathrm{m}$ at two different deflector angles. To allow visualization of the low ranges, the solid volume fraction was restricted to $5 \times 10^{-4}$. The color code bar indicates the range of solid volume fraction.

Figure 18. Effect of the gas mass flow rate on the separator efficiency. The simulation was carried out at the default operating conditions. 
Figure 19. Gas velocity vectors at various gas flow rates. The color code bars indicate the range of gas velocity.

Figure 20. Solid volume fraction at various gas mass flow rate for the particle size $d_{s}=206 \mu \mathrm{m}$. To allow visualization of the low ranges, the magnitude of the solid volume fraction is restricted here to a maximum of $2 \times 10^{-4}$. The color code bar indicates the range of the solid volume fraction.

Figure 21. Effect of the gas flow rate on the (a) radial profiles of axial gas velocity and (b) solid volume fraction (for the selected particle size $d_{s}=206 \mu \mathrm{m}$ ). The data was taken at the sample level of $3.9 \mathrm{~cm}$ below the gas exit pipe tip.

Figure 22. Effect of the solid loading on the separator efficiency. The simulation was carried out at the default operating conditions.

Figure 23. Gas velocity vectors at various solid flow rates. To allow visualization of the low ranges, the magnitude of the velocity vector was restricted to a maximum of $5 \mathrm{~m} / \mathrm{s}$. The color code bar indicates the range of gas velocity.

Figure 24. Solid volume fraction at various solid flowrates for the selected particle size $d_{s}=206 \mu \mathrm{m}$. The color code bars indicate the range of solid volume concentration.

Figure 25. Effect of the solid mass flow rate on (a) radial profiles of axial gas velocity and (b) solid volume fraction (for the selected particle size $d_{s}=206 \mu \mathrm{m}$ ). The data was taken at the sample level of $3.9 \mathrm{~cm}$ below the gas exit pipe tip.

Figure 26. Effect of the particle-wall restitution coefficient $\left(e_{s, w}\right)$ on the separator efficiency. The simulation was carried out at the default operating conditions.

Figure 27 . Effect of specularity coefficient on the separator efficiency. The simulation was carried out at the default operating conditions. 
Figure 28. Effect of the specularity coefficient on the (a) radial profiles of axial gas velocity and (b) solid volume fraction (for the selected particle size $d_{s}=206 \mu \mathrm{m}$ ). The data was taken at the sample level of $3.9 \mathrm{~cm}$ below the tip of the gas exit pipe. 
Fig.1

Click here to download high resolution image

flue gas

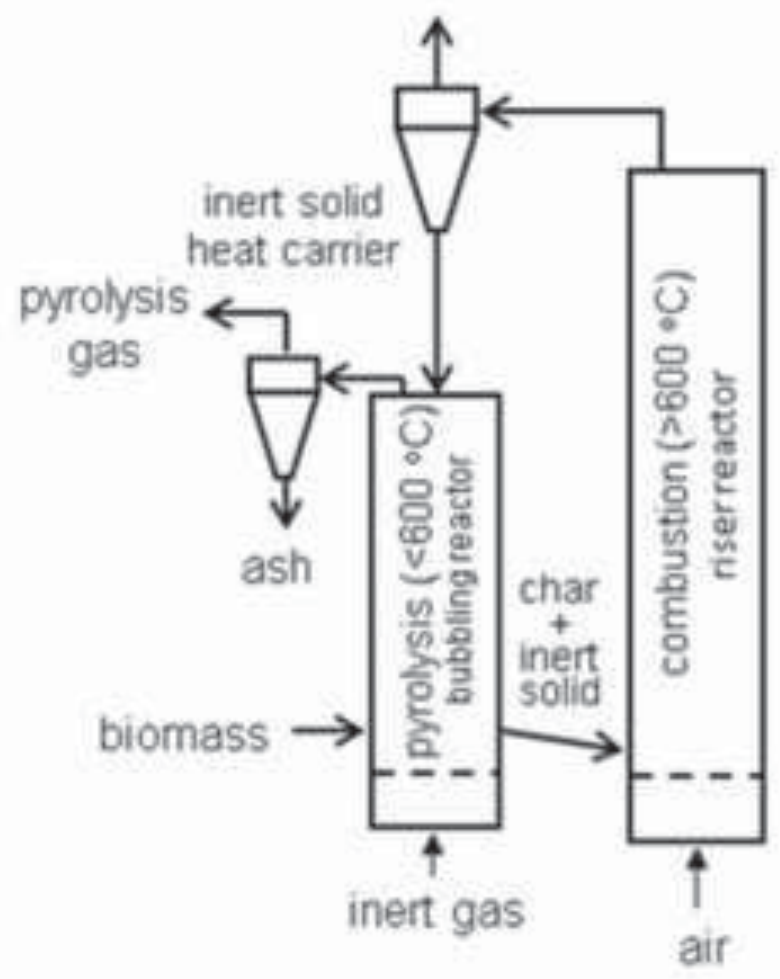

(a) Bubbling pyrolysis reactor flue gas

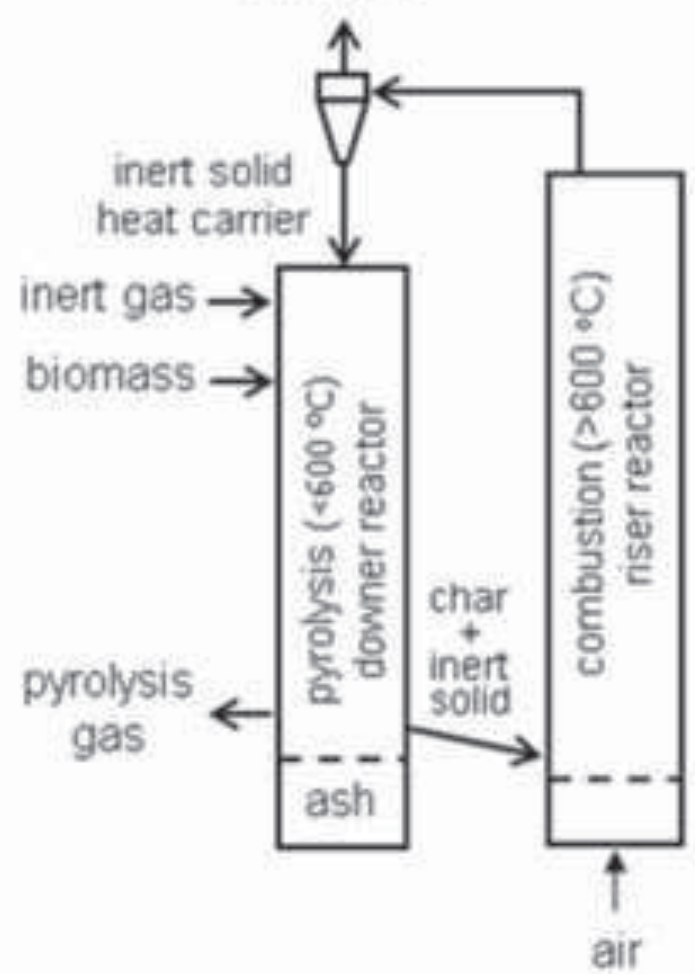

(b) Downer pyrolysis reactor

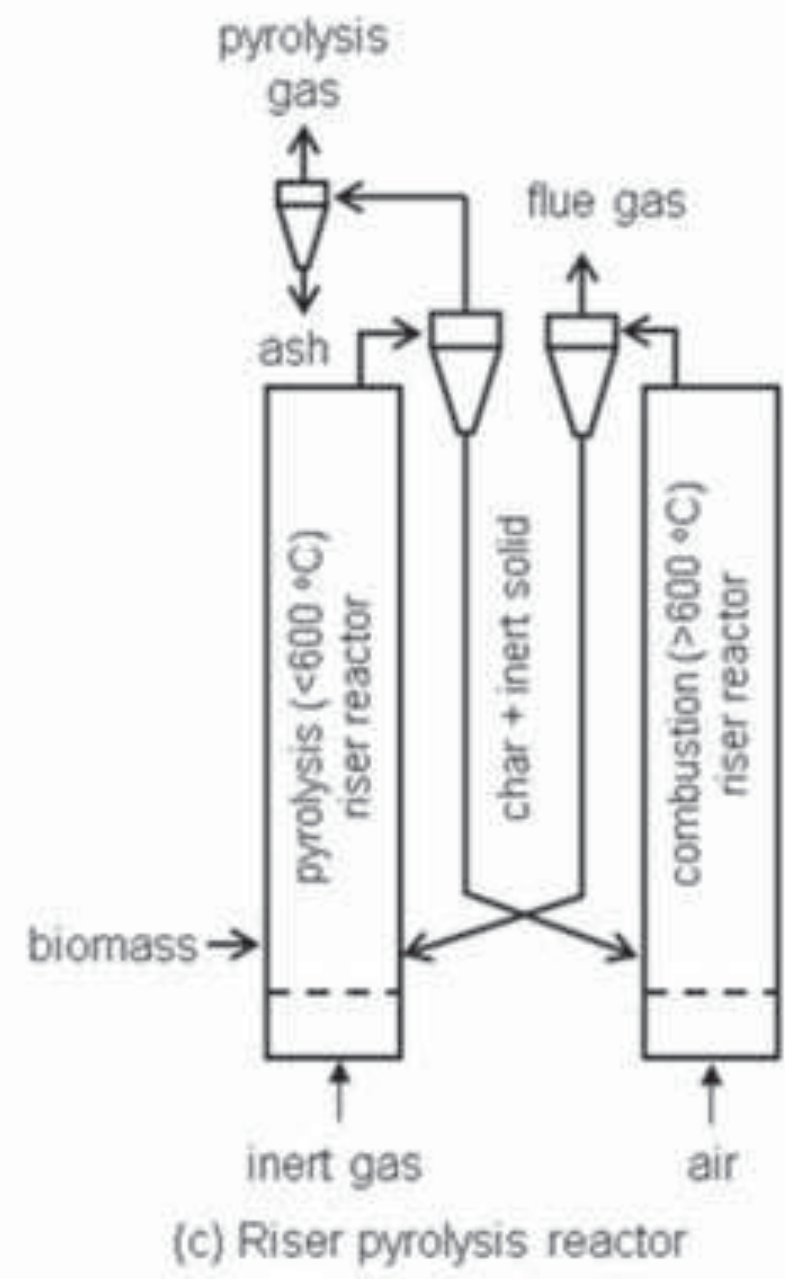




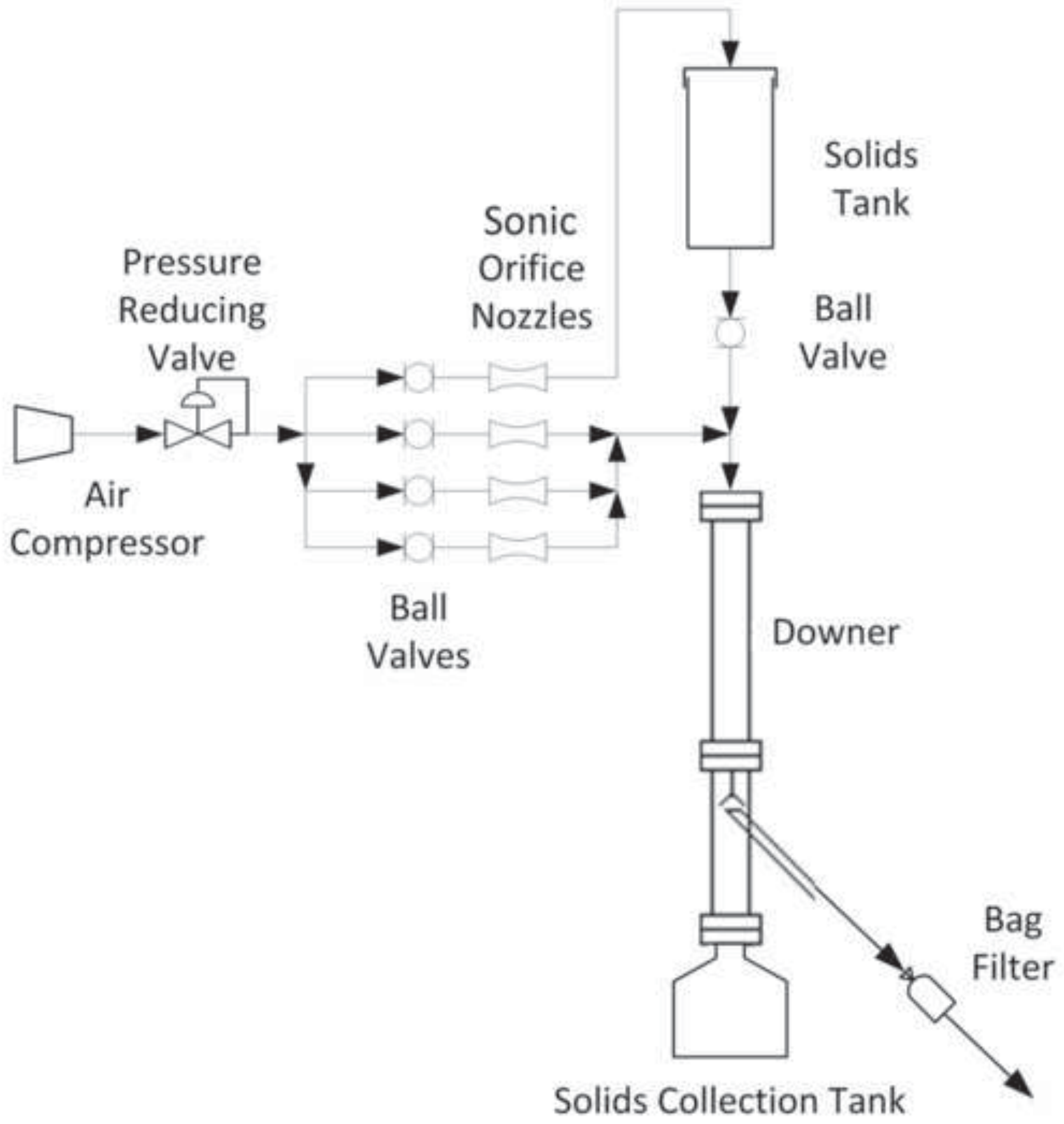



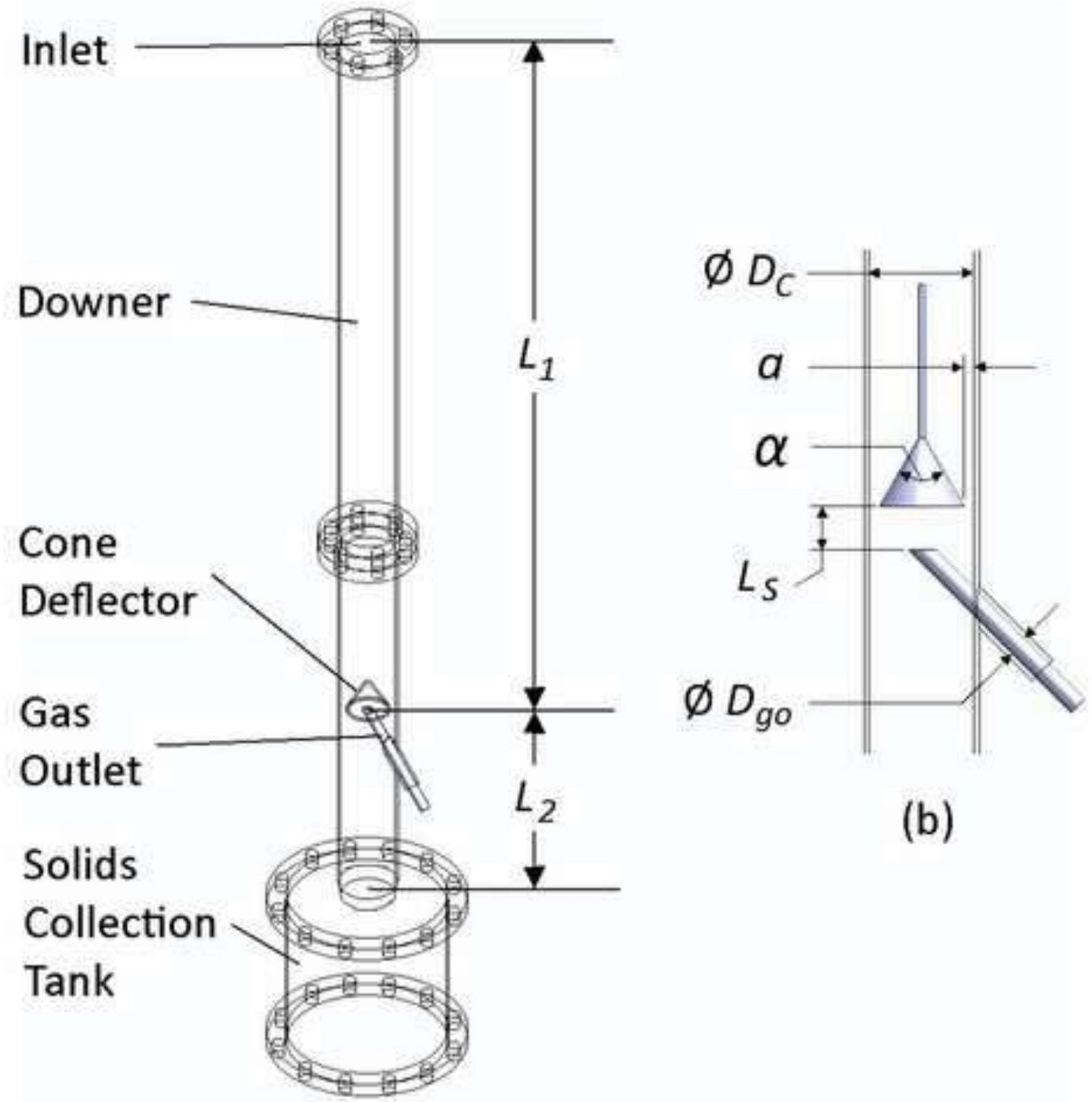

(b)

(a) 
Fig.4

Click here to download high resolution image

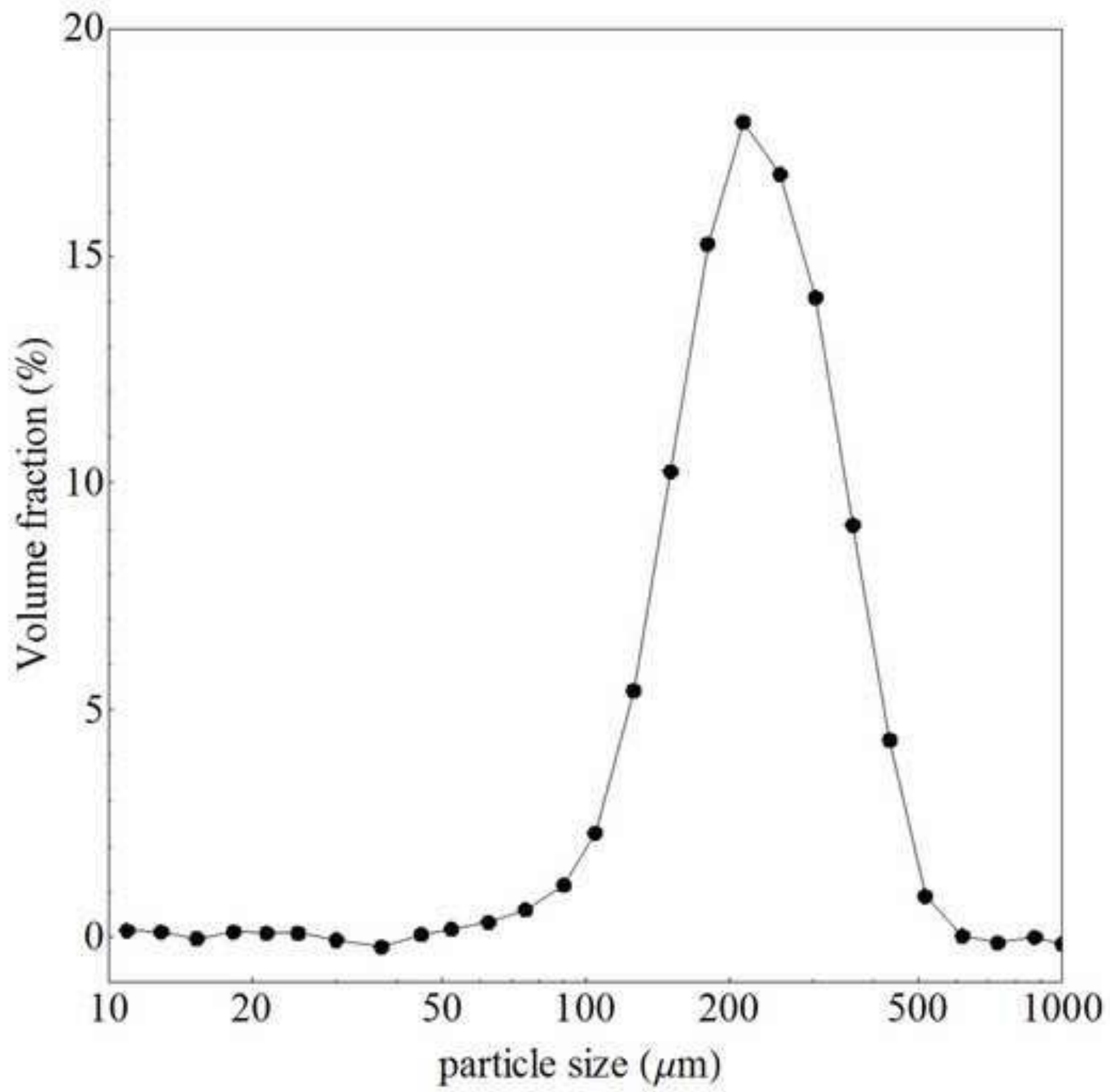


Fig.5

Click here to download high resolution image
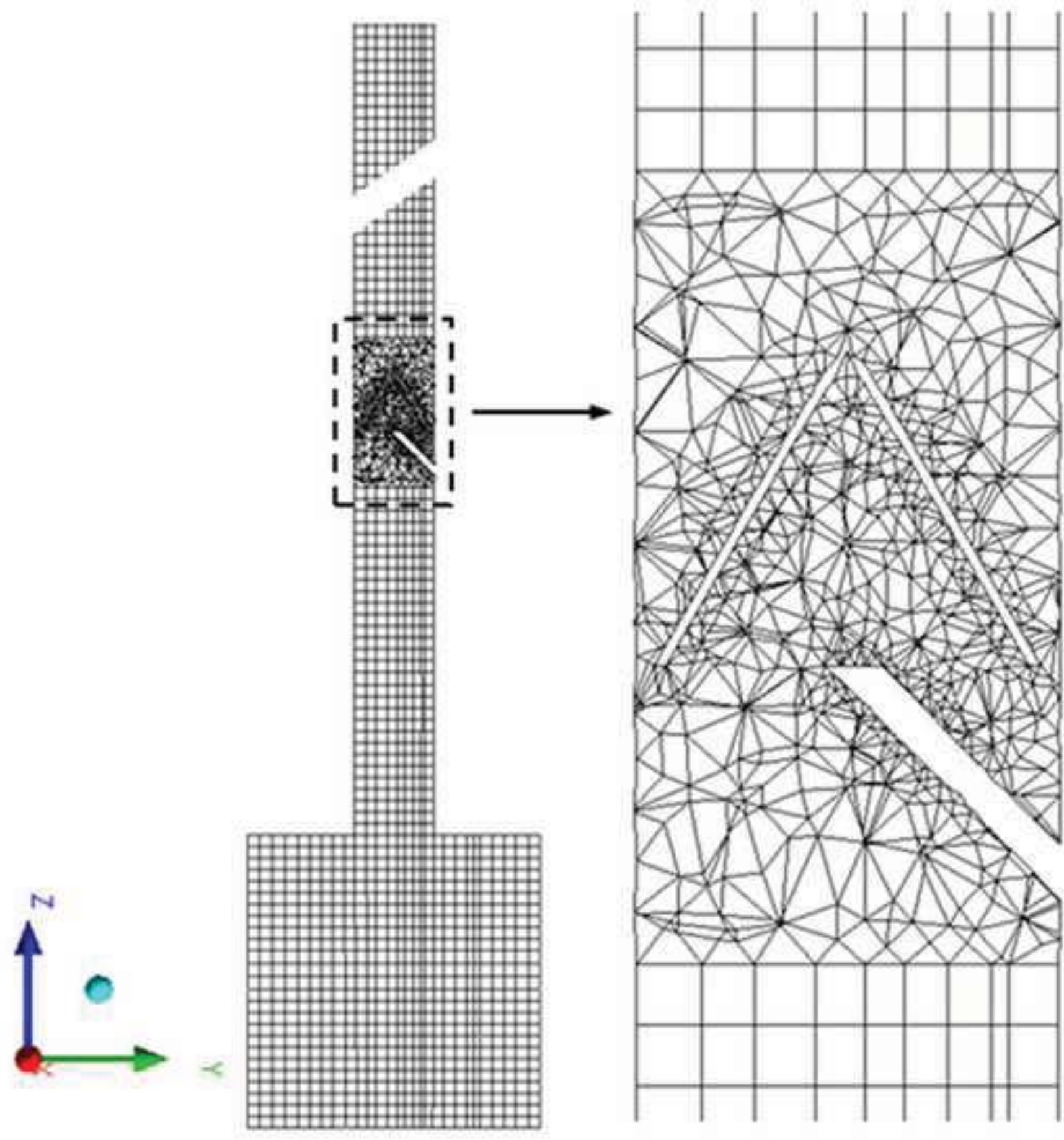


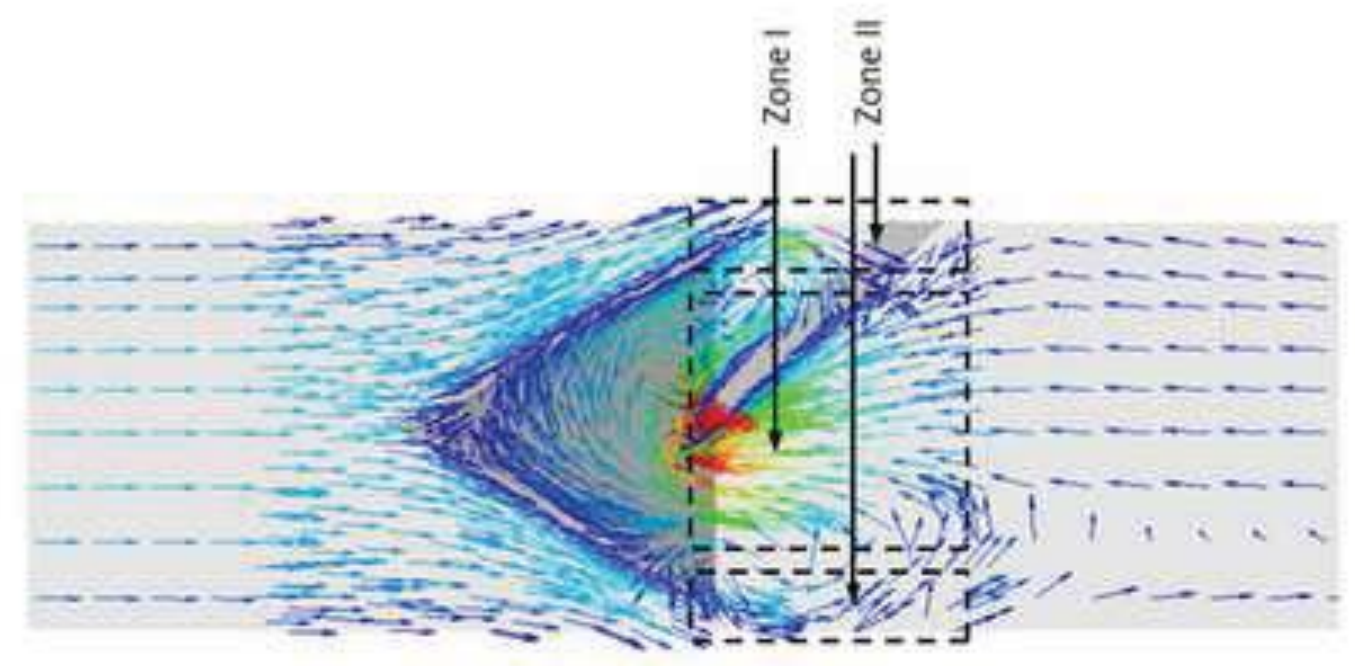

요






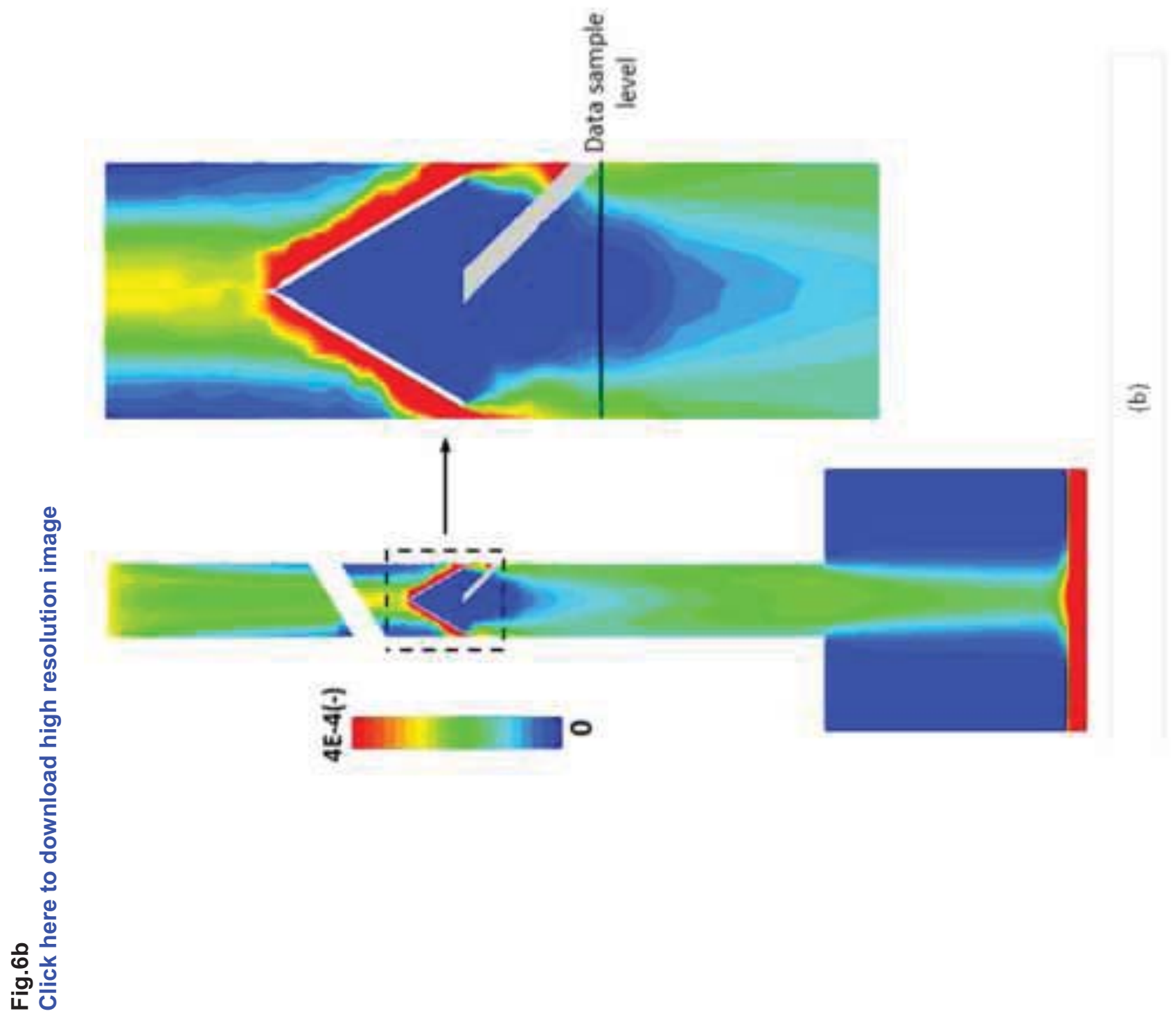




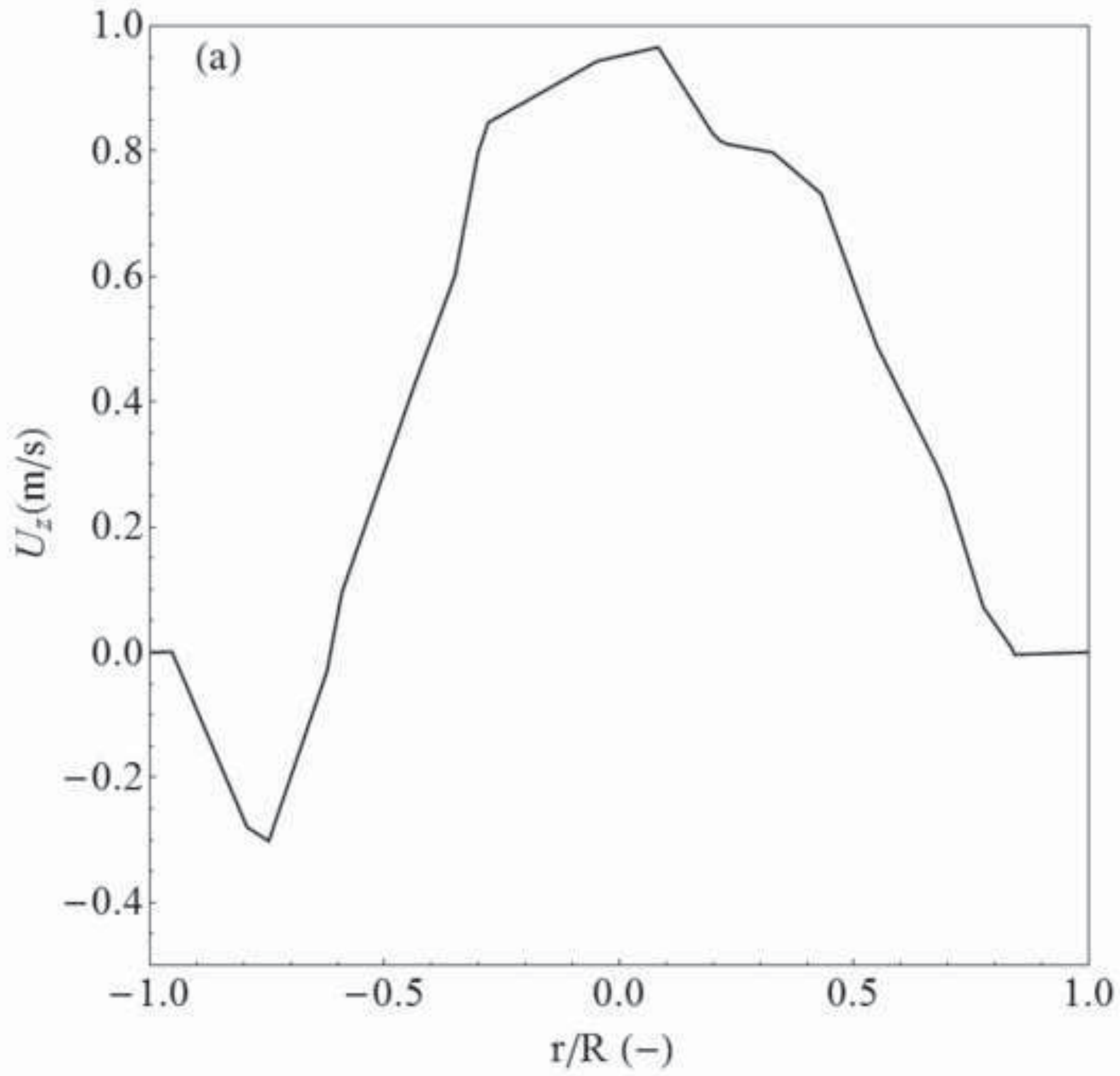




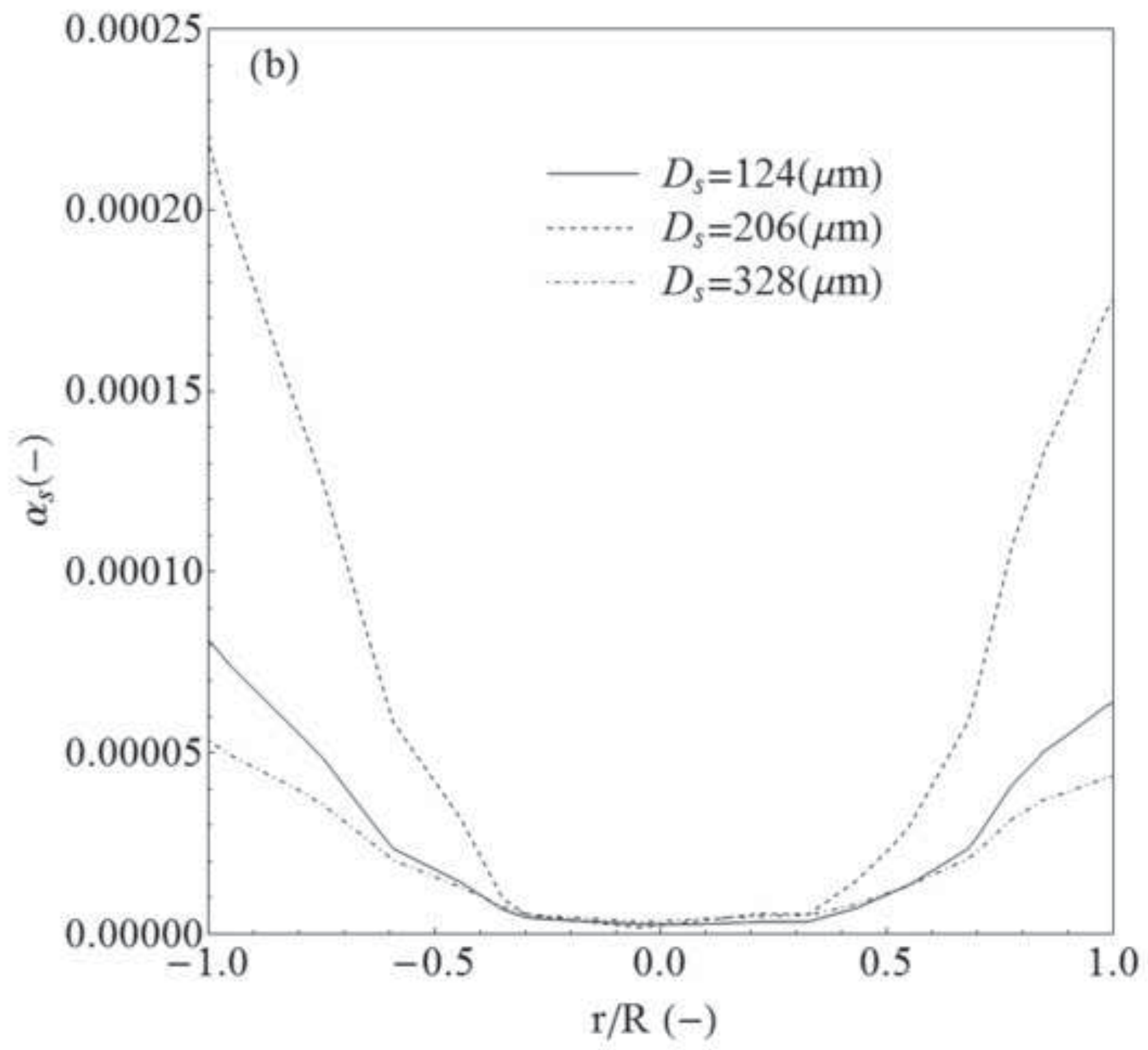




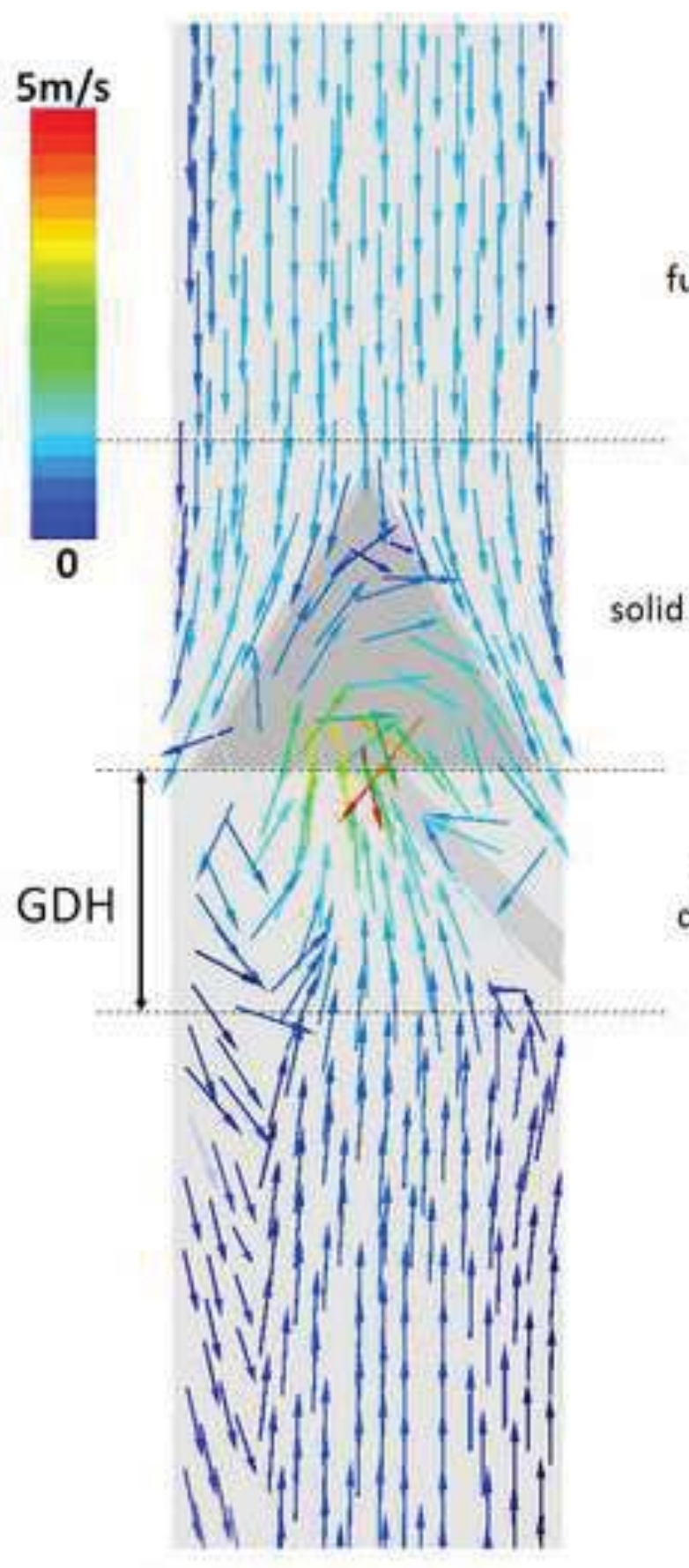

(a) Gas velocity vector
Zone I

fully developed flow

Zone II

olid deflection and gas removal

Zone III gas disengagement


(b) Solid velocity vector 


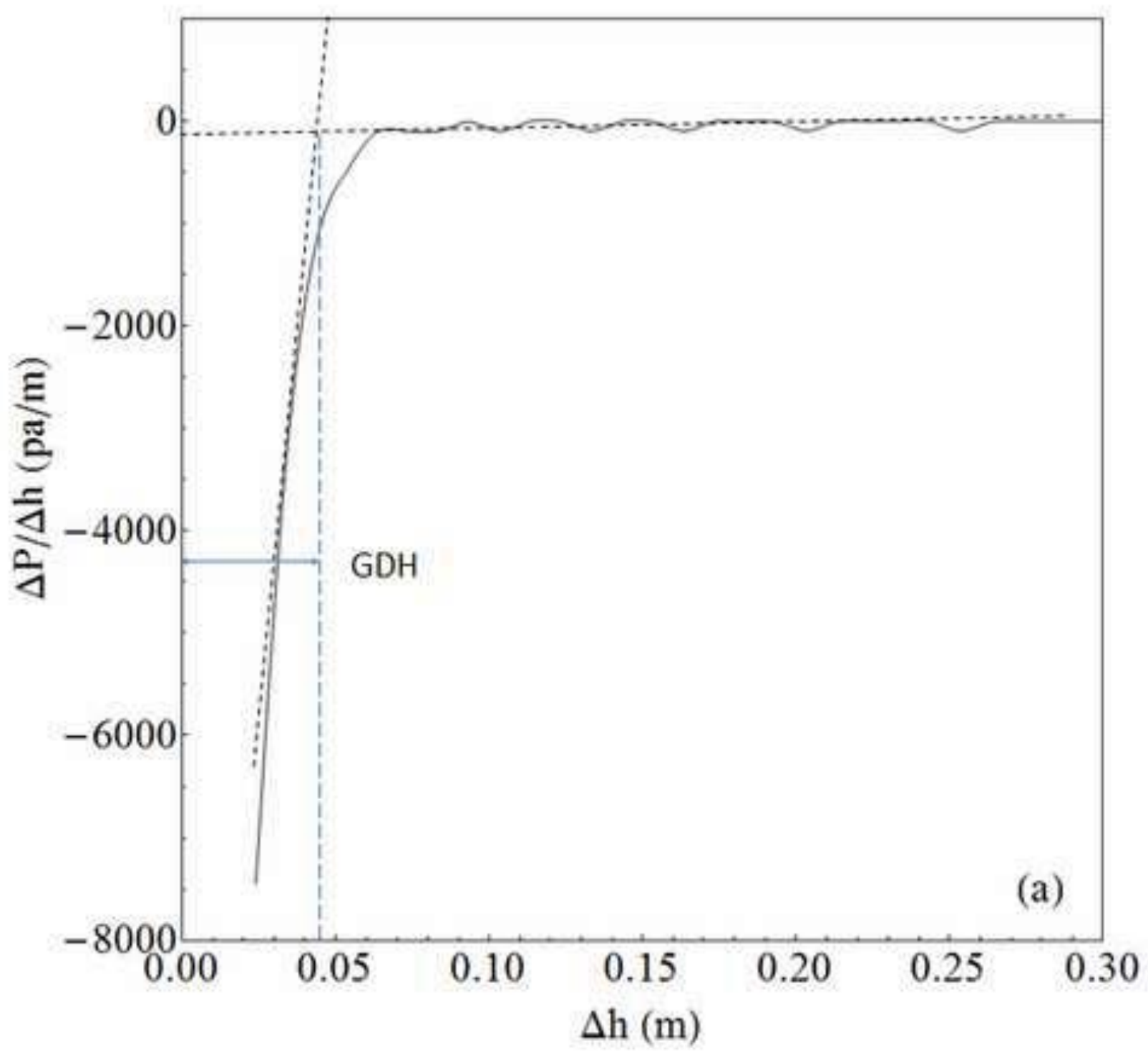




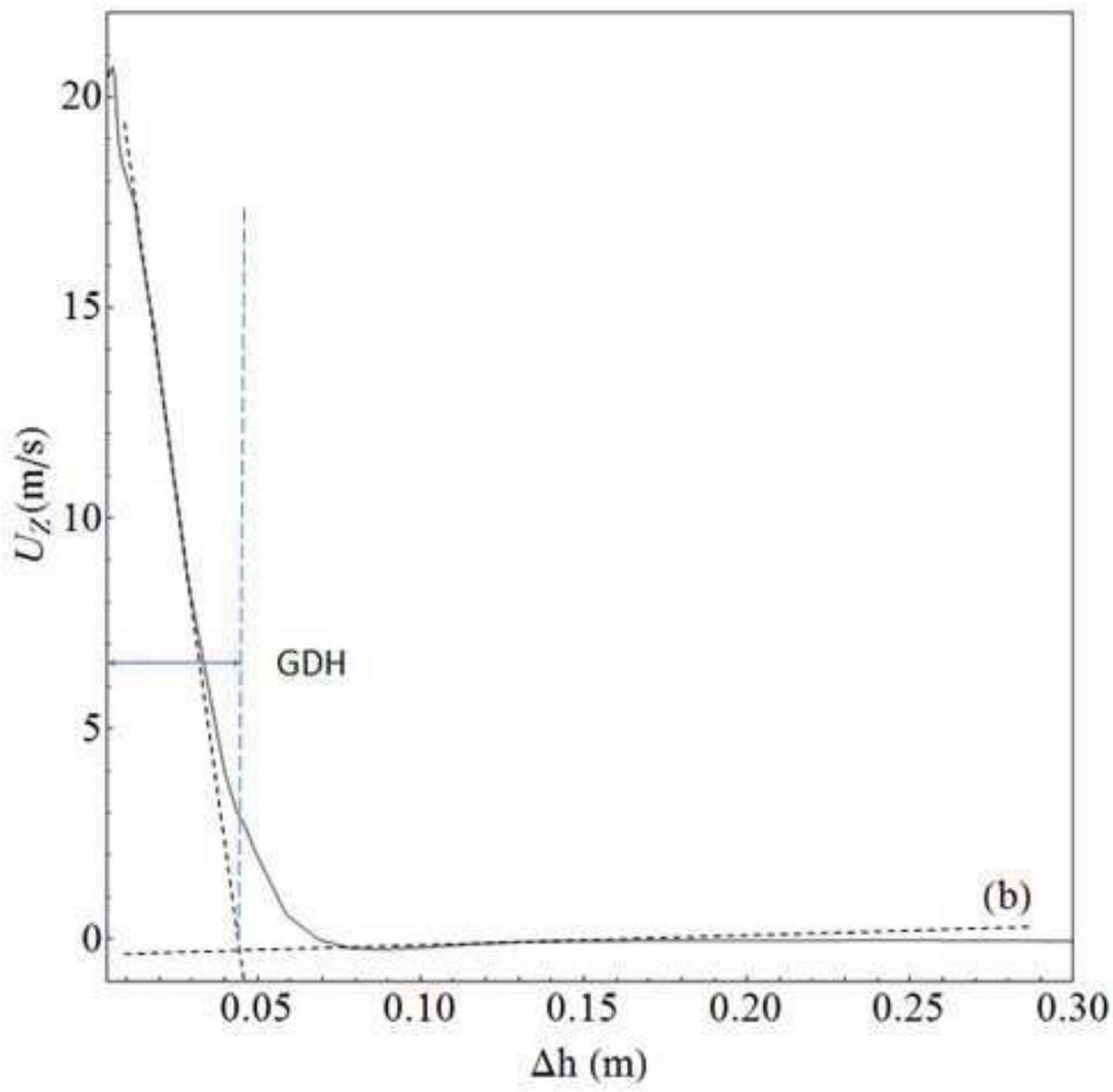





s/8ม6ع20.0 $={ }^{6} u$

s/8ํ6ع00 $0={ }^{B} u \varphi$


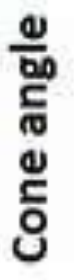

$\omega_{0} L={ }^{s} 7$

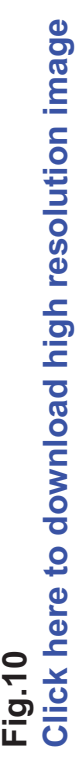

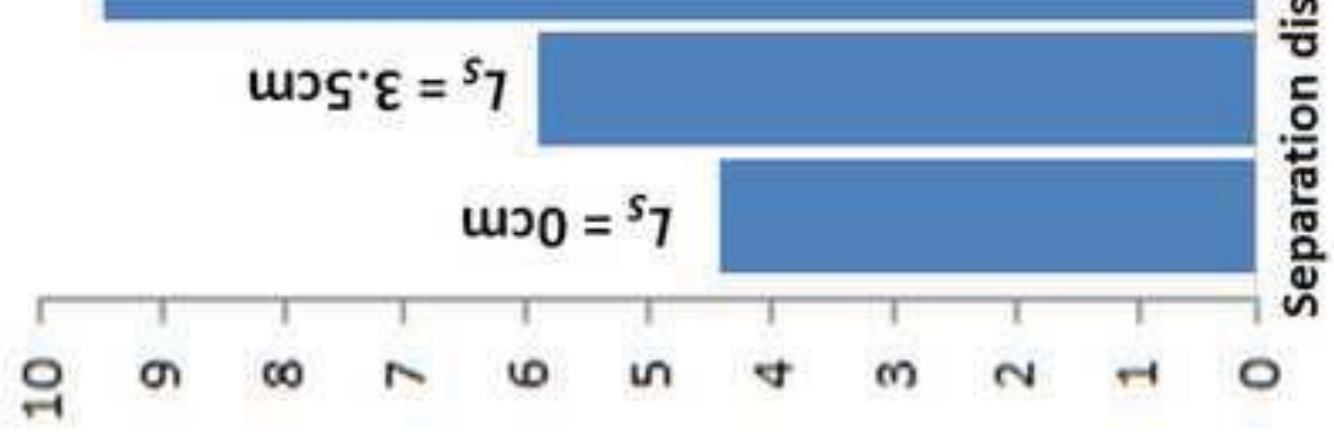

$$
\text { (แว) }{ }^{s} \text { T+HOS }
$$




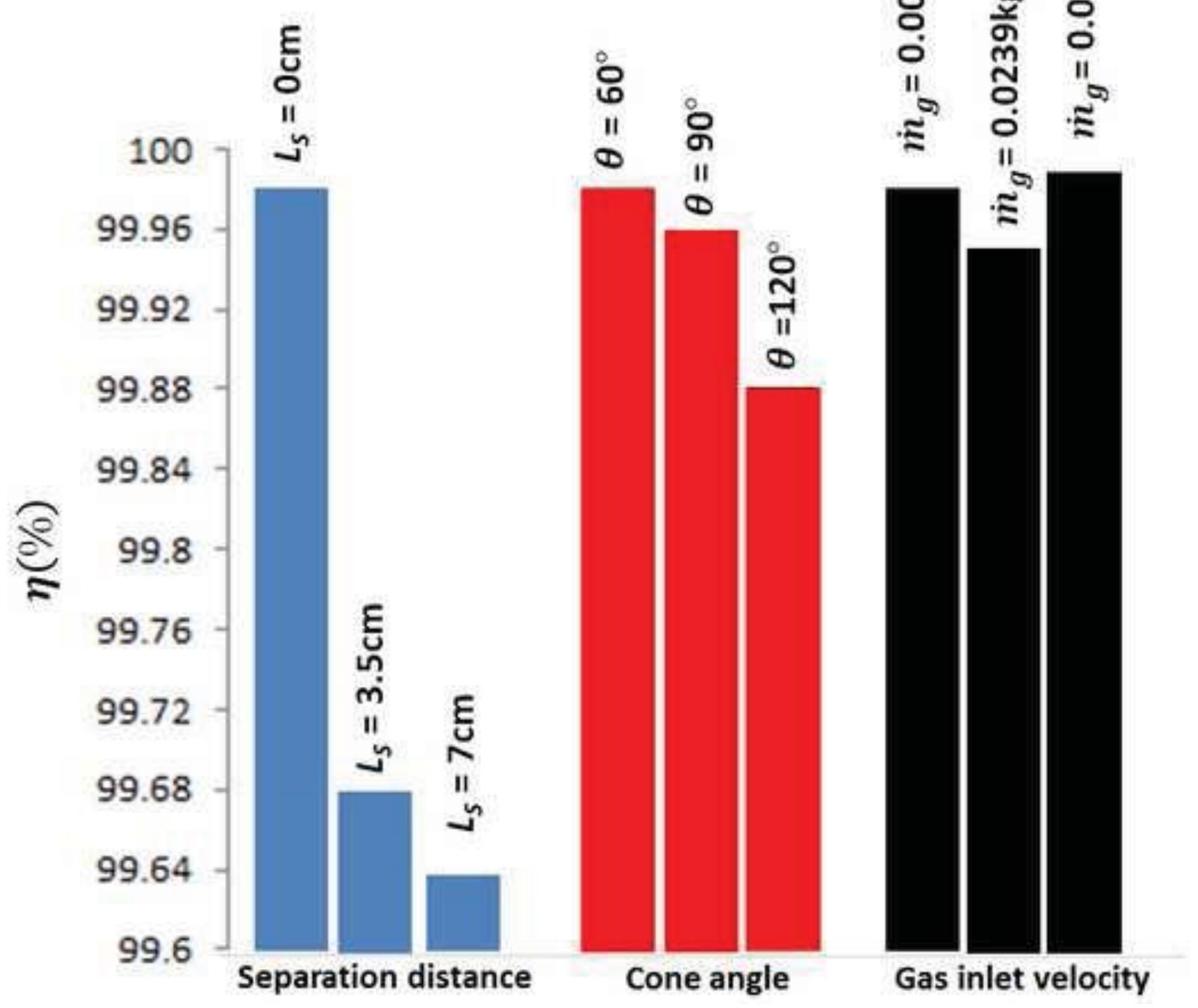



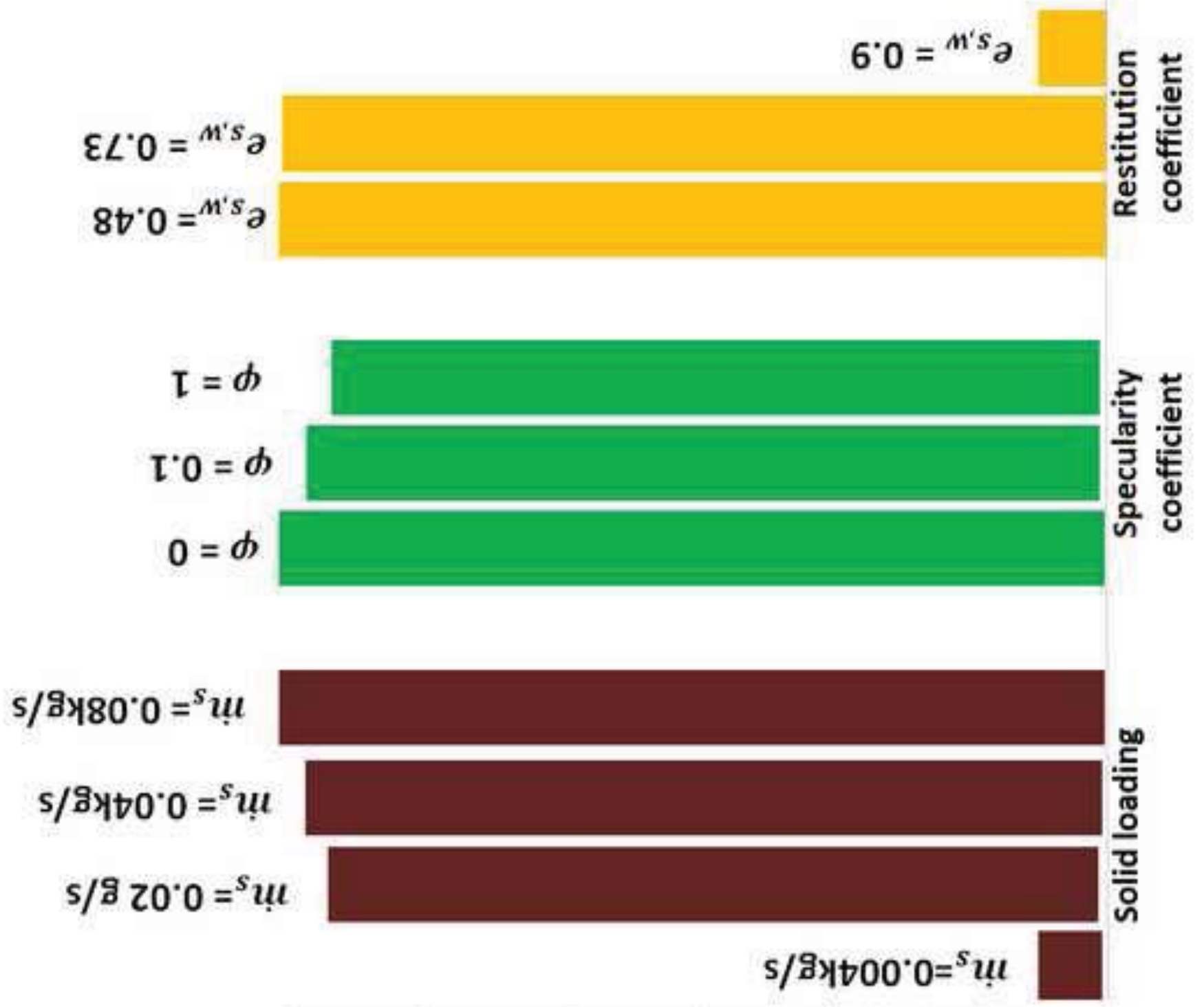



\begin{tabular}{|c|c|c|c|c|c|c|}
\hline$r$ & $T$ & $T$ & $T$ & $T$ & $T$ & ᄀ \\
\hline \multirow[t]{3}{*}{$\underset{r}{8}$} & $\stackrel{\text { in }}{\sigma}$ & $\begin{array}{l}\text { g } \\
\text { gn }\end{array}$ & $\stackrel{\text { ก }}{\text { ğ }}$ & $\begin{array}{l}\sigma \\
\sigma \\
\sigma\end{array}$ & $\stackrel{n}{\infty}$ & $\begin{array}{l}\text { n̊ } \\
\text { ద }\end{array}$ \\
\hline & कृ & gi & कุ & बे & ọ & gi \\
\hline & $\begin{array}{l}\sigma \\
\text { बे }\end{array}$ & бे & $\begin{array}{l}\sigma \\
\sigma\end{array}$ & & $\underset{\sigma}{\sigma}$ & గi \\
\hline
\end{tabular}

(\%) $u$ 


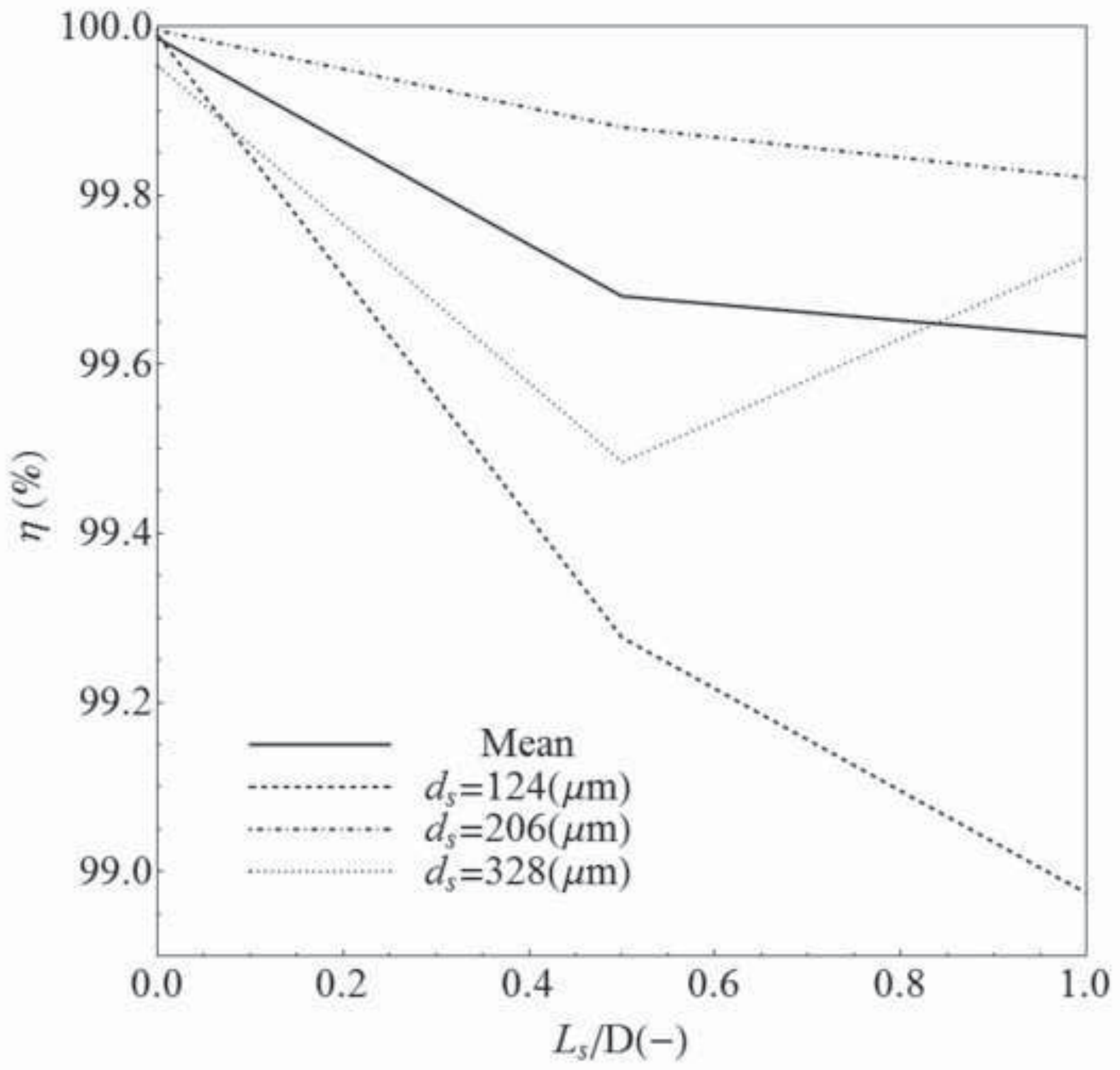



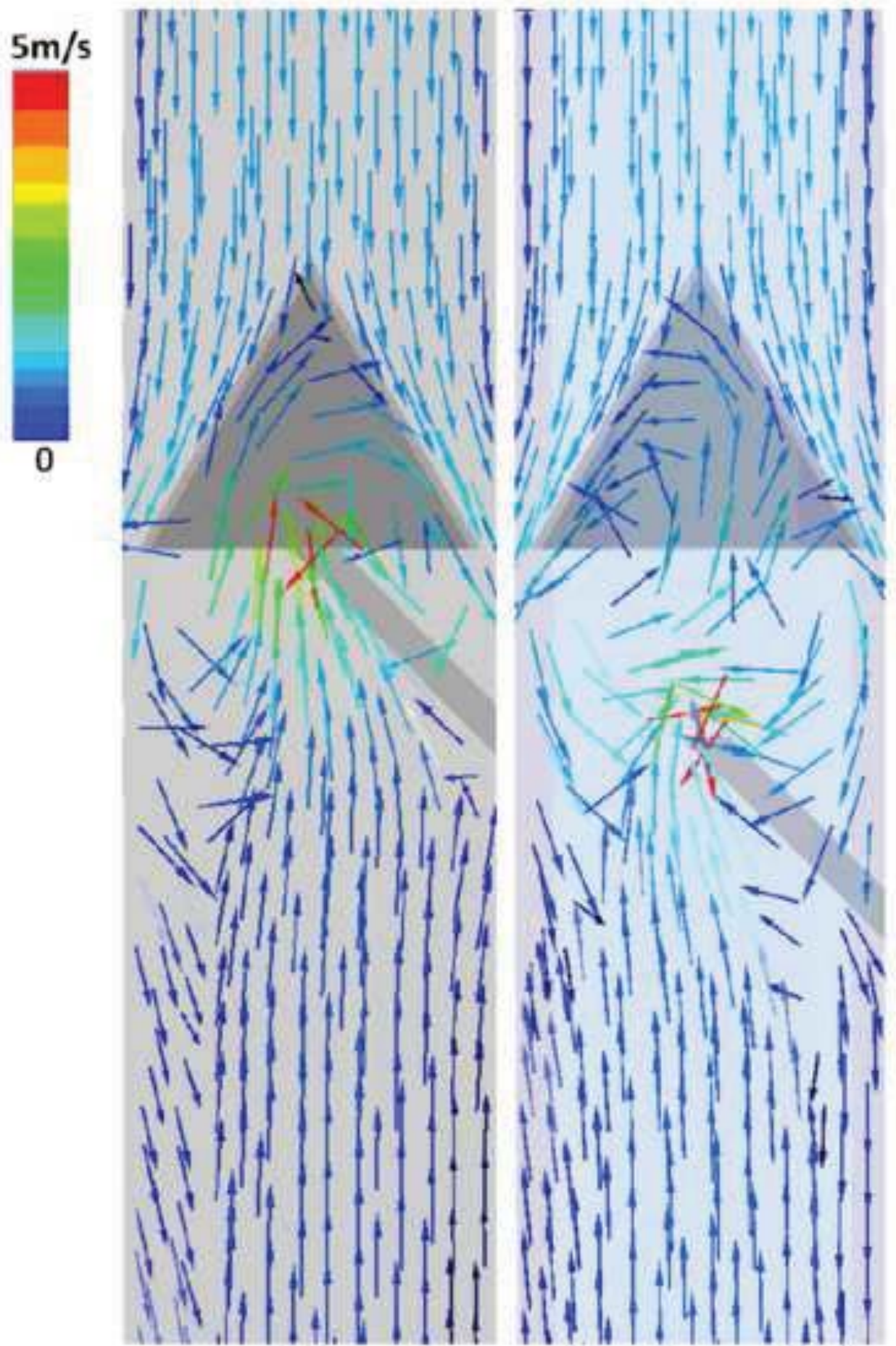

$$
L_{s} / D=0
$$

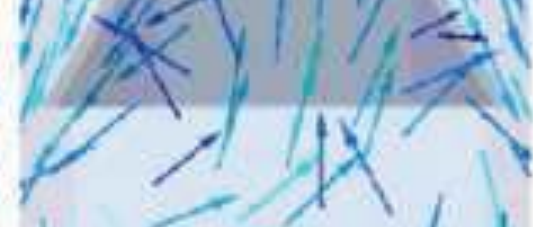

$$
L_{s} / D=0.5
$$

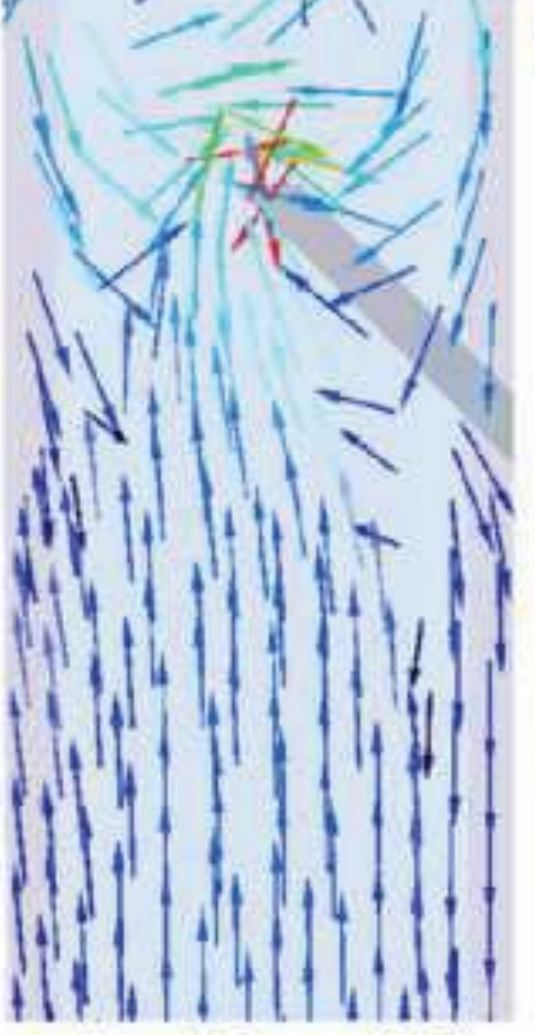

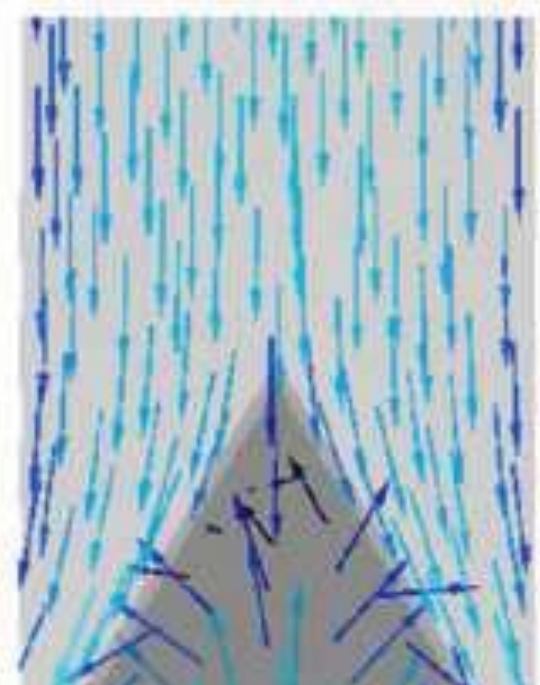
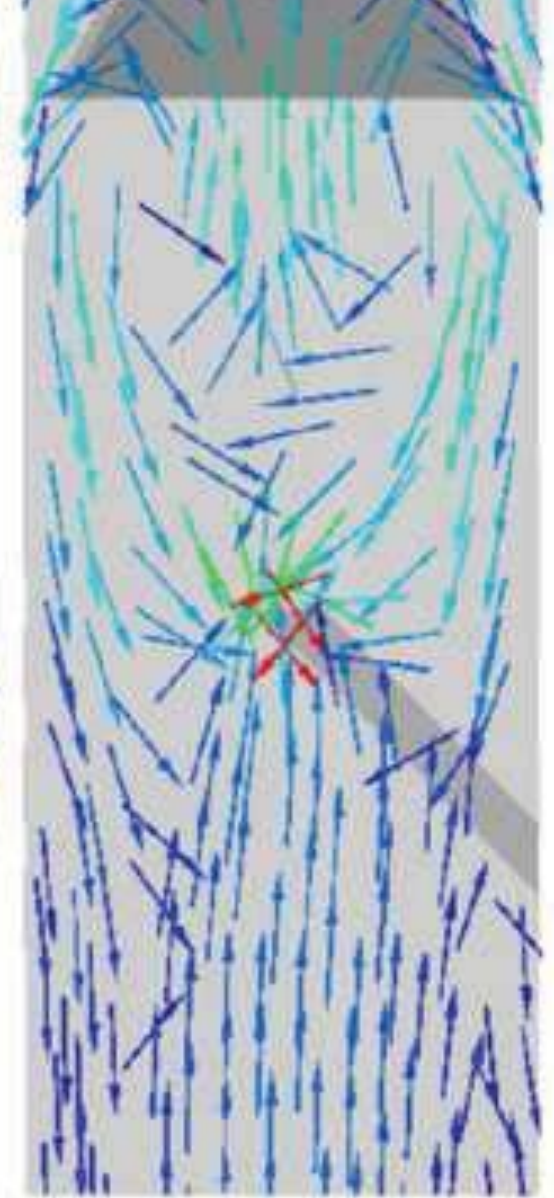

$$
L_{s} / D=1
$$




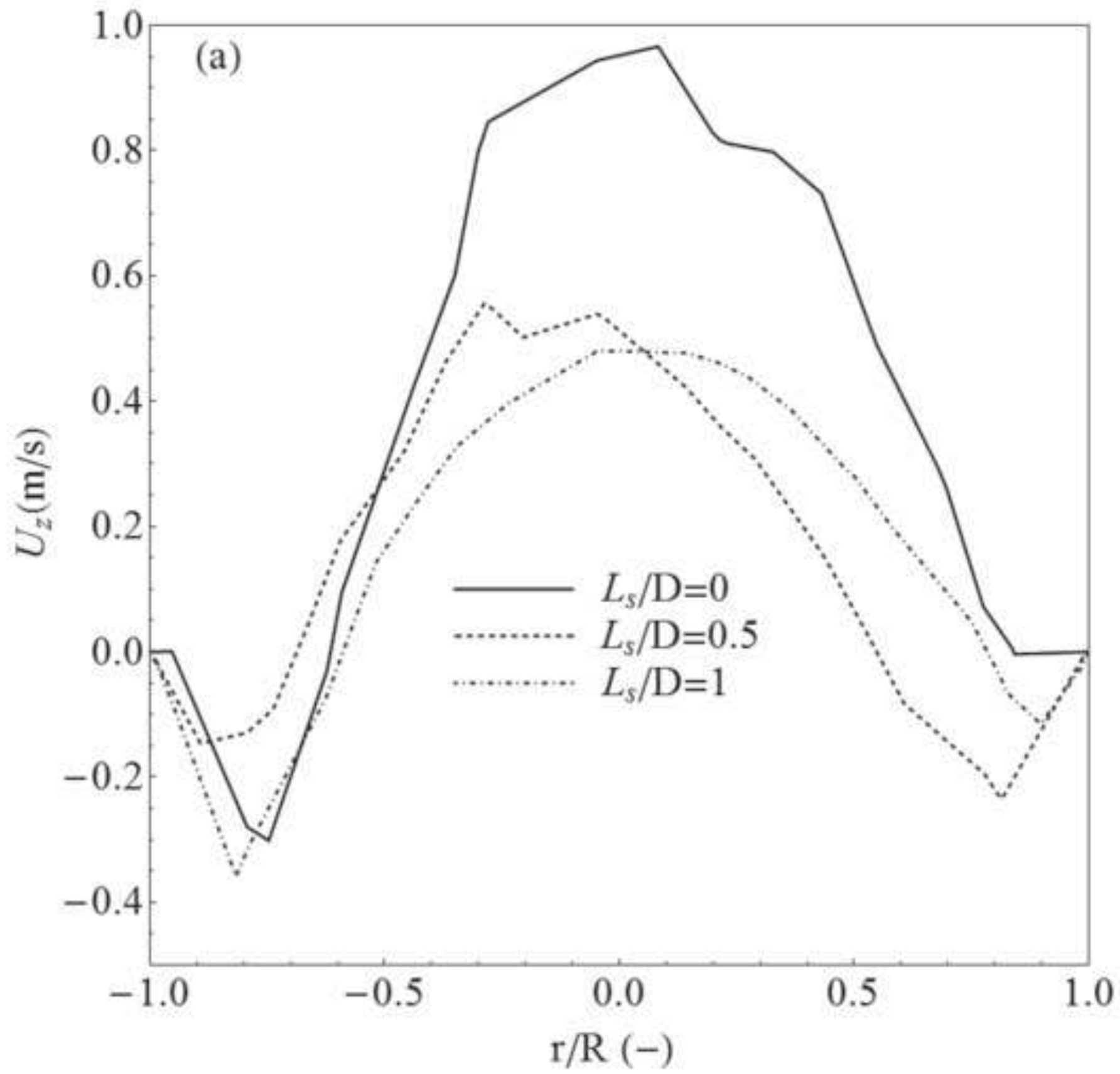




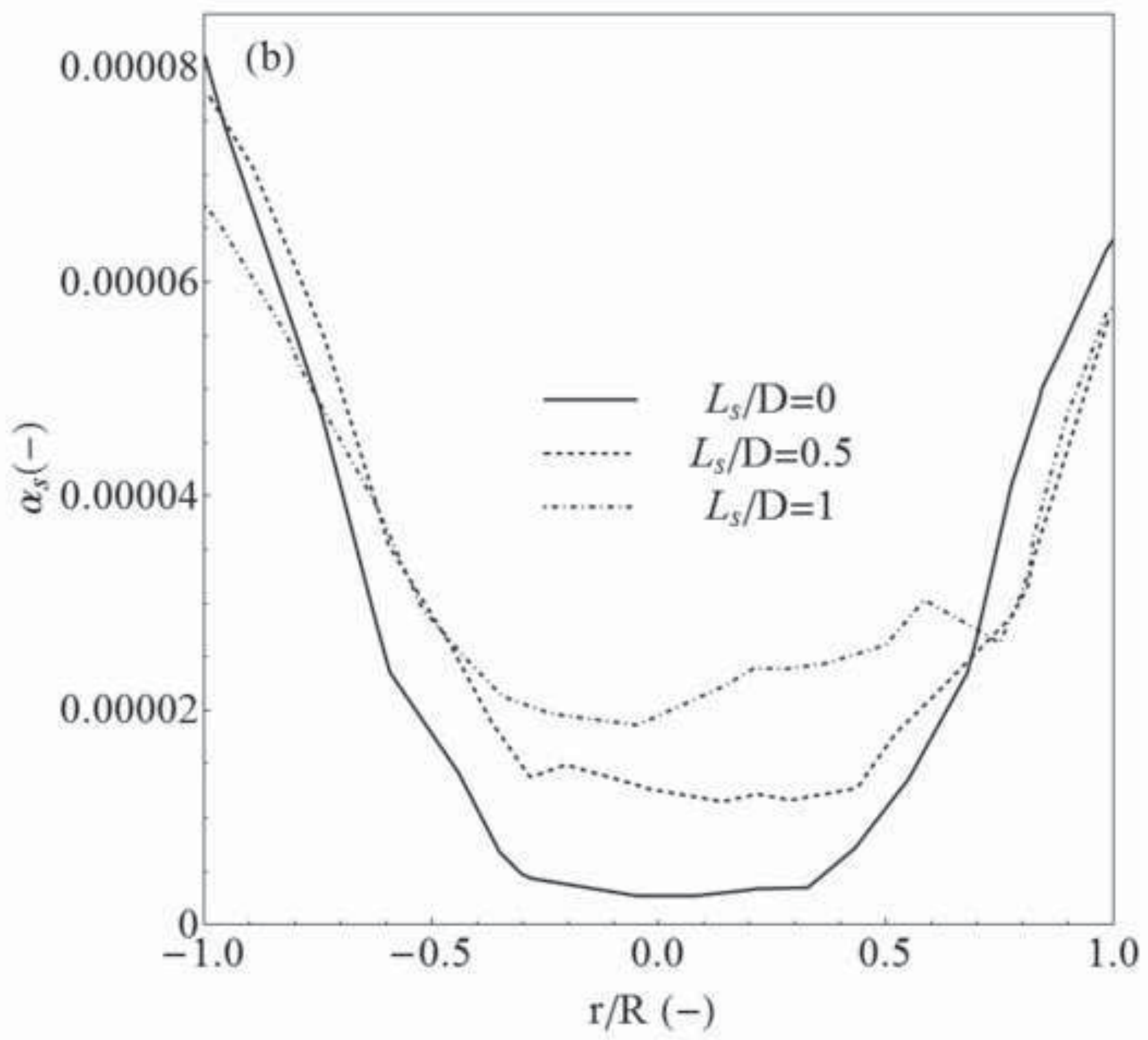




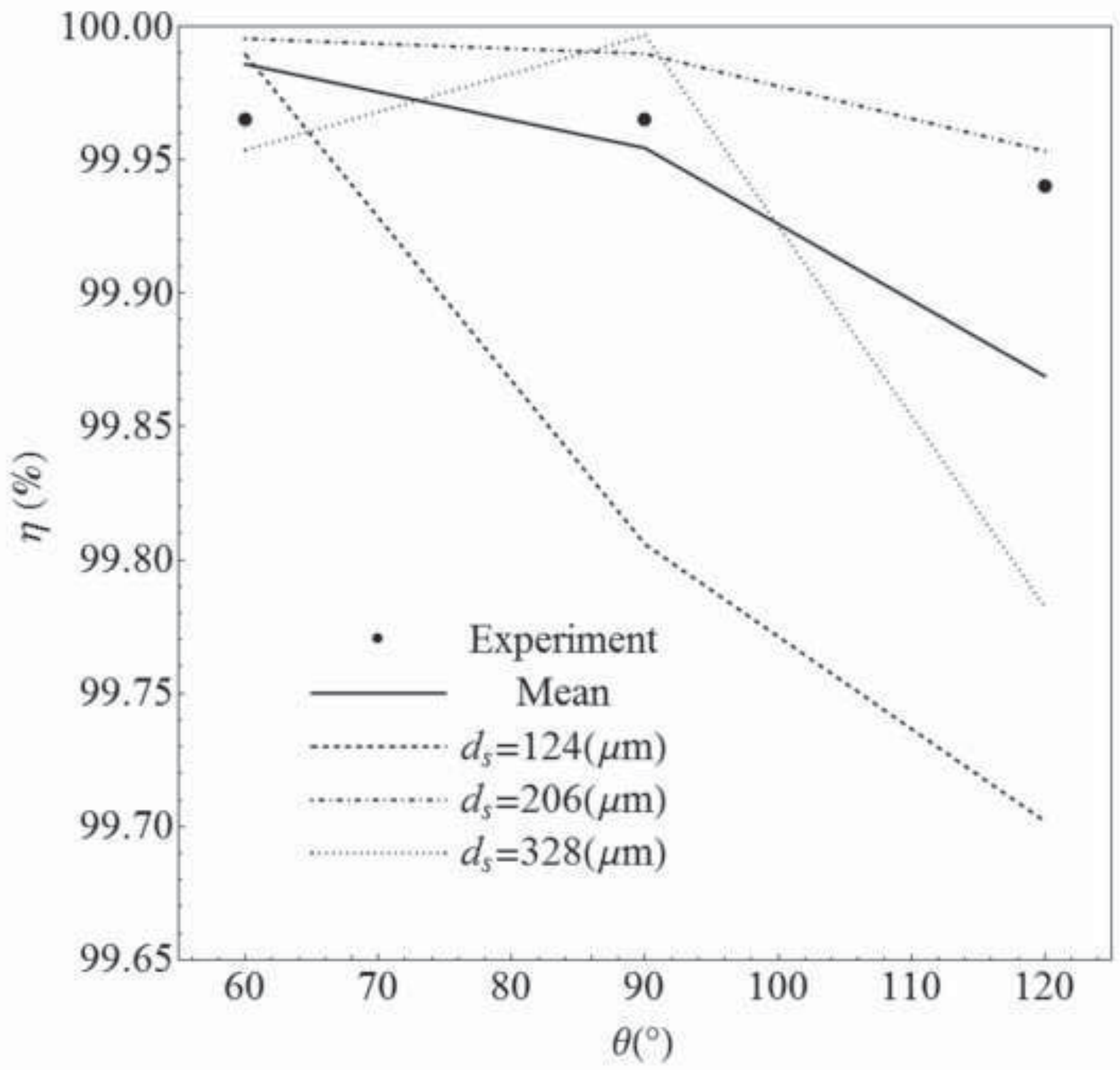



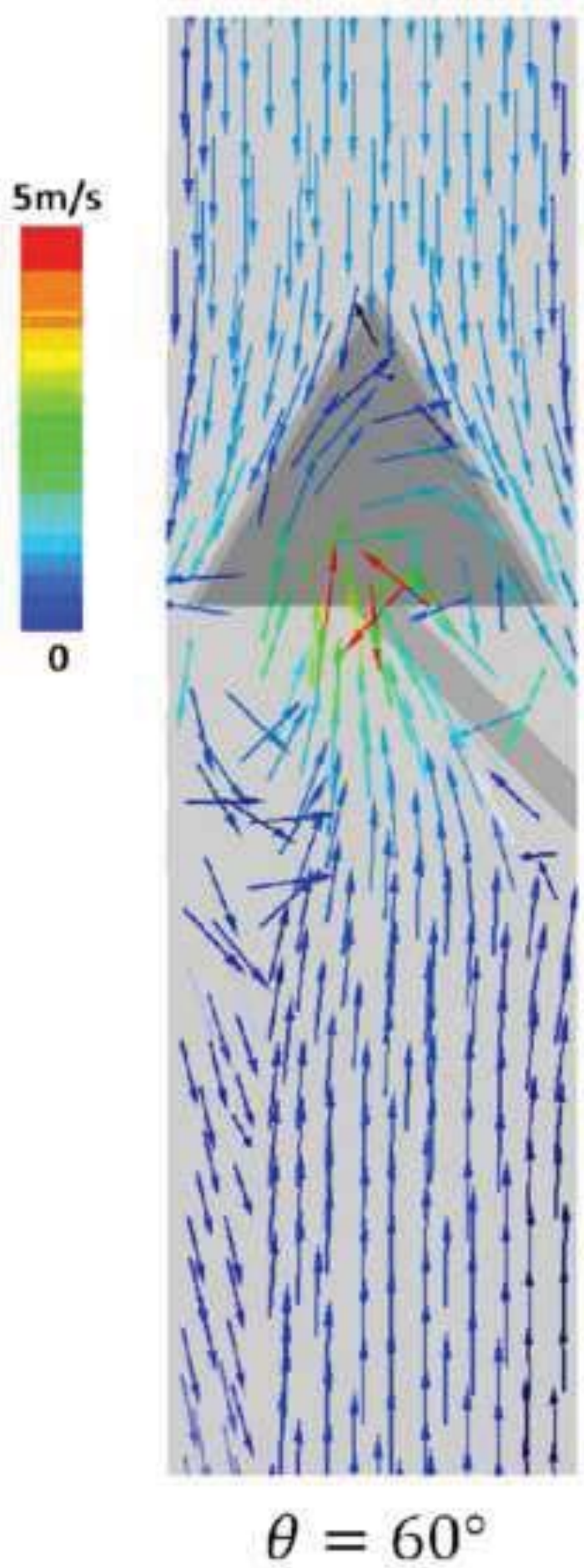
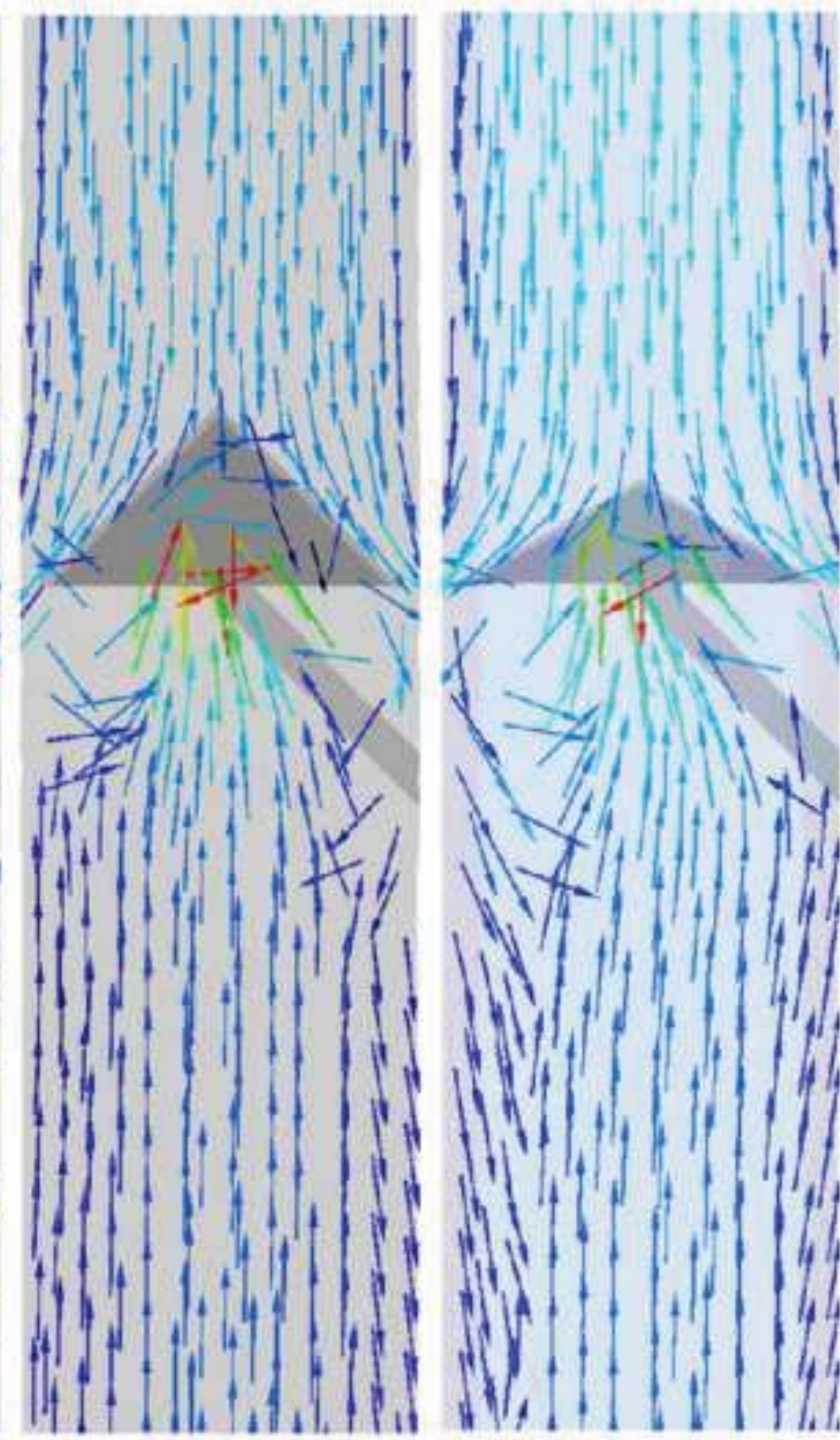

$$
\theta=90^{\circ}
$$
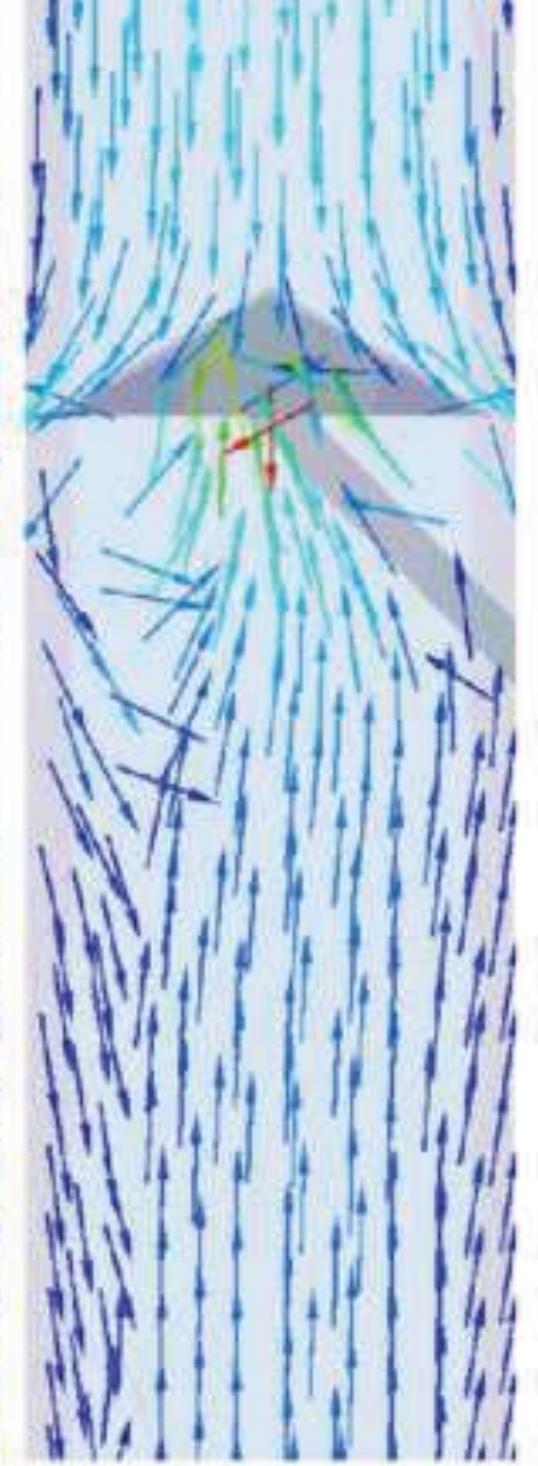

$\theta=120^{\circ}$

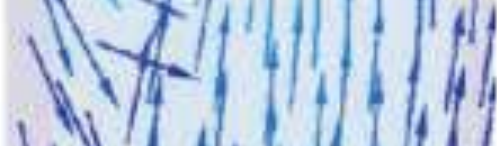



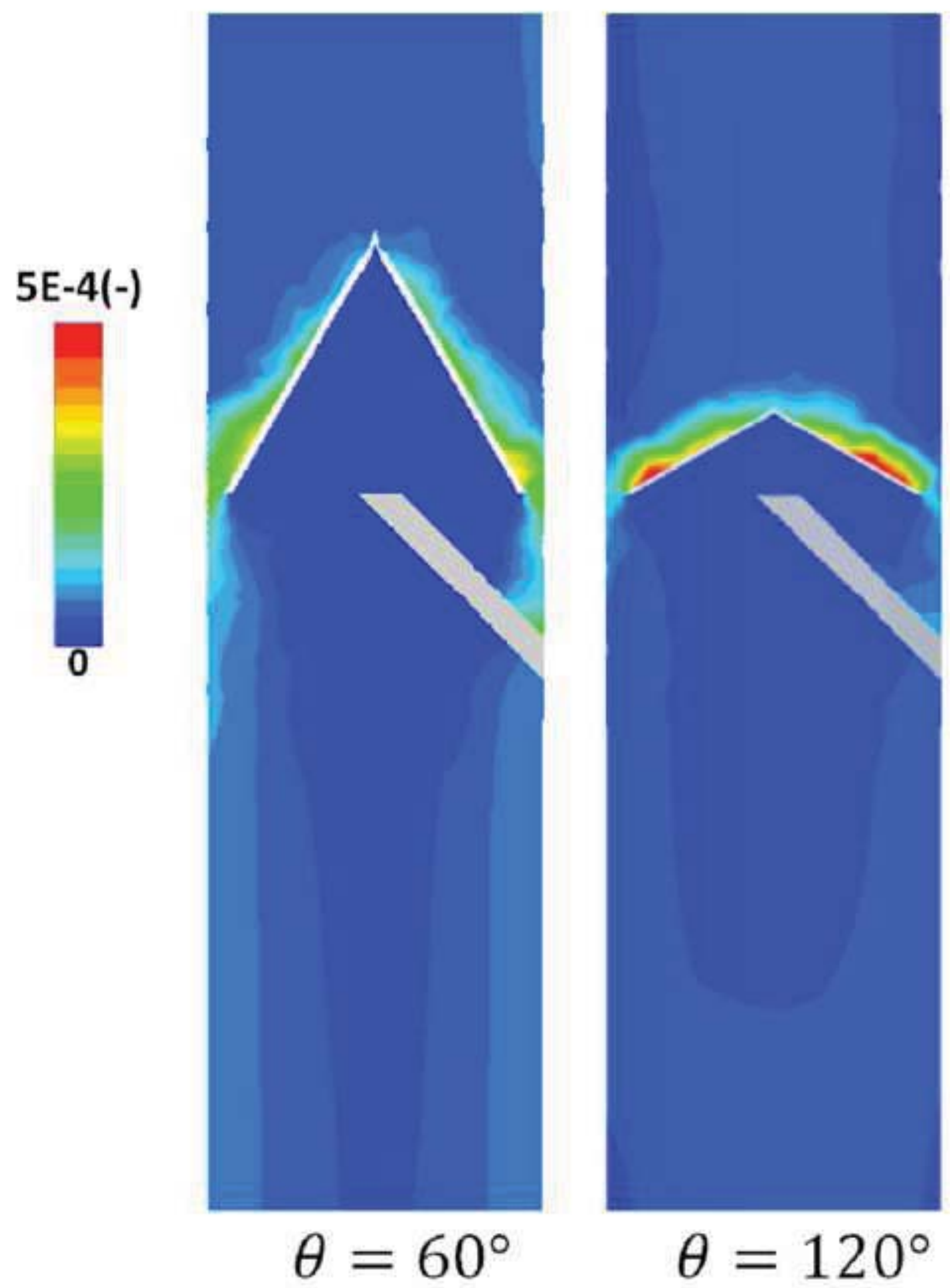






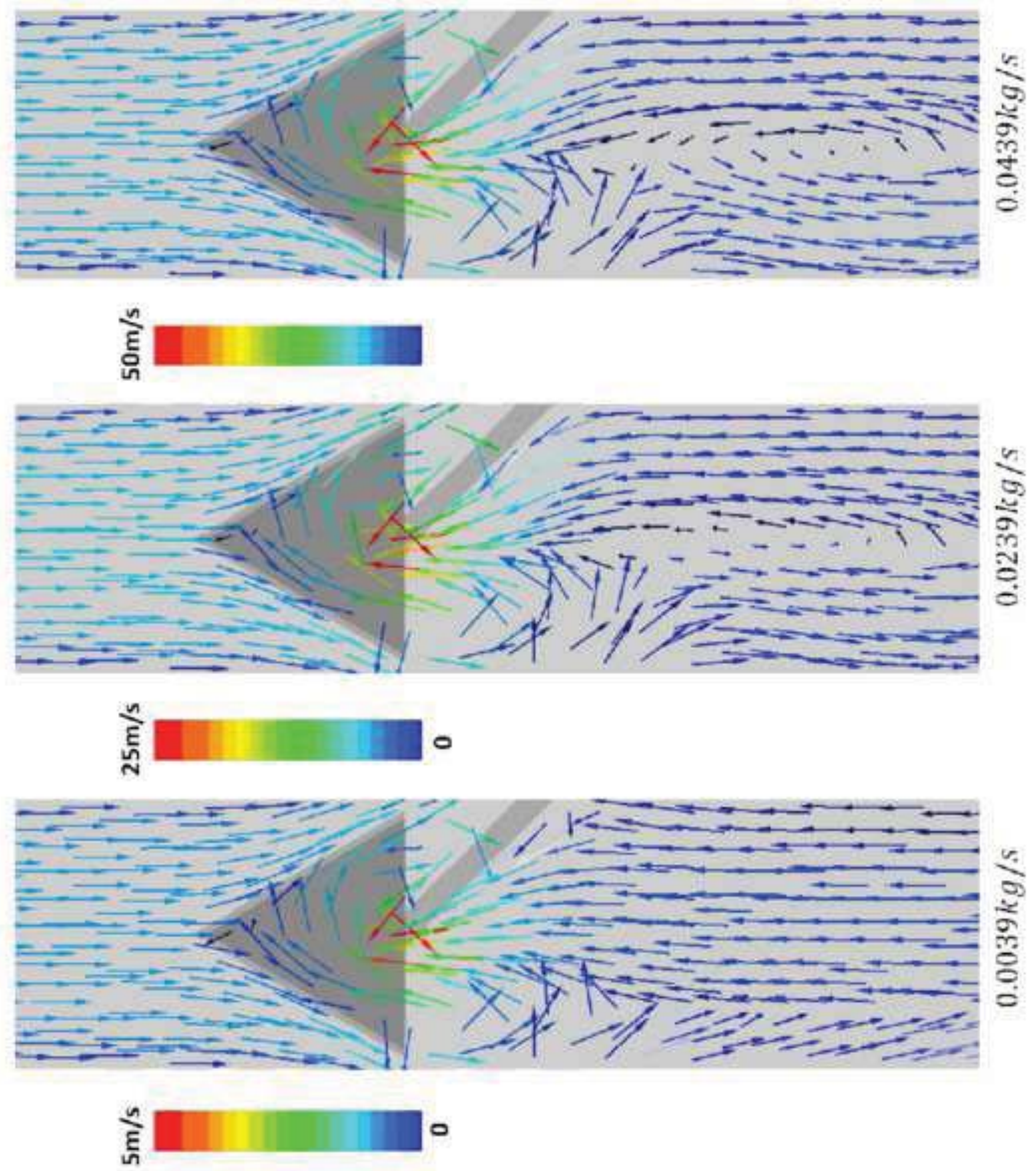
Click here to download high resolution image
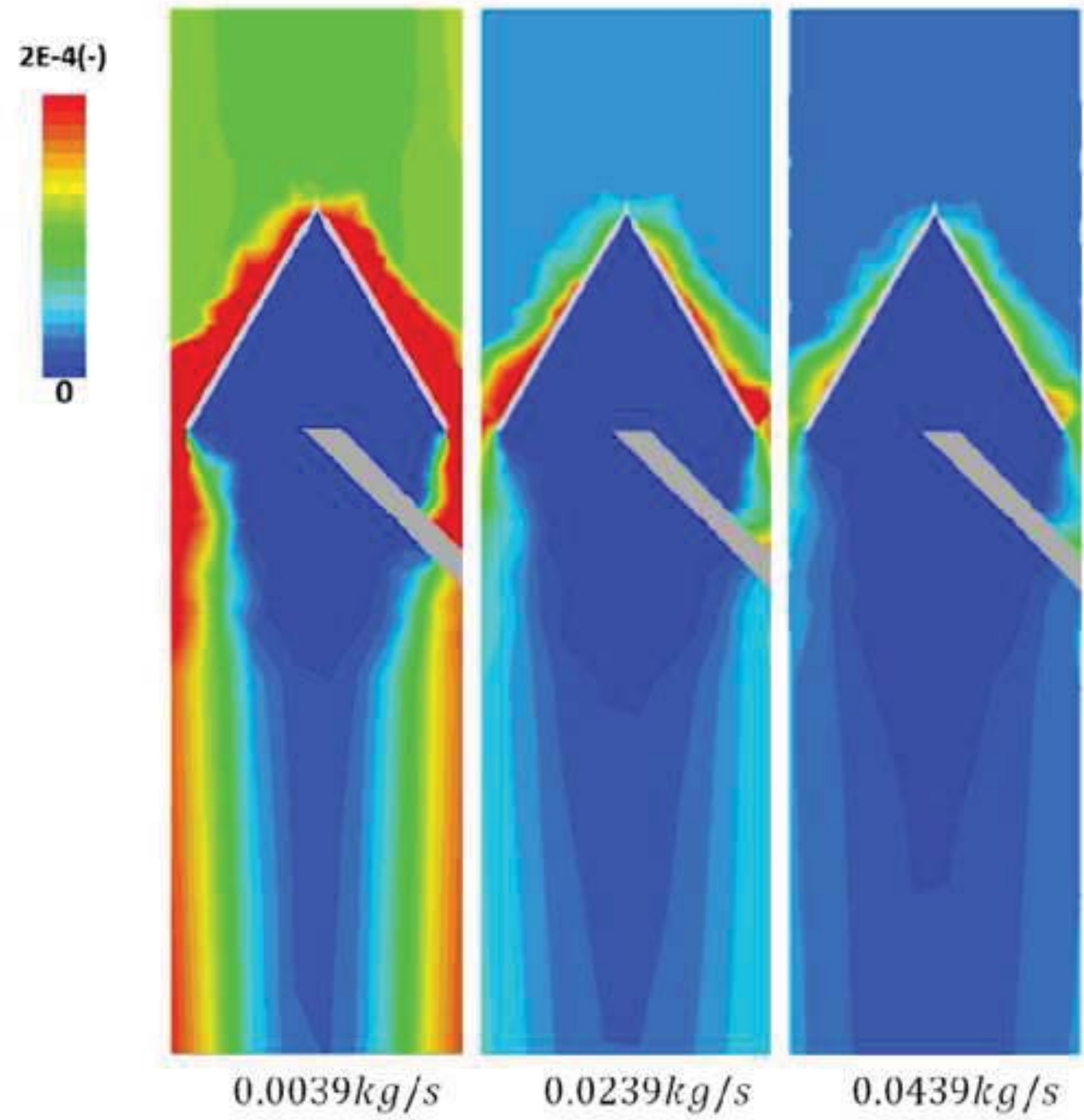

$0.0439 \mathrm{~kg} / \mathrm{s}$ 


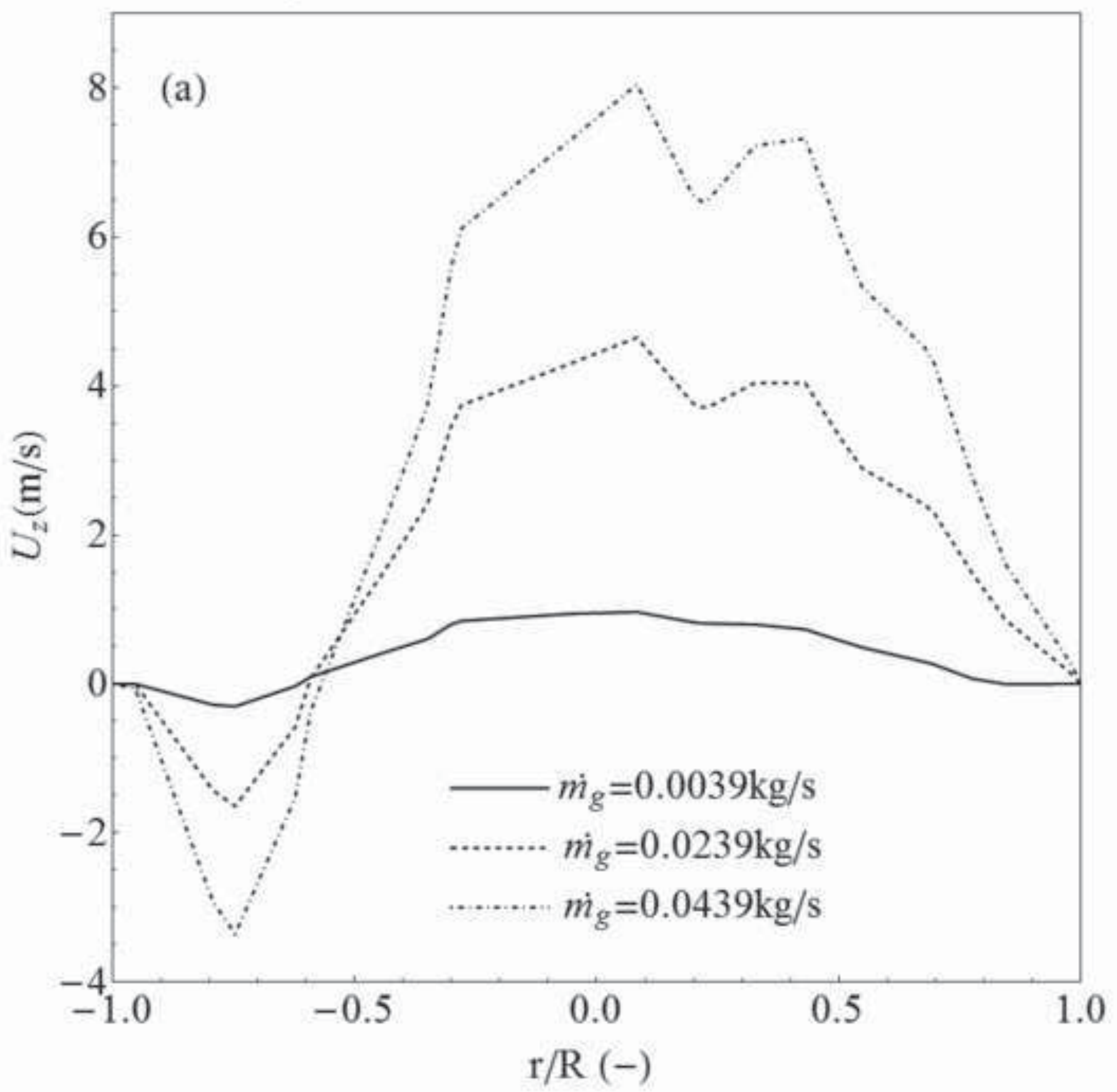









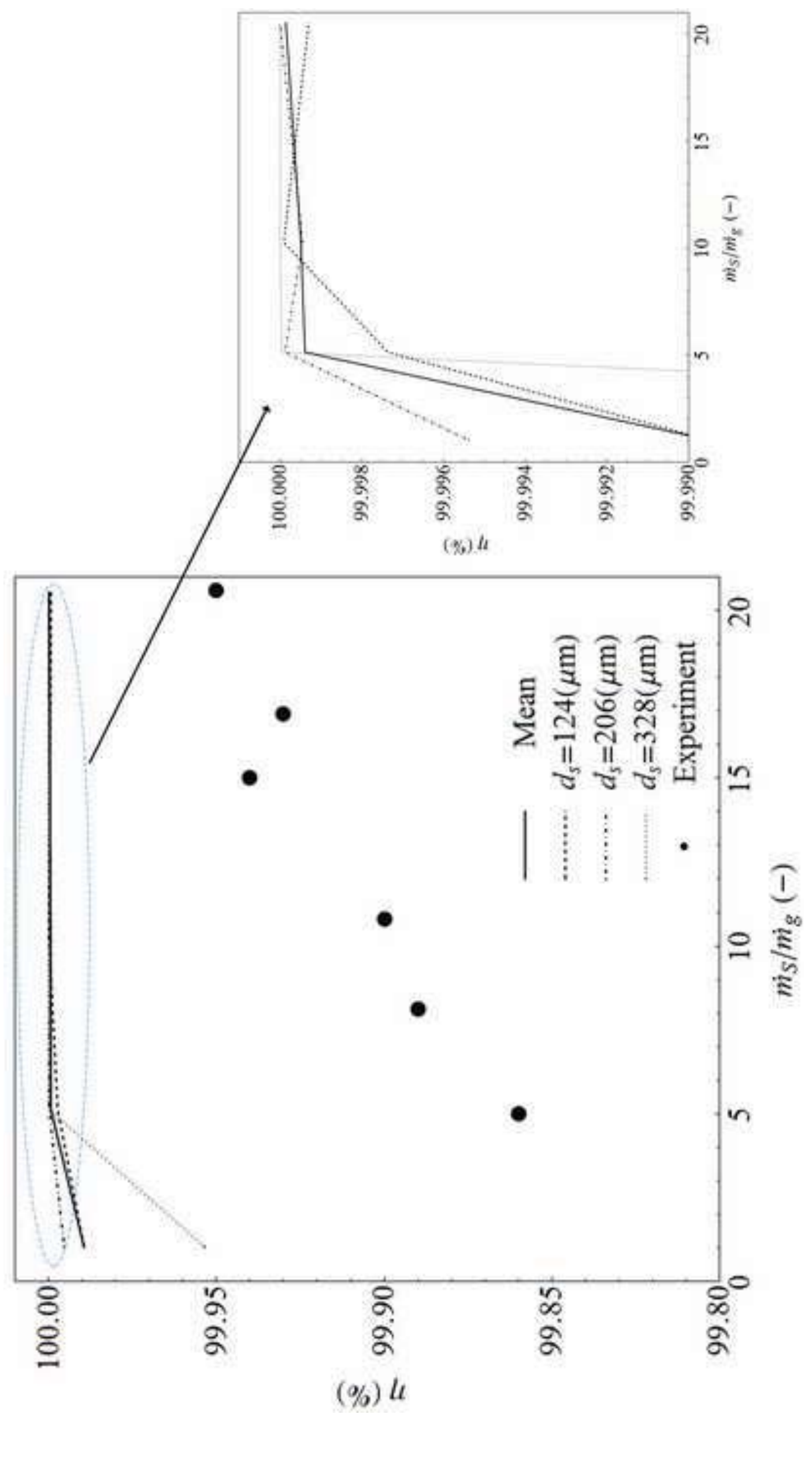





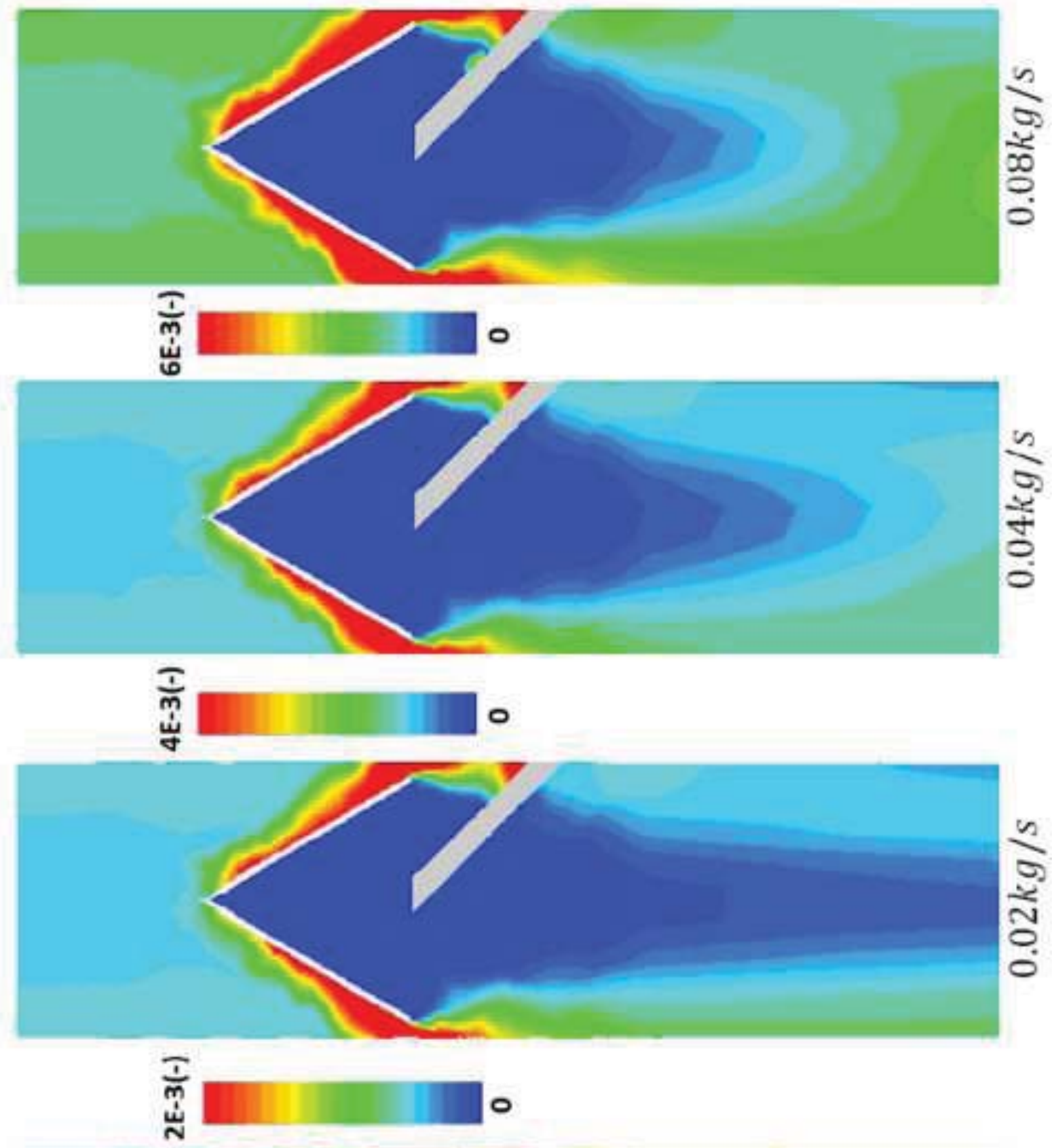

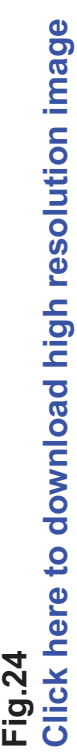
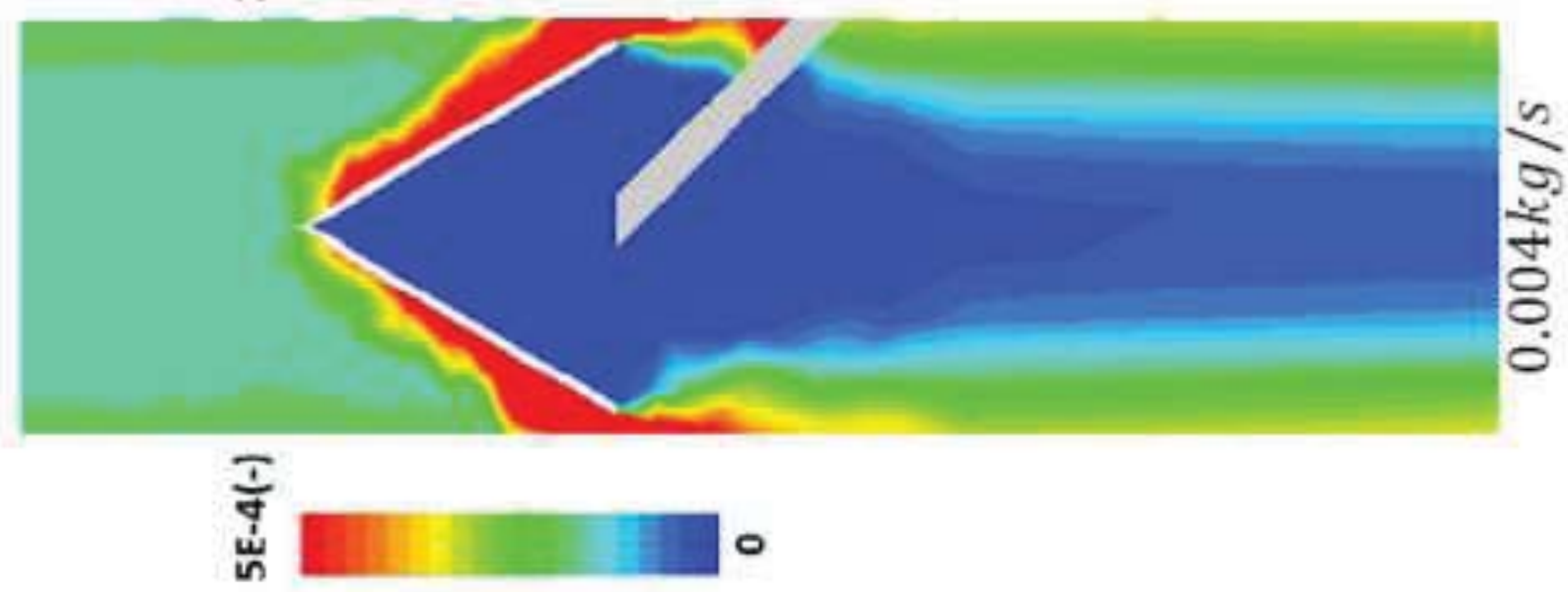


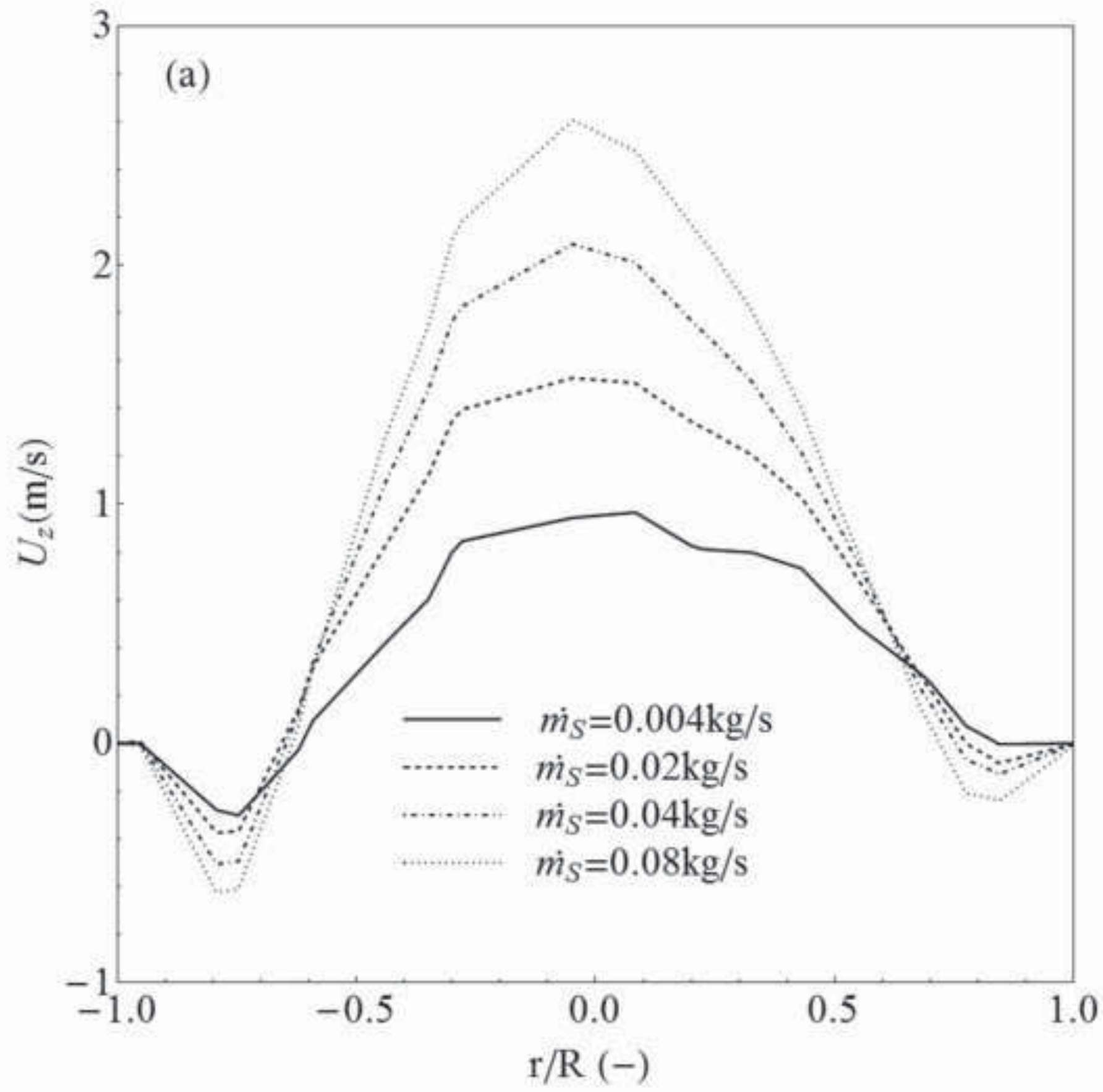




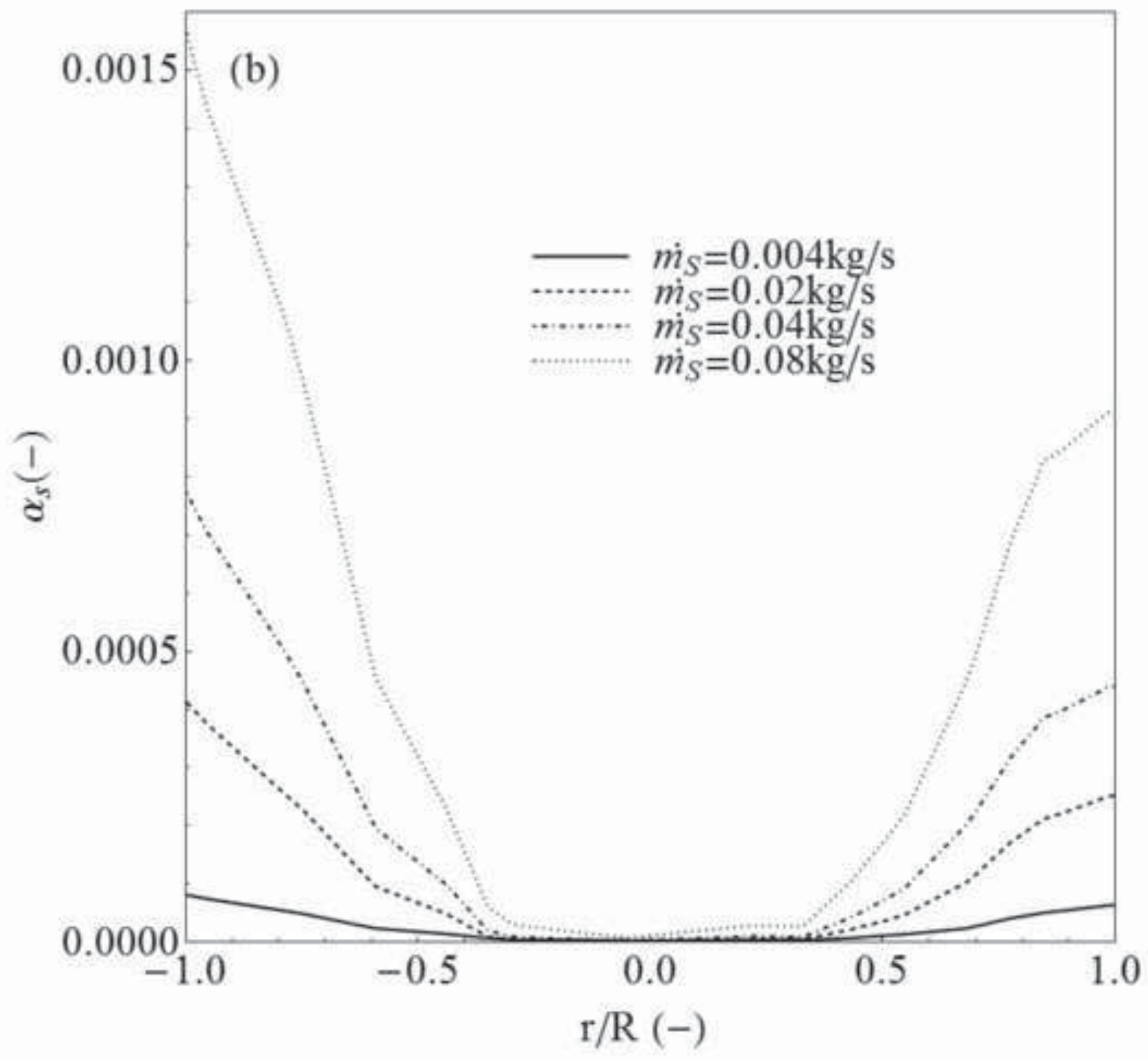




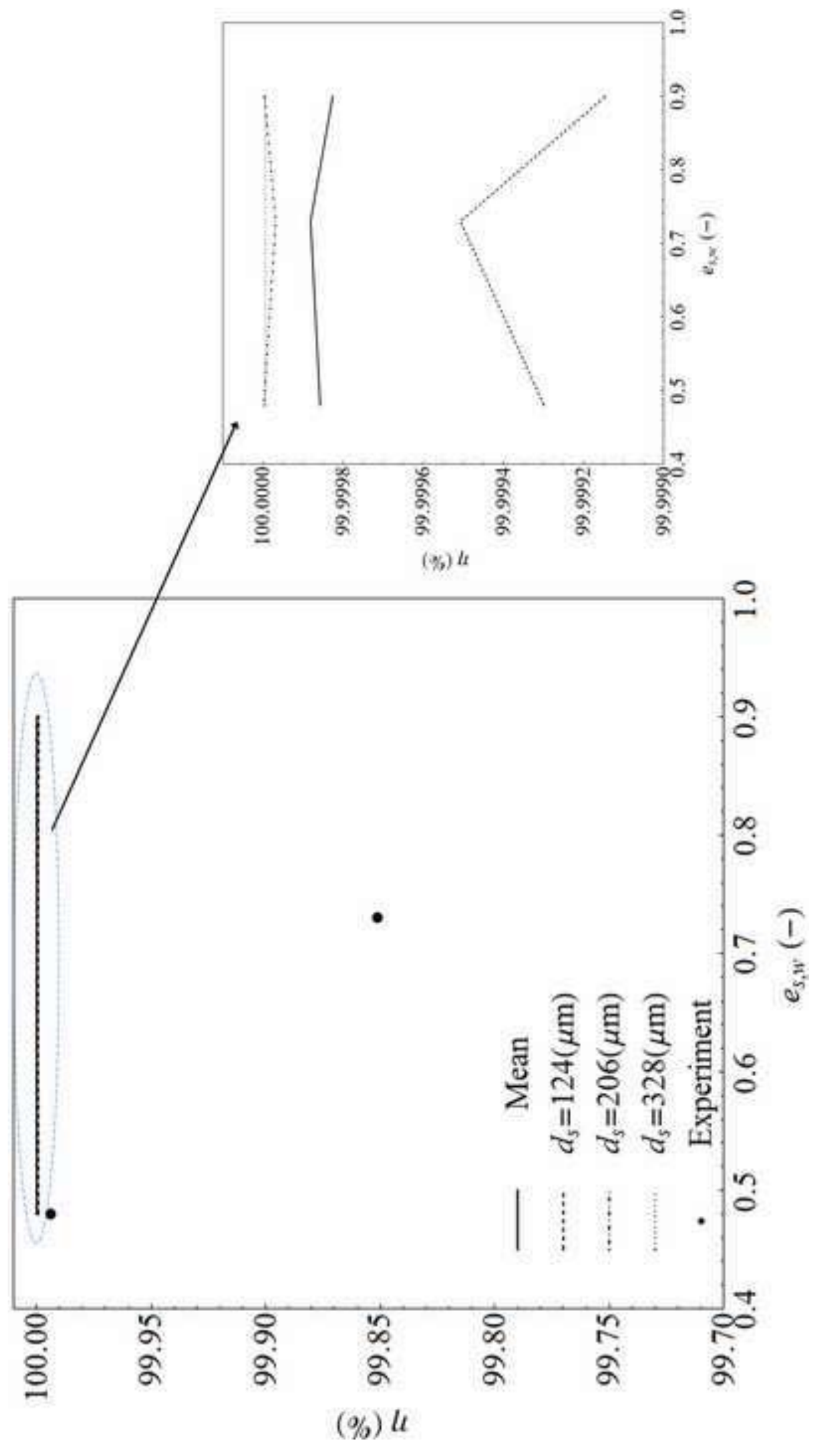

綂 


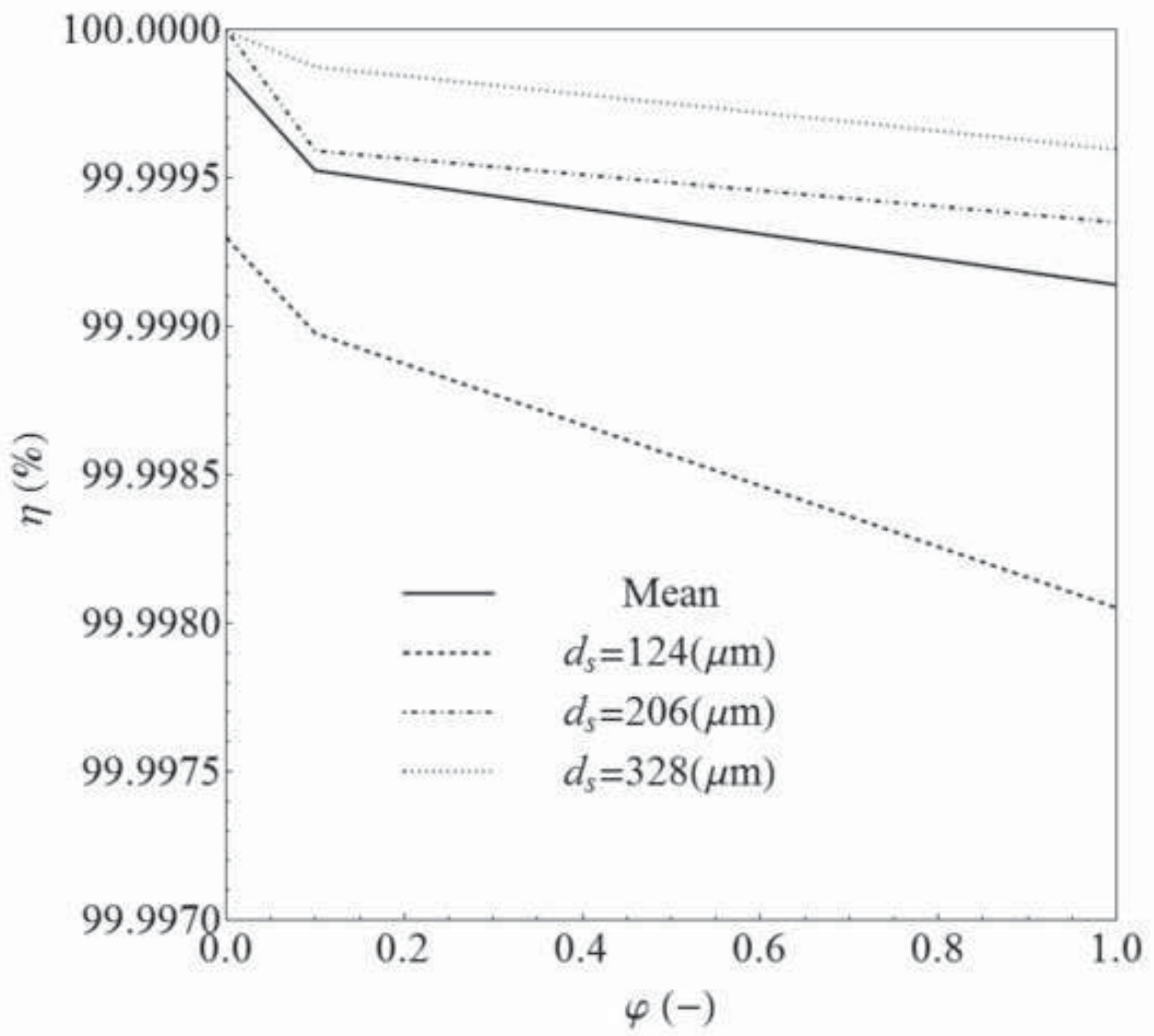




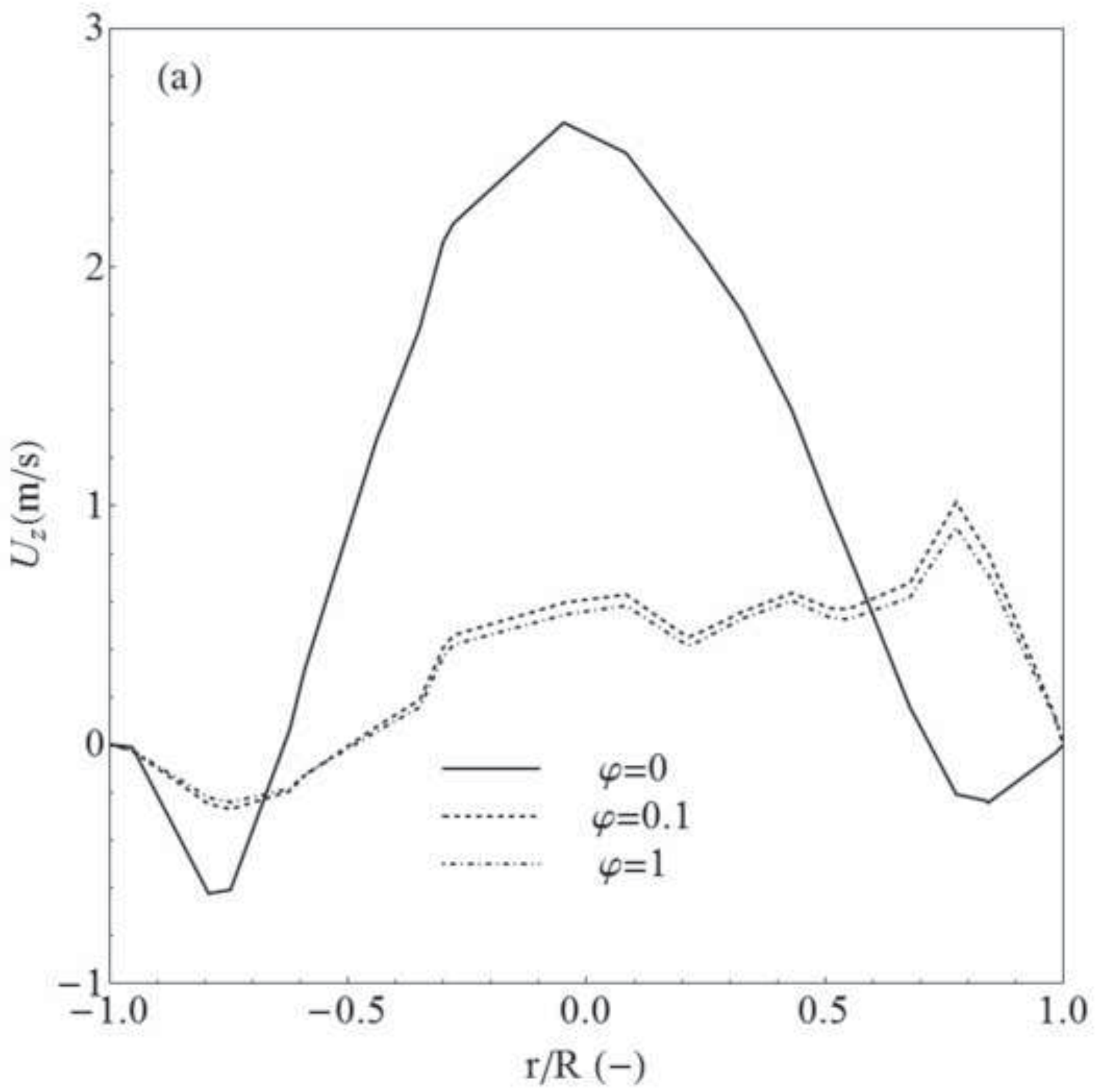

